\title{
Statistics of rare events in
} single-electron devices

Shilpi Singh

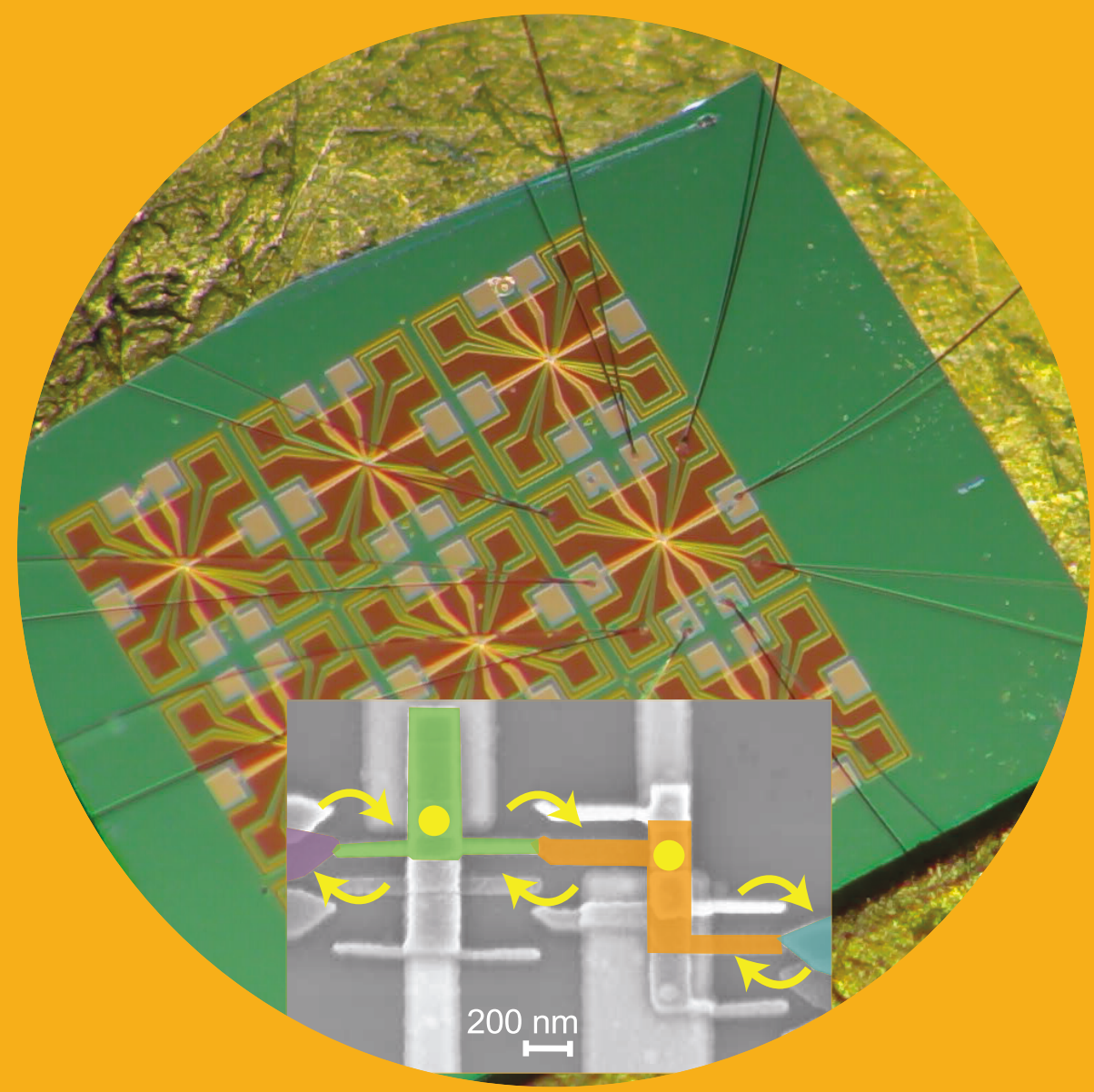

Aalto University 


\section{Statistics of rare events in single- electron devices}

Shilpi Singh

A doctoral dissertation completed for the degree of Doctor of Science (Technology) to be defended, with the permission of the Aalto University School of Science, at a public examination held at the lecture hall M1 (Otakaari 1, Espoo) of the school on 20th of February 2019 at 12. 
Professor Jukka P. Pekola, Aalto University, Finland

\section{Thesis advisors}

Dr. Dmitri S. Golubev, Aalto University, Finland

Dr. Joonas T. Peltonen, Aalto University, Finland

\section{Preliminary examiners}

Prof. Thomas Ihn, ETH Zurich, Switzerland

Dr. Ken Funo, RIKEN, Japan

\section{Opponent}

Prof. Sergio Ciliberto, Laboratoire de Physique de ENSL, France

DOCTORAL DISSERTATIONS 28/2019

(C) 2019 Shilpi Singh

ISBN 978-952-60-8419-0 (printed)

ISBN 978-952-60-8420-6 (pdf)

ISSN 1799-4934 (printed)

ISSN 1799-4942 (pdf)

http://urn.fi/URN:ISBN:978-952-60-8420-6

Unigrafia Oy

Helsinki 2019

Finland 
Author

Shilpi Singh

Name of the doctoral dissertation

Statistics of rare events in single-electron devices

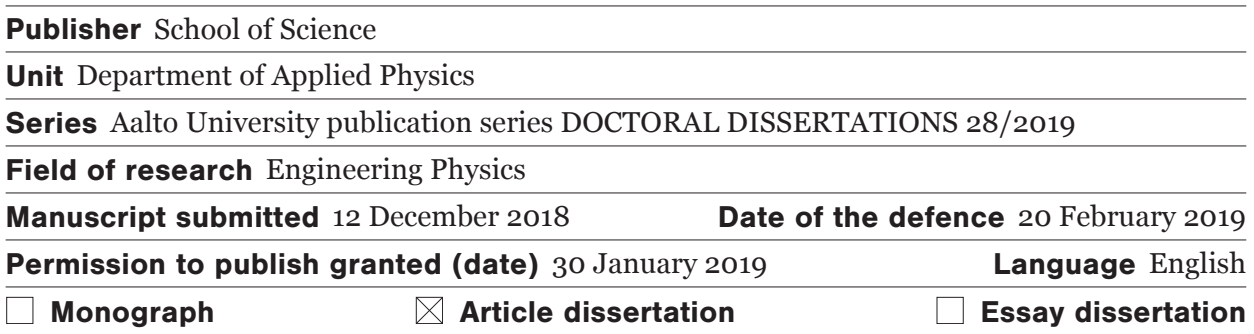

\section{Abstract}

Developments in fabrication and control of nanoscale devices have made precise single-electron counting possible. Due to the improved stability of these devices, increasing amounts of data can be collected leading to unprecedented statistics. These features have enabled the experimental verification of various statistical physics concepts, such as fluctuation relations and Maxwell's demon, with high precision.

The recent theory results on extreme fluctuations in the entropy produced by a system, and first passage times, have not yet been verified experimentally. The experimental studies of these theoretical concepts using single-electron devices are the focus of this thesis. The thesis starts with a brief introduction to the physics of single-electronic devices used in the experiments along with the experimental setup used to study them. Next, the experimental methods used to fabricate the samples and the basic sample characterization techniques are presented.

Later, the theoretical concepts are discussed and compared to the experimental results. This part starts with the probability distribution of the filtered telegraph signal from a bistable system, here a single-electron transistor. The filtering is done in two different ways: low pass filtering and finite time-averaging of the signal. The former allows us to propose a new method to obtain the transition rates between two states of the bistable system using the cumulants of its distribution. The latter allows us to see the rare fluctuations of current and observe theoretically predicted elliptic tail of the logarithm of the averaged current distribution.

Next, the stochastic entropy produced by a system is discussed. This part also includes the properties of its distribution and its minimum value. The theory is presented along with the experimental observations. Finally, an introduction to the theory of first-passage-time distributions is provided.

Keywords tunnel junction, single-electron devices, bistable system, stochastic thermodynamics, entropy, first passage times, fluctuation relations, large deviations, rare events

\begin{tabular}{lc}
\hline ISBN (printed) $978-952-60-8419-0$ & ISBN (pdf) $978-952-60-8420-6$ \\
\hline ISSN (printed) $1799-4934$ & ISSN (pdf) $1799-4942$ \\
\hline Location of publisher Helsinki & Location of printing Helsinki Year 2019 \\
\hline Pages 148 & urn http://urn.fi/URN:ISBN:978-952-60-8420-6 \\
\hline
\end{tabular}



To my family, plants and friends 


\section{Preface}

The work presented in this thesis was done in PICO group at Department of Applied Physics, Aalto University. The samples were fabricated in Micronova cleanroom facilities and for data analysis IT services provided by Aalto and Max Planck Institute for the Physics of Complex Systems (MPIPKS) were used.

First of all, I would like to thank my supervisor Prof. Jukka Pekola for accepting me as a $\mathrm{PhD}$ candidate, his patience and capability to survive my un-realistic timelines and connecting me to all the people who made this thesis possible.

Next most important part of my journey at Aalto has been my instructors, Dr. Dimtry (Dima) Golubev and Dr. Joonas Peltonen. I am grateful to Joonas for getting me started with experimental part of the projects, from cleanroom fabrication skills to measurement setups, and being there to answer all the questions related to the samples, tools and setups.

I thank Dima for helping me with the theory, introducing me to the art of cumulant generating functions, coffee company, and listening to all my rambles. It has been very nice to know you personally and I admire the simple way in which you formulate the answer to a complicated looking problem.

Along with my supervisor and instructors, I would like to thank my collaborators, Dr. Ivan Khaymovich, Dr. Èdgar Roldàn, Dr. Izaak Neri, Prof. Frank Jülicher, Prof. Keiji Saito, Prof. Ville F. Maisi, Dr. Jonne V. Koski, M. Sc. E. Mannila, Prof. Christian Flindt, and M. Sc. Paul Menczel. Ivan has been involved in almost all the projects I have presented in this thesis, which has an advantage that I can ask him any question related to theory part and I usually get some answer to it. Also, he is very prompt in replying to emails, chats and without him, the collaboration with Èdgar, Izaak, and Frank might not have occurred. I thank you Ivan for all these things.

Èdgar, Izaak, and Frank joined my list of collaborators in the later half of my $\mathrm{PhD}$. They have profoundly influenced the direction of my research. I thank you for the unforgettable conversations and introducing me to 
martingales, Doob's inequality, and all the other super powers.

I admire Èdgar's enthusiasm in research. It made me appreciate my data and results. Since, the start of our collaboration Èdgar has been a mentor for my research. I admire his patience in explaining the same thing again and again.

Izaak, I enjoy the mathematical preciseness that you strive towards. You restored my appreciation for mathematical detail and the purity that lies within. This might have affected some chapters of this thesis. I am sorry for the experimentalists that have to suffer the consequences.

I thank Frank for approving my visits to MPIPKS, providing me a platform to present our ongoing work and fruitful discussions.

I thank Keiji for initiating the project on first-passage times. This led to the double dot system that was used in $50 \%$ of the work presented here. I enjoyed our discussions together during your visit.

I thank Christian for introducing me to the presence of ellipses in a bistable system, and his capability to imagine a line segment as a small segment of an ellipse. I thank Paul for providing the numerical calculations for first passage in charge and useful discussions.

Before I forget, I would like to thank Jonne for getting me started with the physics of single-electron devices and Matlab, Ville for providing useful ideas on the implementation of feedback control in the double dot system, and Prof. Matthias Gramich for being a savior in the lab and an awesome co-main user of bonder.

I thank Dr. Juha Vartiainen for being my mentor in Aalto mentoring program, and for the nice experience with sailing.

Not to forget all the past and the present PICO group members and frequent visitors Dr. Hung Nguyen, Dr. Olli-Pentti Saira, Dr. Matthieu Taupin, Dr. Simone Gasparinetti, Dr. Timothé Faivre, Dr. Anna Feshchenko, Dr. Robab Najafi Jabdaraghi, Prof. ChiiDong Chen, Dr. Alberto Ronzani, M. Sc. Antti Jokiluoma, M.Sc. Antti Moisio, M.Sc. Klaara Viisanen, M.Sc. Jorden Senior, M.Sc. Libin Wang, M. Sc. Ilmo Räisänen, M. Sc. Randy Chang, Dr. Michele Campisi, Dr. Maciej Zgirski, Dr. Shuji Nakamura, Dr. Bivas Dutta, M. Sc. Brecht Donvil, M.Sc. Bayan Karimi, B. Sc. Jesse Muhojoki, Dr. Azat Gubaydullin, Dr. Olivier Maillet, Dr. George Thomas, M. Sc. Rishabh Upadhyay, and M.Sc. Diego A. Subero for providing help and support in addition to the pleasant working environment.

I thank Dr. Minna Gunes for keeping the administrative work going and always ready to provide a helping hand.

I thank M.Sc. Matti Partanen, Klaara, Somendu, and Dibyendu for being awesome friends. I appreciate their patience in listening to my never lasting stories, waiting for me even though it might seem sometime forever, being my yoga buddy and being a part of mid-night badminton team.

I thank you Tiziana and Tuomas for being awesome friends and keeping in touch although we are miles apart. I thank Eija for a lot of hugs, Milicia 
for board games, Michiko and Vaibhav for lunch company, Prof. Tapio A. Nissila and MSP group for awesome summer parties that I was allowed to crash into. I also thank D. Sc. Ivan Degtyarenko for helping with triton and all the other former and current members of the 4th floor of the old main building.

I thank my professors from home, Prof. R. Nagarajan, Prof. Vijay A. Singh, and Prof. Sandip Ghosh for introducing me to research and for being a constant source of inspiration. My friends from college days, it is nice of you to be still in touch and keep my spirits up when I feel low.

For Navneeta and Sri, it's quite hard for you to get rid of me :) Thank you for being there to receive my mid-night calls and just listening to what I want to say and being able to keep up with me. I thank my plants for providing me company throughout my $\mathrm{PhD}$ life.

I thank my grandparents, parents, and brothers for their continuous support and adding meaning to the life. You are awesome and irreplaceable! Maathru Devo Bhava! Pithru Devo Bhava!

Samu, mun kulta, kiitos kaikesta! not that I am letting you go now :) Kiitos mahtavista seikkailuista, kaikista auringon nousuita ja laskuista keskiyön pyöräretkillä. Kiitos ihanasta seurasta ja ruoasta. A lots of hug for you and your family for making Finland a home far from motherland.

I am pretty sure I might have missed some of you. I would like you to know that it was unintentional and due to lack of my memory. Thank you for your help! Great! You are an achiever, you made it so far and are still reading :)

Espoo, January 31, 2019,

Shilpi Singh 
Preface 


\section{Contents}

$\begin{array}{ll}\text { Preface } & \text { iii }\end{array}$

Contents $\quad$ vii

$\begin{array}{ll}\text { List of Publications } & \text { ix }\end{array}$

Author's Contribution $\quad$ xi

1. Introduction $\quad 1$

2. Physics of single-electron devices 5

2.1 Tunnel junction . . . . . . . . . . . . . . 5

2.2 Single-electron box . . . . . . . . . . . . . 8

2.3 Single-electron transistor . . . . . . . . . . . . 10

2.3.1 Measurement protocol . . . . . . . . . 13

2.4 Electronic double dot system . . . . . . . . . . . . . 14

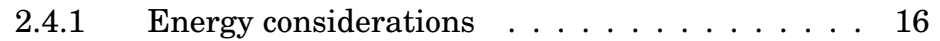

2.4 .2 Transition rates . . . . . . . . . . . 18

2.4.3 Current through the double dot . . . . . . 19

2.4.4 Measurement protocol ........... 20

3. Experimental methods 23

3.1 Sample fabrication . . . . . . . . . . . . . . 23

3.2 Device characterization . . . . . . . . . . . . 27

3.2.1 Single-electron transistor . . . . . . . . . . 27

3.2.2 Double-island structure . . . . . . . . . 28

3.3 Measurement setup . . . . . . . . . . . . . 30

4. Current distribution of a bistable system 33

4.1 Output current . . . . . . . . . . . . . . 33

4.2 Calculation of the transition rates using low pass filtering 35

4.2.1 Probability distribution . . . . . . . . . . 35

4.2 .2 Transition rates . . . . . . . . . . . 37 
4.2.3 Experimental test of the cumulant method . . 38

4.3 Distribution of time-averaged currents . . . . . . . 38

4.3.1 Steady-state probability of states . . . . . . 39

4.3 .2 Probability distribution . . . . . . . . . . . 40

4.3 .3 Short-time limit . . . . . . . . . . . . . 41

4.3.4 Long-time limit . . . . . . . . . . . . . 43

5. Stochastic thermodynamics of an electronic double dot 47

5.1 Master equation . . . . . . . . . . . . 47

5.2 Stochastic entropy production . . . . . . . . . 48

5.2.1 Stochastic entropy production in an electronic double dot . . . . . . . . . . . . 50

5.3 Distribution of entropy $\ldots \ldots \ldots \ldots \ldots$

5.4 Extreme values of entropy $\ldots \ldots \ldots \ldots \ldots$

6. First-passage times and extreme fluctuations 57

6.1 Introduction . . . . . . . . . . . . 57

6.1.1 Charge distribution $P(t, N)$ : its evolution equation and properties ............. 58

6.1 .2 Survival probability . . . . . . . . . 58

6.1.3 First-passage-time distribution . . . . . . 60

6.1.4 Distribution of extreme-values . . . . . . . . . 60

6.2 Charge transfer through a tunnel junction . . . . . . . . 61

6.3 Approximation for a double dot system . . . . . . . . 63

$\begin{array}{ll}\text { 7. Summary } & 69\end{array}$

$\begin{array}{ll}\text { References } & 73\end{array}$

$\begin{array}{ll}\text { Publications } & 81\end{array}$ 


\section{List of Publications}

This thesis consists of an overview and of the following publications which are referred to in the text by their Roman numerals.

I S. Singh, E. Mannila, D. S. Golubev, J. T. Peltonen, and J. P. Pekola. Determining the parameters of a random telegraph signal by digital low pass filtering. Applied Physics Letters, Volume 112, Issue 243101 (5 pages), June 2018.

II S. Singh, J. T. Peltonen, I. M. Khaymovich, J. V. Koski, C. Flindt, and J. P. Pekola. Distribution of current fluctuations in a bistable conductor. Physical Review B, Volume 94, Issue 24, 241407 (5 pages), December 2016.

III S. Singh, É. Roldán, I. Neri, I. Khaymovich, D. S. Golubev, V. F. Maisi, J. T. Peltonen, F. Jülicher, and J. P. Pekola. Extreme reductions of entropy in an electronic double dot. arXiv:1712.01693, (16 pages), Submitted 2018.

IV S. Singh, P. Menczel, D. S. Golubev, I. M. Khaymovich, J. T. Peltonen, C. Flindt, K. Saito, É. Roldán, and J. P. Pekola. Universal First-PassageTime Distribution of Non-Gaussian Currents. arXiv:1809.06870, (10 pages), Submitted 2018. 
List of Publications

X 


\section{Author's Contribution}

Publication I: "Determining the parameters of a random telegraph signal by digital low pass filtering"

The author fabricated the sample A, performed the experiment on it, and wrote the first version of the manuscript.

\section{Publication II: "Distribution of current fluctuations in a bistable conductor"}

The author fabricated the sample, performed the experiment, analyzed the data, and wrote the experimental part of the manuscript.

Publication III: "Extreme reductions of entropy in an electronic double dot"

The author fabricated the sample, performed the experiment, analyzed the data, and participated in conceiving and designing the experiment and writing of the article.

\section{Publication IV: “Universal First-Passage-Time Distribution of Non-Gaussian Currents"}

The author fabricated the sample, performed the experiment, analyzed the data, and participated in conceiving and designing the experiment and wrote the first version of the manuscript. 
Author's Contribution

xii 


\section{Introduction}

The improbable is bound to happen one day.

Statistics of Extremes

E. J. Gumbel

A macroscopic system is often approximated as a huge collection of independent subsystems. For such a system, averages are usually enough to describe its physical properties. When the system size decreases, the relative fluctuations become more and more prominent. These fluctuations are often approximately described by a Gaussian distribution [1], in accordance with the central limit theorem [1-4]. However, the tails of the distribution, describing the rare events, do not strictly follow the Gaussian form. This departure is governed by the large deviation principle [5].

Although the rare events are seldom, their consequences can be tremendous and therefore they have been studied in various context, such as financial crises [6-8] and natural disasters [9, 10]. In electronics, the study of rare events is important, for example, it can be used to determine the accuracy of the devices. One such application is the calculation of probability of dark counts in the threshold single photon detectors [11, 12]. However, the sporadic nature of rare events makes it difficult to gather sufficient amount of data to carry out the statistical analysis. Fortunately, the single-electron devices provide an excellent test bench [13] for studies of rare events as they enable one to collect a huge amount of data and reliably analyze the tails of the distribution for various fluctuating quantities, such as current and entropy.

The rare events do not usually have a significant contribution on the average of the distribution $\langle x\rangle$. However, it is possible that the average of a function $\langle f(x)\rangle$ is strongly influenced by these rare events. In statistical physics, this is illustrated by the well known integral fluctuation relations (IFRs) [14-17] derived for quantities such as work [16, 18] and entropy production [17]. In the case of entropy production $\Delta S$, the integral fluctuation relation states $\left\langle e^{-\Delta S}\right\rangle=1$, where the strong but few negative 
fluctuations of $\Delta S$ have a significant impact on the average value of the exponent $e^{-\Delta S}$. A sufficient condition for IFR is usually the corresponding detailed fluctuation relation [17-26]. The fluctuation relations have been confirmed in different experimental setups [27-31], including electronic systems [32-34].

The average in the integral fluctuation relation is taken over all the possible realizations. These realizations are usually stochastic in nature, i.e., they fluctuate in time and from one realization to another. This gives the field its name stochastic thermodynamics [35-37] when one investigates the thermodynamic quantities, such as entropy production, work and heat, at the level of individual trajectories.

For these quantities, theoretical bounds have been recently obtained, quantifying the statistics of their extreme values during the time evolution. One such study is done in Ref. [38] where a bound is derived for the statistics of the minimum entropy production of a realization.

Another way to quantify the rare events is to investigate the time taken by a stochastic trajectory to hit a given value for the first time, known as the first-passage time [39, 40]. The study of first-passage times has a long history starting from early 20 th century [41, 42] and spans over different fields, such as Brownian motion [39-44], biochemistry [45-50], astrophysics [51, 52], decision theory [53-55], searching problems [56, 57], and finance $[8,58]$. Lately, they have also been applied to stochastic thermodynamics [38, 55, 59-63]. However, these first-passage times and the extreme fluctuations in the entropy production have not yet been experimentally verified in an electronic setup.

In this thesis, we experimentally study these theoretical concepts using single-electron circuits $[64,65]$. These devices are made of metallic tunnel junctions which were initially proposed in Refs. [66-71] and first realized in Ref. [72]. The tunnel junction circuits are governed by the physics of quantum tunneling and classical electrostatics. The thesis focuses on experiments on single-electron transistors (SETs) and electronic double dots consisting of two and three tunnel junctions connected in series. Here and further, we use 'double dot' to refer to a device with two metallic islands with a dimension of few $100 \mathrm{~nm}$ to $1 \mu \mathrm{m}$. The energy level spacing in such islands is very small $(\approx \mathrm{neV})$ compared to the other energy scales in the device. In Chapter 2, we discuss the physics of these single-electron devices in detail.

The devices used in this thesis are fabricated using the Fulton-Dolan method [72-74]. Chapter 3 provides the fabrication method used to process the samples and a brief discussion of the methods used to characterize them, ending with a short note on the measurement setup.

Chapter 4 focuses on the study of the current output through an SET coupled to the island of another SET. The measurements are done at low temperatures and low bias voltages where the system is in the Coulomb 
blockade regime, and the SET acts as a bistable system. We filter the output currents using two different filtering functions, namely low-pass filtering (Sec. 4.2) and time averaging (Sec. 4.3), and compute their probability distributions. The low-pass filtering mimics the behavior of a slow detector. We use the cumulants of low-pass filtered currents to compute the transition rates between the system states. This method works even in the case of a slow detector. Section 4.2 summarizes the results of Publication I.

In Sec. 4.3, we present the distribution of time-averaged current, which can be used to study the current fluctuations through an electronic system when coupled to a bistable fluctuator. The distribution demonstrates the deviation of averages of fluctuations from the central limit theorem in the long averaging-time limit, where the departure given by the large deviation principle is evident. We observe the tail of the distribution due to rare events and show the elliptic nature of the logarithm of the distribution. We also observe a fluctuation relation for the departure of the time-averaged current from its mean value. This section summarizes the findings of Publication II.

Chapter 5 focuses on the statistics of the minimum stochastic entropy production in an electronic double dot in nonequilibrium. Our experiment essentially verifies the theoretical findings of Ref. [38] and emphasizes the condition of steady state for the results to hold. The chapter starts with a brief introduction to a master equation, governing the dynamics of a Markovian system. Next, the definition of entropy production and the corresponding fluctuation relations are provided. The chapter ends with the extreme value statistics [9] of the entropy produced in the system including the bounds on total entropy and heat exchange with environment. This chapter presents the results of Publication III.

Chapter 6 focuses on the first-passage times for a fixed number of electrons to be transferred through the double dot. The chapter starts with a general introduction to the first-passage times and probabilities of extreme values for a stochastic process, see Sec. 6.1. Subsequently, the first-passage times for the transport of electrons through a tunnel junction are computed, see Sec. 6.2. This simple model is then used to study the more complicated dynamics of an electronic double dot, see Sec. 6.3. This chapter provides the theoretical framework and experimental results of Publication IV. Chapter 7 concludes the thesis with a brief note on possible future directions of research on this subject.

This thesis can be read in the following manner: those who are interested in the experimental part of the thesis, should start with Chapter 2 to get an idea about the devices studied in this thesis and then read Chapter 3 for details on fabrication and sample characterization techniques. Those who are more interested in understanding the physical quantities studied in this thesis, without getting into the experimental details, may skip Chapter 3. This thesis summarizes the findings of Publications I-IV, where 
Introduction

the additional details can be found. 


\section{Physics of single-electron devices}

This thesis presents the results of experiments done on single-electron systems. Before going into the details of the investigated physical quantities, we will discuss the various electronic devices studied in this thesis.

This chapter starts with a brief introduction to tunnel junctions that act as the building blocks of single-electron devices. Next, we present the single-electron transistor (SET) and an electronic double dot. These are the devices on which the experiments presented in this thesis have been performed. The brief overview of the experimental methods, such as fabrication details and basic characterization techniques, is presented in Chapter 3.

\subsection{Tunnel junction}

A tunnel junction is made up of two metallic layers with a thin insulator between them. In this work, based on the metals used, these two layers can be either normal metallic (N) or superconducting (S). The samples studied are made of aluminum and copper as the metallic layers. A 2-3 nm thin aluminum oxide $\left(\mathrm{Al}_{2} \mathrm{O}_{3}\right)$ layer is used as an insulator (I) between them forming a NIS junction at temperatures below $1.2 \mathrm{~K}$, i.e., the critical temperature $T_{\mathrm{c}}$ of bulk $\mathrm{Al}$ [75]. The scanning electron micrograph in Fig. 2.1 (a) shows one such tunnel junction with the $\mathrm{Cu}$ layer marked in green and the $\mathrm{Al}$ layer in turquoise. The side view and a simplified sketch of the junction are shown below the micrograph.

The Hamiltonian of a system with a tunnel junction connecting two electrodes 'left' $(L)$ and 'right' $(R)$ can be written as [64]

$$
\hat{H}=\hat{H}_{L}+\hat{H}_{R}+\hat{H}_{T} .
$$

The $\hat{H}_{L}$ and $\hat{H}_{R}$ are the Hamiltonians of the electrodes. For example, for normal-metal electrodes, they are given by $\hat{H}_{L}=\sum_{l} \epsilon_{l} \hat{a}_{l}^{\dagger} \hat{a}_{l}$ and $\hat{H}_{R}=$ $\sum_{r} \epsilon_{r} \hat{c}_{r}^{\dagger} \hat{c}_{r}$ where $\hat{a}_{l}\left(\hat{c}_{r}\right)$ and $\hat{a}_{l}^{\dagger}\left(\hat{c}_{r}^{\dagger}\right)$ are the annihilation and creation operators associated with energy $\epsilon_{l}\left(\epsilon_{r}\right)$ of the conduction electron reservoir in 
(a)

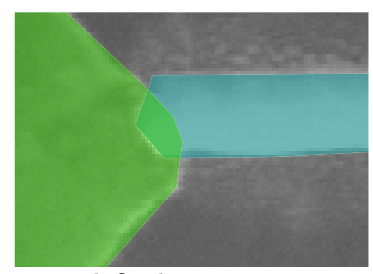

simplified side view sketch

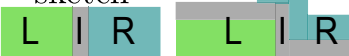

(b)

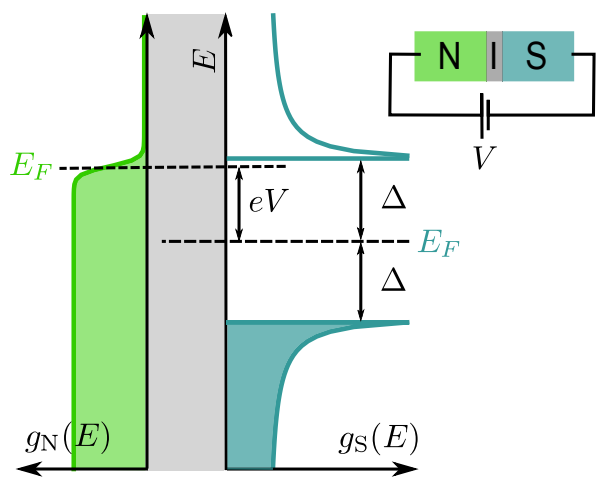

Figure 2.1. (a) Top: Scanning electron micrograph (SEM) of a tunnel junction connecting left $(\mathrm{L})$ and right $(\mathrm{R})$ electrodes. Bottom: A simplified sketch of the tunnel junction (left) and its side view (right). (b) The energy diagram of the junction with $\mathrm{L}$ as a normal metal $(\mathrm{N})$ and $\mathrm{R}$ as a superconductor $(\mathrm{S})$. The electrodes are kept at a potential difference of $V$ leading to a difference of $\mathrm{eV}$ in the Fermi energies. The shaded region shows the function $g_{i}(E)=n_{i}(E) f_{i}(E)$, a product of density of states $n_{i}(E)$ and Fermi function $n_{i}(E)$, for electrode $i$ at low temperatures near Fermi energy $E_{F}$ marked by the dashed line.

$L(R)$. The tunnel Hamiltonian

$$
\hat{H}_{T}=\sum_{l, r} \mathcal{T}_{l, r} \hat{a}_{l}^{\dagger} \hat{c}_{r}+\text { H. c. }
$$

accounts for the electron jump between the reservoirs via the tunnel junction. Here, the first term describes the annihilation of an electron in $R$ with wave vector $r$ and the creation of an electron in $L$ with the wave vector $l$, leading to the transfer of an electron from $R$ to $L$. The term $\mathcal{T}_{l, r}$ is the corresponding tunnel matrix element. The second term is the hermitian conjugate (H. c.) of the first term and accounts for the transfer of electrons from $L$ to $R$.

When an electron is transferred from one electrode to another, it carries an electronic charge $(-e)$ and energy with itself. The change in the total charge of the electrodes leads to an electrical current while the change in the energy of the system leads to a heat current. The energy carried by the electron to the electrode after the transfer (for example, $\epsilon$ in Eqs. (2.8)) is quickly redistributed between the surrounding particles such as electrons and phonons and hence, it can be viewed as Joule heat associated with the transition.

Treating the tunnel Hamiltonian (2.2) as a perturbation, the golden rule calculation yields the transition rates as [64]

$$
\Gamma_{L \rightarrow R}(\Delta E)=\frac{1}{e^{2} \mathcal{R}} \int d \epsilon g_{L}(\epsilon) h_{R}(\epsilon-\Delta E) .
$$

Here, $\Delta E=-e V$ for a single tunnel junction with bias voltage $V$ but for a device with multiple junctions, such as a single-electron transistor and a double dot, $\Delta E$ is the change in electrostatic energy of the device after the 
electron tunneling. In the latter case, the individual junction resistance $\mathcal{R}$ should be much higher than the quantum resistance $R_{K}=h / e^{2} \approx 26 \mathrm{k} \Omega$ for the golden rule to be valid. In Eq. (2.3), the term $g_{i}(E)=n_{i}(E) f_{i}(E)$ is the product of the normalized density of states in electrode $i$, denoted by $n_{i}(E)=\rho_{i}(E) / \rho_{i}(0)$, and the Fermi function $f_{i}(E)=1 /\left[e^{E /\left(k_{B} T_{i}\right)}+1\right]$ which gives the distribution of electrons in the electrode $i$ at temperature $T_{i} . \rho_{i}(0)$ is the density of states (DOS) in electrode $i$ at Fermi energy $E_{F}$. Here and further in the thesis, $E$ is the energy counted with respect to the Fermi energy $E_{F} . \rho_{i}(E)$ is the DOS of electrode $i$ at energy $E$ with respect to its Fermi energy, and $h_{i}(E)=n_{i}(E)\left[1-f_{i}(E)\right]$. The tunnel resistance $\mathcal{R}=\hbar /\left[2 \pi e^{2}|\mathcal{T}|^{2} \rho_{l}(0) \rho_{r}(0)\right]$ is a combination of the densities of states in the electrodes and the tunnel amplitude $\mathcal{T}$ under the assumption that $\mathcal{T}_{l, r}$ does not vary with energy and momentum of the wave vectors $l$ and $r$.

The normalized density of states in a normal metal is $n_{N}(E)=1$ near the Fermi level $E_{F}$. In a superconductor, the quasiparticle density of states depends strongly on energy near $E_{F}$ (Fig. 2.1 (b)). This dependence is given by the Bardeen, Cooper and Schrieffer (BCS) theory of superconductivity $[76,77]$

$$
n_{S}(E)=\frac{|E|}{\sqrt{E^{2}-\Delta^{2}}} \theta(|E|-\Delta),
$$

where the normalization is with respect to the density of states of the superconductor in its normal state at the Fermi level ${ }^{1}$. The superconductor energy gap $\Delta$ decreases with an increase in temperature and reaches 0 at the critical temperature $T_{\mathrm{c}}$ of the superconductor. The value of $\Delta$ can be determined in experiments from the $I-V$ characteristics of the junction, as $2 \Delta$ is the amount of energy needed to break a Cooper pair and create two quasiparticles. These quasiparticles are responsible for carrying heat in superconductors.

We define $I_{i \rightarrow j}^{k \rightarrow l}$ as the average current from $i \rightarrow j$ due to the transfer of electrons from $k \rightarrow l$. As each electron carries $-e$ charge with it, the average current from $L \rightarrow R$ caused by the transfer of electrons from $L \rightarrow R$ is the product of the electronic charge and the transition rate, i.e., $I_{L \rightarrow R}^{L \rightarrow R}=-e \Gamma_{L \rightarrow R}\left(\Delta E_{L \rightarrow R}\right)$. Here, $\Delta E_{L \rightarrow R}=\Delta E$, the subscript is added to emphasize the difference in energy cost for an electron jump from $L \rightarrow R$ and backwards. Similarly, the average charge current from $L \rightarrow R$ due to the electron jumps from $R \rightarrow L, I_{L \rightarrow R}^{R \rightarrow L}=e \Gamma_{R \rightarrow L}\left(\Delta E_{R \rightarrow L}\right)$. Since the energy cost is computed as the difference in total energy of the system before and after the transition of electron, we have $\Delta E_{R \rightarrow L}=-\Delta E_{L \rightarrow R}=-\Delta E$. Combining these, the net current through the junction from $L \rightarrow R$ is

$$
I_{L \rightarrow R}^{\text {net }}=I_{L \rightarrow R}^{L \rightarrow R}+I_{L \rightarrow R}^{R \rightarrow L}=-e\left[\Gamma_{L \rightarrow R}(\Delta E)-\Gamma_{R \rightarrow L}(-\Delta E)\right] .
$$

The rates can be computed using Eq. (2.3). For computing $\Gamma_{R \rightarrow L}$ from

\footnotetext{
${ }^{1}$ For $\mathrm{Al}$, the density of states in its normal state at Fermi level is $1.80 \pm 0.35 \times$ $10^{47} \mathrm{~m}^{-3} \mathrm{~J}^{-1}[78-82]$.
} 
Eq. (2.3), we switch the role of $L$ and $R$ electrodes, namely,

$$
\Gamma_{R \rightarrow L}(\Delta E)=\frac{1}{e^{2} \mathcal{R}} \int d \epsilon g_{R}(\epsilon) h_{L}(\epsilon-\Delta E) .
$$

When both leads are at the same temperature $T$, the transition rates follow the detailed balance

$$
\frac{\Gamma_{L \rightarrow R}(\Delta E)}{\Gamma_{R \rightarrow L}(-\Delta E)}=e^{-\Delta E /\left(k_{B} T\right)} .
$$

Based on the golden rule calculation, the average heat currents generated in the reservoirs $L$ and $R$ by the transition $L \rightarrow R$ are

$$
\begin{aligned}
& \dot{Q}_{L \rightarrow R}^{L}(\Delta E)=\frac{1}{e^{2} \mathcal{R}} \int d \epsilon(-\epsilon) g_{L}(\epsilon) h_{R}(\epsilon-\Delta E) \text { and } \\
& \dot{Q}_{L \rightarrow R}^{R}(\Delta E)=\frac{1}{e^{2} \mathcal{R}} \int d \epsilon(\epsilon-\Delta E) g_{L}(\epsilon) h_{R}(\epsilon-\Delta E) .
\end{aligned}
$$

Similarly, the average heat currents generated by the transition $R \rightarrow L$ are

$$
\begin{aligned}
& \dot{Q}_{R \rightarrow L}^{L}(\Delta E)=\frac{1}{e^{2} \mathcal{R}} \int d \epsilon \epsilon g_{R}(\epsilon) h_{L}(\epsilon-\Delta E) \text { and } \\
& \dot{Q}_{R \rightarrow L}^{R}(\Delta E)=\frac{1}{e^{2} \mathcal{R}} \int d \epsilon(\Delta E-\epsilon) g_{R}(\epsilon) h_{L}(\epsilon-\Delta E)
\end{aligned}
$$

in $L$ and $R$, respectively. The total heat released

$$
\dot{Q}^{\text {tot }}=\sum_{i=L, R}\left[\dot{Q}_{L \rightarrow R}^{i}(\Delta E)+\dot{Q}_{R \rightarrow L}^{i}(\Delta E)\right]=I_{L \rightarrow R}^{\text {net }} V .
$$

This heat current in the system is associated to a change in the entropy of the system, with the rate of entropy production $\dot{S}^{\text {tot }}=I_{L \rightarrow R}^{\text {net }} V / T$ for the tunnel junction. We discuss the entropy production due to the flow of electrons through the double dot and its connection to heat in Chapter 5.

In the following sections, we present briefly different devices that have been used in the experiments carried out for this thesis. All the devices discussed below are made of NIS tunnel junctions to suppress the rate of electron transfer through the junction at low biasing conditions because of the presence of a superconductor with BCS density of states on one side of the junction. This facilitates the counting of single-electron transitions using a single-electron transistor (SET) [13], a technique employed in all the measurements presented in this thesis.

\subsection{Single-electron box}

In this section, we give a brief introduction to the simplest single-electron device, a single-electron box (SEB), see for example Ref. [83]. It consists of 
a metal lead tunnel-coupled to an island. A scanning electron micrograph (SEM) of a SEB is shown in Fig. 2.2 (a) where the lead and island are colored in green and turquoise, respectively. In the figure, the island of the SEB is capacitively coupled to a voltage source $V_{g}$ via a capacitance $C_{g}$, and the lead is connected to the common ground. This is a typical setup used to investigate such a system. In the experiments, an SET is capacitively coupled to the island whose charge population we are interested in. We discuss such a setup in the next section.
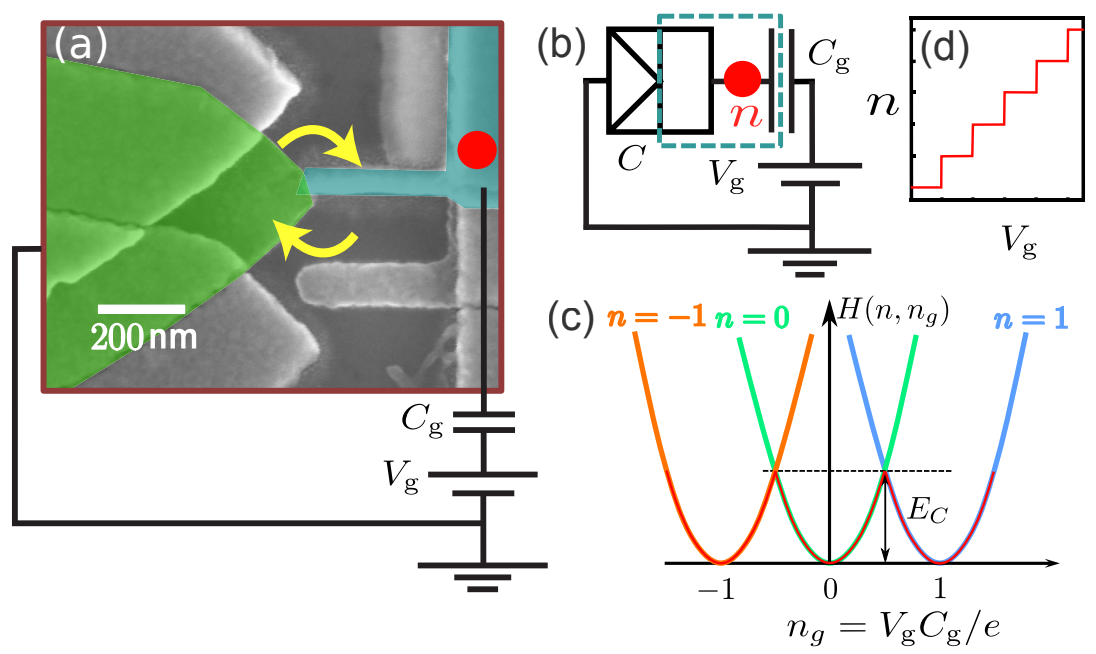

Figure 2.2. (a) Pseudo-colored SEM image of a single-electron box with its electrical connections. The turquoise normal metal island is capacitively coupled (capacitance $C_{\mathrm{g}}$ ) to a voltage source $V_{g}$ and the green superconducting lead is connected to a common ground. The electron (red circle, not to scale) on the island can jump to the lead and back (sketched by yellow arrows) through a tunnel junction of capacitance $C$ when $V_{g}$ is applied. (b) Schematic of the electronic circuit in (a). The turquoise dashed box is the island with $n$ as the number of extra electrons on the island. (c) The variation of SEB energy with change in the gate voltage for different $n$ values mentioned in the legend. The red curve shows the variation of $n$ with $V_{\mathrm{g}}$, corresponding to the minimum energy of the SEB. (d) The change in the number of extra electrons on the island $n$ with an increase in the gate voltage.

The total energy of an SEB (for example, in Fig. 2.2) is given by

$$
E\left(n, n_{g}\right)=E_{C}\left(n-n_{g}\right)^{2} .
$$

Here, $n$ is the number of extra electrons, compared to the charge neutrality, on the island at a given value of the gate voltage $V_{\mathrm{g}}$, and we define the "gate charge" $n_{g}=C_{\mathrm{g}} V_{\mathrm{g}} / e$. The scale of the charging energy is $E_{C}=$ $e^{2} / 2\left(C+C_{\mathrm{g}}\right)$, where, $C$ is the capacitance of the tunnel junction in SEB. In the experiments, the capacitance of a typical SEB is $C+C_{\mathrm{g}} \sim 1 \mathrm{fF}$. This ensures that the charging energy $E_{C} / k_{B} \sim 1 \mathrm{~K}$ is much larger than the thermal energy $k_{B} T$, a condition that needs to be satisfied for the singleelectron effects in the device to be dominant. This condition is satisfied for all the devices used in this thesis when subjected to sub-K temperatures. 
The energy cost to transfer an electron onto (out of) the island $\Delta E_{n \rightarrow n+1}$ $\left(\Delta E_{n \rightarrow n-1}\right)$ can be calculated using Eq. (2.10) as

$$
\Delta E_{n \rightarrow n \pm 1}=E\left(n \pm 1, n_{g}\right)-E\left(n, n_{g}\right)=E_{C}\left[1 \pm 2\left(n-n_{g}\right)\right] .
$$

The transition rates for electron transfer to and from the island can be computed using these energy costs in expressions (2.3) and (2.6), respectively. These transition rates follow the detailed balance, as expected from (2.7), when both the lead and the island are at the same temperature.

In Fig. 2.2 (c), we plot the dependence of the total energy (2.10) on $n_{g}$ for fixed values of $n$ (presented in different colors). When $k_{B} T \ll E_{C}$ and $n_{g}$ is close to $1 / 2$, the island is populated with 0 or 1 extra electrons. Upon a change in the gate charge by 1 , for example between $n_{g}=0$ and 1 , the $n$ corresponding to minimum energy changes from no extra electron to one extra electron on the island. The system is switching between the two charge states, $n=0$ and 1 , and the probability of the system to occupy the third state is given by $e^{-E_{C}\left(2-\max \left(n_{g}, 1-n_{g}\right)\right)^{2} / k_{B} T} / Z$, where the partition function $Z=\sum_{m} e^{-E\left(m, n_{g}\right) /\left(k_{B} T\right)}$ takes care of the normalization. This probability is exponentially suppressed at $k_{B} T \ll E_{C}$, making the SEB a two-state system. This is the temperature regime used for the devices presented in this thesis.

In the following section, we briefly discuss a single-electron transistor. We demonstrate how to use it as a single-electron box and as a detector to count the electron transfer in another single-electronic device capacitively coupled to it.

\subsection{Single-electron transistor}

A single-electron transistor (SET) comprises of two tunnel junctions connected in series. For example, the blue colored structure in Fig. 2.3 (a) shows the scanning electron micrograph of an SET. The structure enclosed in blue box in panel (b) illustrates the electronic circuit corresponding to the SET. Figure 2.3 shows the two capacitively coupled SETs, one used as a device and another one as a detector (blue structures). The change in the number of extra electrons $n$ on the turquoise island of the device is inferred using the output current through the detector SET [74, 84-93]. In the further text, this sample is also called as a single-dot or single-island structure, due to the presence of only one device island in it.

Figure 2.3 (b) shows the schematic sketch of the device and detector SETs, along with the measurement setup used in Publications II and I. The $V_{\mathrm{b}}$ $\left(V_{\mathrm{b}, \mathrm{det}}\right)$ connected to the left lead of the SET is the DC bias voltage source for the device (detector). To measure the net current through the detector, its right lead is connected to a digital multimeter via an amplifier with a typical amplification factor of $10^{11} \mathrm{~V} / \mathrm{A}$. This net current through the SET 

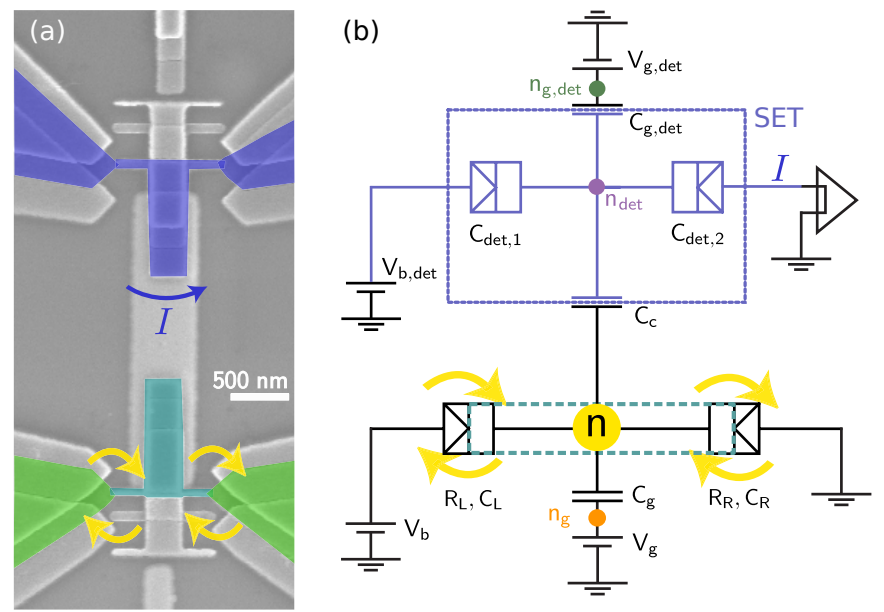

Figure 2.3. (a) Pseudo-colored scanning electron micrograph of two capacitively coupled single-electron transistors and (b) the corresponding electronic circuit of the experimental setup. The yellow arrows show the direction of electron transfer onto and out of the turquoise island. The SET with turquoise island is the device with green leads and the blue SET is used as a detector. The number of extra electrons on the device island $n$ is affected by the gate voltage $V_{\mathrm{g}}$, the bias voltage $V_{\mathrm{b}}$ and the charge state on the detector island $n_{\text {det }}$.

is used to determine its parameters such as the resistance, capacitance and charging energy, see Sec. 3.2 for details. The theory of SETs has been developed in Refs. [70, 71, 94, 95].

To understand the dependence of the current on these parameters, we first introduce the charging energy of the detector SET (uncoupled from any other system) as

$$
E_{\mathrm{ch}, \mathrm{det}}\left(n_{\mathrm{det}}\right)=E_{C_{\mathrm{det}}}\left(n_{\mathrm{det}}-n_{\mathrm{g}, \mathrm{det}}\right)^{2},
$$

where the scale of the charging energy $E_{C_{\text {det }}}=e^{2} /\left(2 C_{\Sigma \text {,det }}\right)$ depends on the total capacitance $C_{\Sigma, \mathrm{det}}=C_{\mathrm{det}, 1}+C_{\mathrm{det}, 2}+C_{\mathrm{g}, \mathrm{det}}+C_{\mathrm{c}}$. The number of extra electrons on the island $n_{\text {det }}$ is influenced by the gate induced charge $n_{\mathrm{g}, \mathrm{det}}=$ $C_{\mathrm{g}, \mathrm{det}} V_{\mathrm{g}, \mathrm{det}} / e$. The net current through an SET is computed in a similar manner as for a tunnel junction (2.5) with the energy cost of electron transfer given by the change in its total energy $E_{\text {det }}(2.13)$ corresponding to the electron jump. The total energy is a sum of the charging energy $E_{\text {ch,det }}\left(n_{\text {det }}\right)$ and the energy contribution due to the potential drop across its two leads,

$$
E_{\operatorname{det}}\left(n_{\text {det }}\right)=E_{\text {ch,det }}\left(n_{\operatorname{det}}\right)+N_{\text {det }, \mathrm{L}} e V_{\text {det }, \mathrm{L}}+N_{\text {det }, \mathrm{R}} e V_{\operatorname{det}, \mathrm{R}} .
$$

Here, $N_{\text {det,L }}\left(N_{\text {det,R }}\right)$ is the total number of electrons in the left (right) lead of the SET and $V_{\text {det,L }}\left(V_{\text {det,R }}\right)$ is the potential of its left (right) lead.

The total charging energy of a system with a device SET capacitively coupled to a detector SET (Fig. 2.3) is given by the sum of the charging 
energies of the individual SETs and their coupling energy, namely

$$
E(n)=E_{C}\left(n-n_{g}\right)^{2}+E_{C_{\operatorname{det}}}\left(n_{\operatorname{det}}-n_{\mathrm{g}, \mathrm{det}}\right)^{2}+2 E_{c, \operatorname{det}}\left(n-n_{g}\right)\left(n_{\mathrm{det}}-n_{\mathrm{g}, \mathrm{det}}\right) .
$$

The first term is the charging energy of the device, where $E_{C}=e^{2} /\left(2 C_{\Sigma}\right)$, the total device capacitance is $C_{\Sigma}=C_{\mathrm{L}}+C_{\mathrm{R}}+C_{\mathrm{g}}+C_{\mathrm{c}}, n$ is the number of extra electrons on the island of the device at the gate voltage $V_{\mathrm{g}}$, and $n_{\mathrm{g}}=C_{\mathrm{g}} V_{\mathrm{g}} / e$. The second term is the charging energy of the detector (2.12) and the last term is the coupling energy between the device and detector, where $E_{c, \text { det }}=e^{2} C_{c} /\left[2\left(C_{\Sigma} C_{\Sigma, \text { det }}-C_{c}^{2}\right)\right]$ depends on the coupling capacitance $C_{c}$, the device capacitance $C_{\Sigma}$ and the detector capacitance $C_{\Sigma \text {,det }}$. The last term governs how strong is the influence of the device on the detector and vice versa. This property of capacitive coupling enables us to use an SET as a charge detector.

The total energy of the system is given by

$$
E_{\text {tot }}\left(n, N_{L}, N_{R}\right)=E(n)+N_{L} e V_{L}+N_{R} e V_{R}+N_{\text {det }, \mathrm{L}} e V_{\text {det }, \mathrm{L}}+N_{\text {det, } \mathrm{R}} e V_{\text {det, } \mathrm{R}},
$$

where the first term is the total charging energy of the system (2.14) and the second and third terms are the contributions due to the potential drop across the device. The total number of electrons in the left (right) lead of the device SET is $N_{L}\left(N_{R}\right)$ and the potential of its left (right) lead is $V_{L}\left(V_{R}\right)$. In the experiments, there is no potential drop across the device, i.e., $V_{L}=V_{R}=0$, resulting in a total energy (2.15) equivalent to $E(n)(2.14)$ plus the energy contributions due to the potential drop across the detector.

The energy costs of electron transfer onto $(n \rightarrow n+1)$ and out of $(n \rightarrow n-1)$ the device island are given by the change in its total energy (2.15), namely

$$
\begin{aligned}
& \Delta E_{n \rightarrow n \pm 1}^{L}=E_{\text {tot }}\left(n \pm 1, N_{L} \mp 1, N_{R}\right)-E_{\text {tot }}\left(n, N_{L}, N_{R}\right) \\
& \Delta E_{n \rightarrow n \pm 1}^{R}=E_{\text {tot }}\left(n \pm 1, N_{L}, N_{R} \mp 1\right)-E_{\text {tot }}\left(n, N_{L}, N_{R}\right) .
\end{aligned}
$$

Here, the superscript $L(R)$ implies the energy cost of electron transfer through the left (right) junction of the device. Using these energy costs (2.16) and Eqs. (2.3), (2.5) and (2.6), the rate of electron transfer $\Gamma_{n \rightarrow n \pm 1}^{\alpha}$ and the net current through the device $I=I_{\alpha}$ are computed. Here, $\alpha=L(R)$ for transitions through the left (right) junction. The rates are given by

$$
\begin{aligned}
& \Gamma_{n \rightarrow n \pm 1}^{L}=\frac{1}{e^{2} \mathcal{R}} \int d \epsilon g_{L}(\epsilon) h_{i}\left(\epsilon-\Delta E_{n \rightarrow n \pm 1}^{L}\right), \\
& \Gamma_{n \rightarrow n \pm 1}^{R}=\frac{1}{e^{2} \mathcal{R}} \int d \epsilon g_{i}(\epsilon) h_{R}\left(\epsilon-\Delta E_{n \rightarrow n \pm 1}^{R}\right),
\end{aligned}
$$

where the subscripts $L, i$ and $R$ correspond to the left lead, island and right lead, respectively. The current from left to right through left and right junctions are $I_{L}=-e \sum_{n} p(n)\left[\Gamma_{n \rightarrow n+1}^{\alpha}-\Gamma_{n \rightarrow n-1}^{\alpha}\right]$ and $I_{R}=-e \sum_{n} p(n)\left[\Gamma_{n \rightarrow n-1}^{\alpha}-\right.$ 
$\left.\Gamma_{n \rightarrow n+1}^{\alpha}\right]$. The currents through the junctions in series are equal due to the conservation of charge. This gives the net current as

$$
I=-e \sum_{n} p(n)\left[\Gamma_{n \rightarrow n+1}^{L}-\Gamma_{n \rightarrow n-1}^{L}\right]=-e \sum_{n} p(n)\left[\Gamma_{n \rightarrow n-1}^{R}-\Gamma_{n \rightarrow n+1}^{R}\right],
$$

where $p(n)$ is the probability to find $n$ extra electrons on the device island at steady state, calculated using the master equation [96]

$\frac{d p(n, t)}{d t}=\Gamma_{n+1 \rightarrow n} p(n+1, t)+\Gamma_{n-1 \rightarrow n} p(n-1, t)-\left(\Gamma_{n \rightarrow n+1}+\Gamma_{n \rightarrow n-1}\right) p(n, t)$.

The steady state condition $d p(n, t) / d t=0$ imposes the condition on the probability $p(n, t)$ to be time-independent. Thus, we drop the time argument in the stationary probability $p(n)$ throughout the thesis.

The master equation (2.19) depicts the Markov dynamics of a system with two possible jumps in the charge state from a given state $n$, namely from $n \rightarrow n \pm 1$. For a system with arbitrarily many jumps allowed from a given state, the system dynamics is governed by a more generalized master equation [96]

$$
\frac{d p(n, t)}{d t}=\sum_{m(\neq n)} \Gamma_{m \rightarrow n}(t) p(m, t)-\left(\sum_{m(\neq n)} \Gamma_{n \rightarrow m}(t)\right) p(n, t) .
$$

Here, we use the shorthand notation $\Gamma_{m \rightarrow n}(t)$ for the transition rate from the initial state $m$ to the final state $n$ and $p(n, t)$ is the occupation probability of the state $n$ at time $t$. The time dependence in the transition rates could be due to a controlled drive in the system or an uncontrolled fluctuation in rates due to detector back-action. For the system at steady state, such as the experiments on an SET and a double dot presented in this thesis, the rates are considered to be time-independent.

The master equation (2.20) recovers Eq. (2.19) when we assume the rates to be independent of time and allow only transitions to two neighbouring states $n \pm 1$ from any state $n$. For a system with 4-probable states, the evolution equation (2.20) depicts the dynamics of its evolution with three probable transitions from each state. We discuss more about such a system in Sec. 2.4.

\subsubsection{Measurement protocol}

In the experiments, the bias voltage $V_{\mathrm{b}, \mathrm{det}}$ of the detector SET is fixed such that there is a maximum change in the detector output current $I$ when a jump in the system state $n$ occurs. Next, the gate voltage $V_{\mathrm{g} \text {,det }}$ of the detector is fixed such that a small change in $V_{\mathrm{g}, \mathrm{det}}$ induces the maximum change in $I$, i.e., $\left|\partial I / \partial V_{\mathrm{g}, \text { det }}\right|$ is maximized.

Both the device leads are connected to a common ground $V_{\mathrm{b}}=0$. This ensures both the leads of the device to be at the same potential. This 
implies that the two leads of the device SET can be approximated as a huge lead connecting to its island from two sides, thus making the device SET act like an SEB.

During the measurements, the gate induced charge $n_{\mathrm{g}}$ is kept fixed to acquire a long time trace of the current $I$, around 48 hours with the transition rate of electron jumps in device SET of the order of $100 \mathrm{~Hz}$. This ensures a large number of electrons jumping onto and out of the device island, hence improving the statistics of the fluctuations, see for example Fig. 4.4 and Publication II. The gate voltage $V_{\mathrm{g}}$ is used to control the rate of electron transfer onto and out of the device island. In Figure 4.5, we present the results with varying rates due to change in $V_{g}$.

The measurement output is the time trace from the detector SET. Figure 4.1 (a) shows one such trace. The high (low) level in the time trace is interpreted as the presence (absence) of one extra electron on the turquoise island. However, this does not give us information on the transition of electrons into or out of the individual leads of the device. That is, given the jump of an electron from a lead to the island, we cannot distinguish whether the electron jump has occurred from the left lead or the right one. This limitation is surpassed in an electronic double dot where we can check the direction of electron transfer once an electron jump has occurred. This is the focus of the next section.

\subsection{Electronic double dot system}

An electronic double dot [85, 97, 98] comprises of two metallic islands tunnel-coupled to two leads and to each other. The orange box in Fig. 2.4 (a) shows the electron micrograph of a double dot structure with green $(\mathrm{N})$ and cyan (S) islands tunnel-coupled to each other and to the red (S) and purple (N) leads. Panel (b) shows the corresponding electronic circuit together with the room-temperature measurement setup. As the double dot consists of two islands, it can be approximated as a system with four states. As mentioned earlier, all the tunnel junctions are of the NIS type. The top green and bottom cyan SETs in the figure are used as detectors, each capacitively coupled to the island of same color.

The output currents $I_{\mathrm{det}, \mathrm{L}}$ and $I_{\mathrm{det}, \mathrm{R}}$ through these detectors are used to infer the charge state of the system, i.e., the number of extra electrons on the islands of the double dot. For example, in Fig. 2.5 (a), we present the color map of the rescaled ${ }^{2}$ detector output currents, $i_{L}$ and $i_{R}$, at fixed double dot bias voltage $V_{\mathrm{b}}$ and gate voltages $V_{\mathrm{g}, \mathrm{L}}$ and $V_{\mathrm{g}, \mathrm{R}}$. The figure shows four densely populated system states $\left(n_{L}, n_{R}\right)=\{(0,0),(0,1),(1,0),(1,1)\}$ with their probability density marked by the scale on right. Here, $n_{L}\left(n_{R}\right)$

\footnotetext{
$2_{\text {The rescaled current is defined as }} i_{\alpha}=\left[I_{\operatorname{det}, \alpha}-I_{\operatorname{det}, \alpha}^{\min }\right] /\left[I_{\operatorname{det}, \alpha}^{\max }-I_{\operatorname{det}, \alpha}^{\min }\right]$, where $I^{\max }$ $\left(I^{\mathrm{min}}\right)$ is the average value of the current $I$ corresponding to state $n_{\alpha}=1\left(n_{\alpha}=0\right)$.
} 


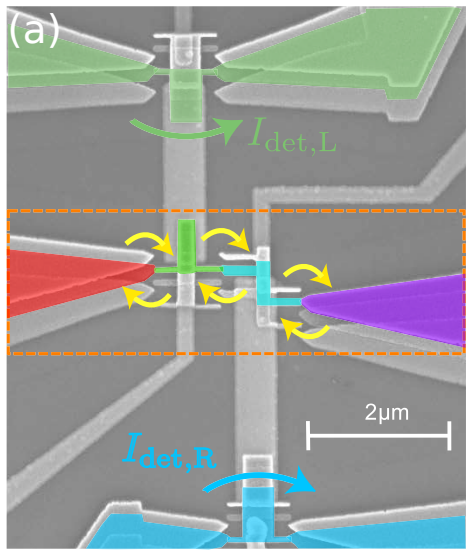

(b)

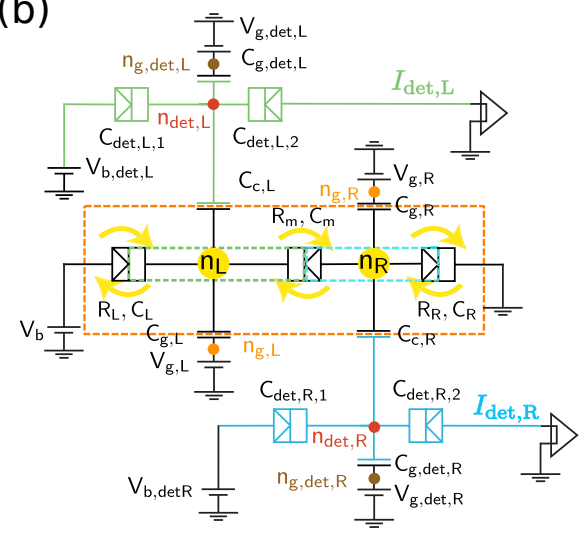

Figure 2.4. (a) Sample micrograph of a metallic double dot with false color to identify its different components. (b) Schematic of the electronic circuit corresponding to the sample together with the measurement setup. The double dot part of the sample (enclosed in the orange box) consists of two islands (green and cyan structures) and two leads (red and purple). The leads and islands are tunnel coupled to each other forming three NIS tunnel junctions in series, with capacitances $C_{L}, C_{m}, C_{R}$ and resistances $R_{L}, R_{m}, R_{R}$, corresponding to the left, middle and right junction respectively. The electrons on each island can transfer to the neighboring island or lead, shown by the yellow arrows. These jumps within the islands and between the island and lead are monitored using two single-electron transistors (SETs), one capacitively coupled to each island. The coupling capacitances are $C_{\mathrm{c}, \mathrm{L}}$ and $C_{\mathrm{c}, \mathrm{R}}$ respectively for the left and right island-detector couplers, gray rectangular structures between the islands and SETs. The remaining gray structures are the gates to the double dot and the detectors, each connected to a DC voltage source, $V_{\mathrm{g}, \mathrm{L}}, V_{\mathrm{g}, \mathrm{R}}, V_{\mathrm{g}, \text { det.L }}$ and $V_{\mathrm{g}, \mathrm{det}, \mathrm{R}}$ via the capacitances, $C_{\mathrm{g}, \mathrm{L}}, C_{\mathrm{g}, \mathrm{R}}, C_{\mathrm{g}, \mathrm{det}, \mathrm{L}}$ and $C_{\mathrm{g}, \mathrm{det}, \mathrm{R}}$, respectively. The capacitances of the detector junctions are $C_{\mathrm{det}, \mathrm{L}, 1}, C_{\mathrm{det}, \mathrm{L}, 2}, C_{\mathrm{det}, \mathrm{R}, 1}$, and $C_{\text {det,R,2. }}$. The DC voltages $V_{\mathrm{b}}, V_{\mathrm{b}, \mathrm{det}, \mathrm{L}}$, and $V_{\mathrm{b}, \mathrm{det}, \mathrm{R}}$ are applied across the double dot and detectors, respectively. Adapted from Publication III.

(a)

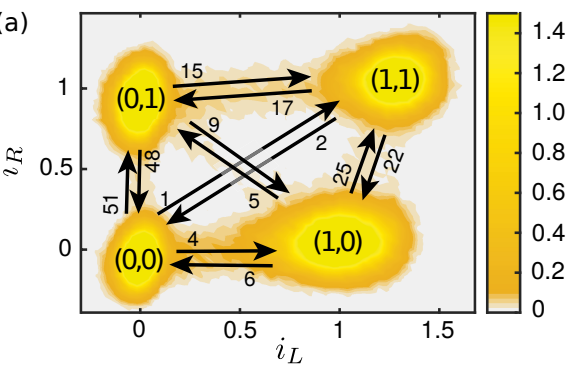

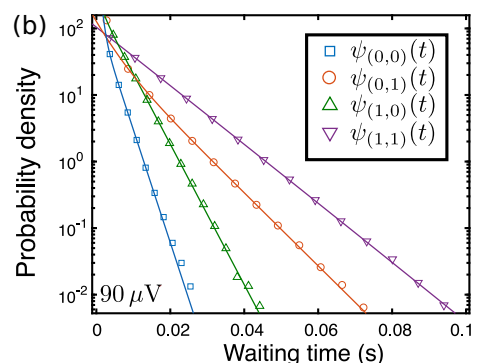

Figure 2.5. (a) The probability density of the normalized detector currents $\left(i_{L}, i_{R}\right)$ obtained from a $15 \mathrm{~s}$ time trace for double dot bias $V_{\mathrm{b}}=90 \mu \mathrm{V}$, showing the four densely populated charge states $\left(n_{L}, n_{R}\right)$. The possible transitions between the states are represented by the black arrows with the numbers indicating the number of jumps per second, averaged over 7.5 hours. (b) The measured (symbols) waiting-time distributions $\psi_{\left(n_{L}, n_{R}\right)}(t)$ for the states $\left(n_{L}, n_{R}\right)$ in the double dot at bias $V_{\mathrm{b}}=90 \mu \mathrm{V}$. The solid lines are exponential fits to the experimental values (symbols). Adapted from Publication III. 
is the number of extra electrons on the left (right) island of the double dot. The black arrows indicate the transitions between different system states, with the numbers presenting the average number of jumps in a second.

In Fig. 2.5 (b), we plot the waiting-time distributions $\psi_{\left(n_{L}, n_{R}\right)}(t)$ for the four system states shown in panel (a). The waiting-time distribution $\psi_{x}(t)$ is defined as the probability of system to stay in a given state $x$ for time duration $t$ before undergoing any transition. The exponential probability density confirms the Markovian dynamics of the system states. Hence, the time evolution of the system states is given by the master equation (2.20), where the transition rates between the different system states are governed by the system parameters, such as capacitances $C_{x}$ and resistances $R_{x}$, and the DC voltages applied to the leads $V_{x}$, see Fig. 2.4 (b). This dependence is explained below.

The following subsections are arranged as follows. First, we mention the dependence of total energy of the double dot system on the system parameters, see Sec. 2.4.1. Then, we present a brief note on the transition rates between different system states with a table consisting of the experimental rates, see Sec. 2.4.2. Next, a brief note on the detector output currents is mentioned (Sec. 2.4.3) followed by a discussion on the different physical quantities that can be computed using the output currents, see Sec. 2.4.4.

\subsubsection{Energy considerations}

The total energy of a double dot system with each of its island capacitively coupled to an SET (for example, the system in Fig. 2.4) is given by [97]

$$
\begin{aligned}
E\left(n_{L}, n_{R}\right)= & E_{\operatorname{dot}}\left(n_{L}, n_{R}\right)+E_{C, \operatorname{det}, L}\left(n_{\operatorname{det}, L}-n_{g, \operatorname{det}, L}\right)^{2} \\
& +E_{C, \operatorname{det}, R}\left(n_{\operatorname{det}, R}-n_{g, \operatorname{det}, R}\right)^{2} \\
& +E_{c, L}\left(n_{L}-n_{g, L}\right)\left(n_{\operatorname{det}, L}-n_{g, \operatorname{det}, L}\right) \\
& +E_{c, R}\left(n_{R}-n_{g, R}\right)\left(n_{\operatorname{det}, R}-n_{g, \operatorname{det}, R}\right)+\frac{N_{L}-N_{R}}{2} e V_{\mathrm{b}} .
\end{aligned}
$$

The number of extra electrons on the island of the left (right) detector SET is $n_{\text {det,L }}\left(n_{\text {det, } \mathrm{R}}\right)$ and the total number of electrons in the left (right) lead is $N_{L}\left(N_{R}\right)$. The dimensionless gate induced charges of the metallic islands read

$$
\begin{aligned}
n_{g, \mathrm{det}, L} & =\frac{C_{g, \mathrm{det}, L} V_{g, \mathrm{det}, L}}{e}, n_{g, \operatorname{det}, R}=\frac{C_{g, \mathrm{det}, R} V_{g, \mathrm{det}, R}}{e}, \\
n_{g, L} & =\frac{C_{g, L} V_{\mathrm{g}, \mathrm{L}}}{e}+\frac{C_{L} V_{\mathrm{b}}}{2 e}, n_{g, R}=\frac{C_{g, R} V_{\mathrm{g}, \mathrm{R}}}{e}-\frac{C_{R} V_{\mathrm{b}}}{2 e} .
\end{aligned}
$$

Here, we have considered a symmetric bias of the double dot, i.e., the potentials of its left and right lead are $V_{\mathrm{b}} / 2$ and $-V_{\mathrm{b}} / 2$, respectively. In the experiments, we apply an asymmetric bias, i.e., the potential of the left lead is $V_{\mathrm{b}}$ while the right lead is grounded. The effect of this asymmetry on the dimensionless charge (2.22) and the total energy of the system (2.21) can be absorbed into shifts of the gate voltages $V_{\mathrm{g}, \mathrm{L}}$ and $V_{\mathrm{g}, \mathrm{R}}$. 
The first term in Eq. (2.21) is the electrostatic energy of the double dot, given by

$E_{\mathrm{dot}}\left(n_{L}, n_{R}\right)=\frac{E_{C 1}}{2}\left(n_{L}-n_{g, L}\right)^{2}+\frac{E_{C 2}}{2}\left(n_{R}-n_{g, R}\right)^{2}+E_{C, m}\left(n_{L}-n_{g, L}\right)\left(n_{R}-n_{g, R}\right)$.

Here, the first two terms are the charging energies of the islands with the scaling factors $E_{C 1}=e^{2} C_{2} / C_{0}^{2}$ and $E_{C 2}=e^{2} C_{2} / C_{0}^{2}$ determined by the effective capacitance of both islands ${ }^{3}, C_{1}$ and $C_{2}$, and the capacitance combination $C_{0}^{2}=C_{1} C_{2}-C_{m}^{2}$. The coupling energy is $E_{C, m}=e^{2} C_{m} / C_{0}^{2}$.

The second and third terms in Eq. (2.21) are the electrostatic energies of the detectors, where $E_{C, \operatorname{det}, \alpha}=e^{2} /\left[2 C_{\Sigma, \alpha}\right]$ is the charging energy of detector, $\alpha=L$ and $R$, with total capacitance $C_{\Sigma, \alpha}=C_{\mathrm{det}, \alpha, 1}+C_{\mathrm{det}, \alpha, 2}+C_{g, \operatorname{det}, \alpha}+$ $\left[C_{c, L}^{-1}+\left(C_{L}+C_{g, L}+C_{m}\right)^{-1}\right]^{-1}$.

The fourth and fifth terms in Eq. (2.21) describe the capacitive coupling between the dots and the detectors, where the coupling energies $E_{c, L}, E_{c, R}$ are defined as

$$
\begin{aligned}
& E_{c, R}=\frac{2 e^{2} C_{c, R}}{C_{2} C_{\Sigma, R}+\sqrt{C_{2} C_{\Sigma, R}\left(C_{2} C_{\Sigma, R}+4 C_{c, R}^{2}\right)}}, \\
& E_{c, L}=\frac{2 e^{2} C_{c, L}}{C_{1} C_{\Sigma, L}+\sqrt{C_{1} C_{\Sigma, L}\left(C_{1} C_{\Sigma, L}+4 C_{c, L}^{2}\right)}} .
\end{aligned}
$$

The last term in Eq. (2.21) is the energy contribution due to the potential drop across the double dot.

We define $n \equiv\left(n_{L}, n_{R}\right)$ to present the state of the double dot system, i.e., the state with $n_{L}\left(n_{R}\right)$ extra electrons on the left (right) island. The energy cost for the system to jump from a state $n$ to $m \equiv\left(m_{L}, m_{R}\right)$ is given by

$$
\Delta E_{n \rightarrow m}=E\left(m_{L}, m_{R}\right)-E\left(n_{L}, n_{R}\right) .
$$

From Eq. (2.25), we have $\Delta E_{n \rightarrow m}=-\Delta E_{m \rightarrow n}$. Thus, we need only six energy costs to characterize all the 12 transitions in the double dot, see the black arrows in Fig. 2.5 (a). The complete list of all six energy costs can be computed using Eqs. (2.21) and (2.25). It can be found in the supplemental material of Publication III, where this energy cost is referred to as the Joule heat $Q_{m}^{n}$ associated with the transition $m \rightarrow n$.

Using the energy cost (2.25), we can compute the transition rates between states, $\Gamma_{n \rightarrow m}$, and the net current through the double dot in a similar manner as for the tunnel junction, see Eqs. (2.3), (2.5) and (2.6). However, the energy cost $\Delta E_{n \rightarrow m}$, transition rate $\Gamma_{n \rightarrow m}$ and the net current calculated this way depend on the charge states of the detectors. For example,

\footnotetext{
${ }^{3}$ The effective capacitances of the double dot islands are $C_{1}=C_{L}+C_{g, L}+C_{m}+$ $\left[C_{c, L}^{-1}+\left(C_{\operatorname{det}, L, 1}+C_{\operatorname{det}, L, 2}+C_{g, \operatorname{det}, L}\right)^{-1}\right]^{-1}$ and $C_{2}=C_{R}+C_{g, R}+C_{m}+\left[C_{c, R}^{-1}+\right.$ $\left.\left(C_{\mathrm{det}, R, 1}+C_{\mathrm{det}, R, 2}+C_{g, \mathrm{det}, R}\right)^{-1}\right]^{-1}$.
} 
for the system to jump from $(0,1)$ to $(1,0)$ it reads

$$
\begin{aligned}
\Delta E_{n \rightarrow m}= & -\left(E_{C 1}-E_{C, m}\right)\left(\frac{1}{2}-n_{g, L}\right)+\left(E_{C 2}-E_{C, m}\right)\left(\frac{1}{2}-n_{g, R}\right) \\
& -E_{c, L}\left(n_{\operatorname{det}, L}-n_{g, \operatorname{det}, L}\right)+E_{c, R}\left(n_{\operatorname{det}, R}-n_{g, \operatorname{det}, R}\right) .
\end{aligned}
$$

This energy cost depends on the instantaneous values of charge states of the detectors, $n_{\operatorname{det}, L}$ and $n_{\text {det }, R}$, and their gate induced charges, $n_{g, \operatorname{det}, L}$ and $n_{g, \text { det, } R}$, along with the system state and gate induced charges. These charge states of the detectors fluctuate in time with a typical frequency of $I_{\text {det }, \alpha}^{\text {meas }} / e \gtrsim 0.1 \mathrm{GHz}$ which is much higher than our data acquisition rate $f=25 \mathrm{kHz}$. Hence, we experimentally observe the current through the double dot and the transition rates between system states corresponding to the energy costs as an average over the detector charges. We present this averaged energy costs with the superscript avg, i.e.,

$$
\Delta E_{n \rightarrow m}^{\mathrm{avg}}=\left\langle\Delta E_{n \rightarrow m}\right\rangle_{n_{\mathrm{det}, L}, n_{\mathrm{det}, R}} .
$$

This is the energy cost we obtain by neglecting the fluctuations due to the detectors.

\subsubsection{Transition rates}

The averaged transition rates $\Gamma_{n \rightarrow m}^{\mathrm{avg}}$ is the transition rate $\Gamma_{n \rightarrow m}$ averaged over the detector charges, namely

$$
\Gamma_{n \rightarrow m}^{\mathrm{avg}}=\left\langle\Gamma_{n \rightarrow m}\left(\Delta E_{n \rightarrow m}\right)\right\rangle_{n_{\mathrm{det}, L}, n_{\mathrm{det}, R}} .
$$

From the experimental data, we compute the transition rate from state $n$ to $m$ using [85]

$$
\Gamma_{n \rightarrow m}^{\exp }=\frac{\mathcal{N}_{n \rightarrow m}}{p(n) \tau}
$$

Here, $\mathcal{N}_{n \rightarrow m}$ is the total number of jumps from state $n$ to $m$ during the measurement time $\tau$. The steady state probability of the states $p(n)$ is given by

$$
p(n)=\tau_{n} / \tau,
$$

where $\tau_{n}$ is the time for which system stays in state $n$ during time $\tau$. We present the list of transition rates $\Gamma_{n \rightarrow m}^{\exp }$ corresponding to different bias voltages $V_{\mathrm{b}}$ across the double dot in Table 2.1.

The non-averaged rates $\Gamma_{n \rightarrow m}$ satisfy the detailed balance condition

$$
\frac{\Gamma_{n \rightarrow m}}{\Gamma_{m \rightarrow n}}=e^{-\Delta E_{n \rightarrow m} /\left(k_{B} T\right)}
$$

at equilibrium with the same temperature $T$ for electrons, phonons, and noise. However, in the presence of back-action of the detectors the detailed balance with electronic temperature $T_{\mathrm{el}}$ does not hold for the averaged rates 
Physics of single-electron devices

\begin{tabular}{|c|c|c|c|c|c|c|c|c|c|c|c|c|}
\hline Bias & \multicolumn{10}{|c|}{ Transition rates $\Gamma_{n}^{m} \equiv \Gamma_{n \rightarrow m}^{\exp }$ (in Hz) } \\
\hline$(\mu V)$ & $\Gamma_{(0,0)}^{(0,1)}$ & $\Gamma_{(0,1)}^{(0,0)}$ & $\Gamma_{(0,0)}^{(1,1)}$ & $\Gamma_{(1,1)}^{(0,0)}$ & $\Gamma_{(0,0)}^{(1,0)}$ & $\Gamma_{(1,0)}^{(0,0)}$ & $\Gamma_{(0,1)}^{(1,1)}$ & $\Gamma_{(1,1)}^{(0,1)}$ & $\Gamma_{(1,0)}^{(1,1)}$ & $\Gamma_{(1,1)}^{(1,0)}$ & $\Gamma_{(0,1)}^{(1,0)}$ & $\Gamma_{(1,0)}^{(0,1)}$ \\
\hline 90 & 644 & 131 & 14 & 4 & 52 & 39 & 41 & 43 & 167 & 54 & 25 & 30 \\
\hline 65 & 120 & 71 & 3 & 2 & 51 & 30 & 34 & 35 & 88 & 98 & 46 & 21 \\
\hline 50 & 104 & 76 & 8 & 7 & 274 & 177 & 149 & 156 & 81 & 98 & 39 & 22 \\
\hline 25 & 73 & 98 & 1 & 3 & 37 & 27 & 24 & 38 & 55 & 144 & 41 & 20 \\
\hline-25 & 91 & 82 & 2 & 2 & 36 & 28 & 26 & 40 & 67 & 114 & 25 & 32 \\
\hline-50 & 101 & 72 & 10 & 9 & 371 & 287 & 205 & 252 & 78 & 87 & 21 & 36 \\
\hline
\end{tabular}

Table 2.1. The transition rates for the double dot jumping from state $n \rightarrow m, \Gamma_{n \rightarrow m}^{\exp }$, calculated from the experimental data for different device bias voltages. All the data are obtained from counting statistics of experimental traces for a duration of at least 1 hour.

$\Gamma_{n \rightarrow m}^{\text {avg }}(2.28)$ and the averaged energy costs $\Delta E_{n \rightarrow m}^{\text {avg }}$, i.e., $\Gamma_{n \rightarrow m}^{\text {avg }} / \Gamma_{m \rightarrow n}^{\text {avg }} \neq$ $\exp \left[-\Delta E_{n \rightarrow m}^{\mathrm{avg}} / k_{B} T_{\mathrm{el}}\right]$. This is the case in the double dot system, where we use the relation between the average entropy production rate $\langle\dot{S}\rangle$ and Joule heating $\langle I\rangle V_{\mathrm{b}}$,

$$
\langle I\rangle V_{\mathrm{b}}=T_{\text {eff }}\langle\dot{S}\rangle,
$$

to define an effective temperature $T_{\text {eff. }}$. The details on how to calculate the entropy production in this case is provided in Chapter 5 and the appendix of Publication III.

\subsubsection{Current through the double dot}

The net current through the double dot, averaged over the detector charges, can be computed using Eq. (2.5). This net current is compared with the experimentally measured current to characterize the double dot system. See Sec. 3.2 for details on the characterization method.

Figure 2.6 shows the gate dependence of the measured current through the double dot at bias voltage (a) $V_{\mathrm{b}}=0$ and (b) $V_{\mathrm{b}}=120 \mu \mathrm{V}$. The data presented here corresponds to the system configuration in which all the electrodes are made normal by applying magnetic field $H \approx 150 \mathrm{mT}$ perpendicular to the structure. The black lines in (a) show the honeycomb structure as expected from the energetics of the system [97]. The black line shows the gate positions where the system switches between two states and the triple points show the positions where the system switches between three possible states. With an increase in bias voltage, these triple points are enlarged to take the form of a triangle. For example, at $V_{\mathrm{b}}=120 \mu \mathrm{V}$ in panel (b), we observe two overlapping triangles marking the disappearance of the line between the two triple points.

In experiments, we fix the gate voltages of the double dot such that the system stays at point $P$ between two triple points. We present below the measurement protocol briefly. For more details, we refer to the appendix of Publication III. 

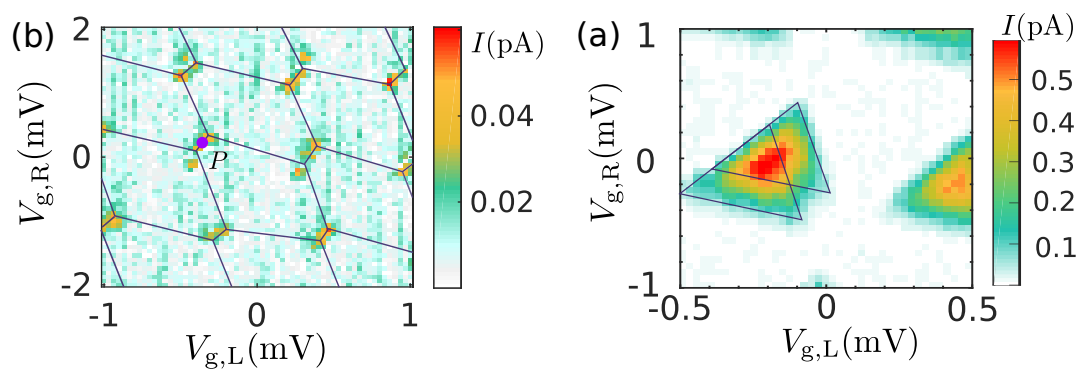

Figure 2.6. Current through the double dot structure at bias voltage (a) $V_{\mathrm{b}}=0$ and (b) $V_{\mathrm{b}}=120 \mu \mathrm{V}$ when all the electrodes are in the normal state. The black lines in (a) illustrate the honeycomb structure of the stable charge states, where the triple points are the gate voltage operating points with maximum current through the system. In (b) the black lines depict the broadening of the triple point when the bias voltage is increased. The marked location $P$ shows the midpoint of a line joining two triple points. The measurements were carried out at a similar point to have access to four approximately equally probable system charge states at non-zero bias.

\subsubsection{Measurement protocol}

A sketch of measurement setup is shown in Fig. 2.4 (b). In the experiments, at first the bias and gate voltages of the detectors are fixed to optimize the signal to noise ratio (SNR) in detector output currents, $I_{\mathrm{det}, \mathrm{L}}$ and $I_{\mathrm{det}, \mathrm{R}}$, at a fixed gate induced charges, $n_{L}$ and $n_{R}$, of the double dot. Next, the double dot bias voltage $V_{\mathrm{b}}$ is fixed while the right lead is left grounded. The two gates $V_{\mathrm{g}, \mathrm{L}}$ and $V_{\mathrm{g}, \mathrm{R}}$ are kept fixed such that the system is at the midpoint of the line connecting two triple points, for example point $P$ in Fig 2.6 (a). This ensures the accessibility of four states by the system at finite bias. The measurement is carried out for several hours with a feedback protocol to compensate for the low frequency background charge fluctuations [99, 100] which induce additional effective gate voltages to the double dot islands. The feedback checks the system state $\left(n_{L}, n_{R}\right)$ after every $15 \mathrm{~s}$ and provides a correction to the gates $V_{\mathrm{g}, \mathrm{L}}$ and $V_{\mathrm{g}, \mathrm{R}}$ once it realizes that the system is no longer at point $P$.

By the end of the measurement, we have recorded a few hours of time evolution of $I_{\text {det,L }}$ and $I_{\text {det,R }}$. Figure 2.7 (a) shows a sample of the detector output traces. We use such traces to infer the change in the charge state of the system $\left(n_{L}, n_{R}\right)$, see panel (b). The high (low) level of the detector current implies the presence (absence) of one extra electron on the corresponding island. Using the time dependence of these states $\left(n_{L}, n_{R}\right)$ and the definition (5.8), we compute the time trace of stochastic entropy production, see Fig. 2.7 (c). Since, the system states $\left(n_{L}, n_{R}\right)$ change between the four probable values (see Fig. 2.5), the computed stochastic entropy production has discrete steps without any experimental noise. Using the time trace of stochastic entropy production, we compute its minimum value the system can attain in a given time interval and study the statistics of such 


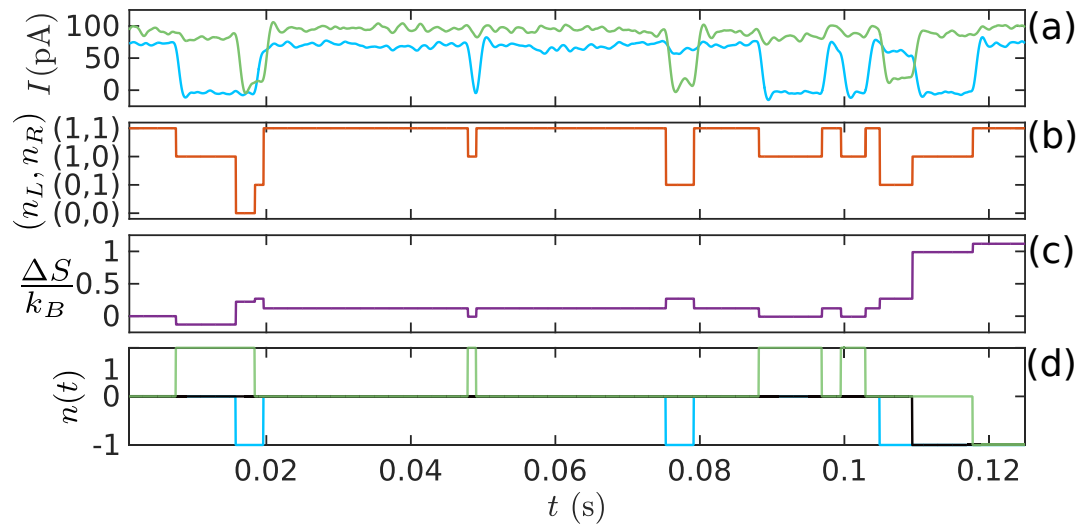

Figure 2.7. (a) A sample time trace of the current output from the left (right) detector, $I_{\text {det, } \mathrm{L}}\left(I_{\text {det, R }}\right)$ for device bias $V_{\mathrm{b}}=90 \mu \mathrm{V}$, shown by the green (cyan) line. (b) The corresponding time trace of system state $\left(n_{L}, n_{R}\right)$ calculated based on threshold detection and correlation analysis of the detector currents. (c) The time trace of stochastic entropy production corresponding to the time trace of $\left(n_{L}, n_{R}\right)$. (d) The time trace of net number of electrons transferred $n(t)$ through left (green), middle (black) and right (cyan) junction of the double dot. $n(t)$ is calculated as the total number of electrons transported from right to left minus the total number of charges transported in the reverse direction.

quantities. The computation of minimum stochastic entropy production from the corresponding time trace is demonstrated in Fig. 2.8 (a) and (b). For technical details, we refer to Chapter 5 and Publication III.

Furthermore, we use the time trace of system charge state (see Fig. 2.7 (b)) to compute the net charge transfered through each junction, see Fig. 2.7 (d). The time trace of this net charge transfered trace allows us to compute the first-passage times for certain amount of charge flow in a given direction. Fig. 2.8 (c) demonstrates the first-passage time (red circles) for sample experimental traces (blue) obtained from double-dot with bias voltage $90 \mu \mathrm{V}$. Such passage times are discussed in Chapter 6 and Publication IV. 

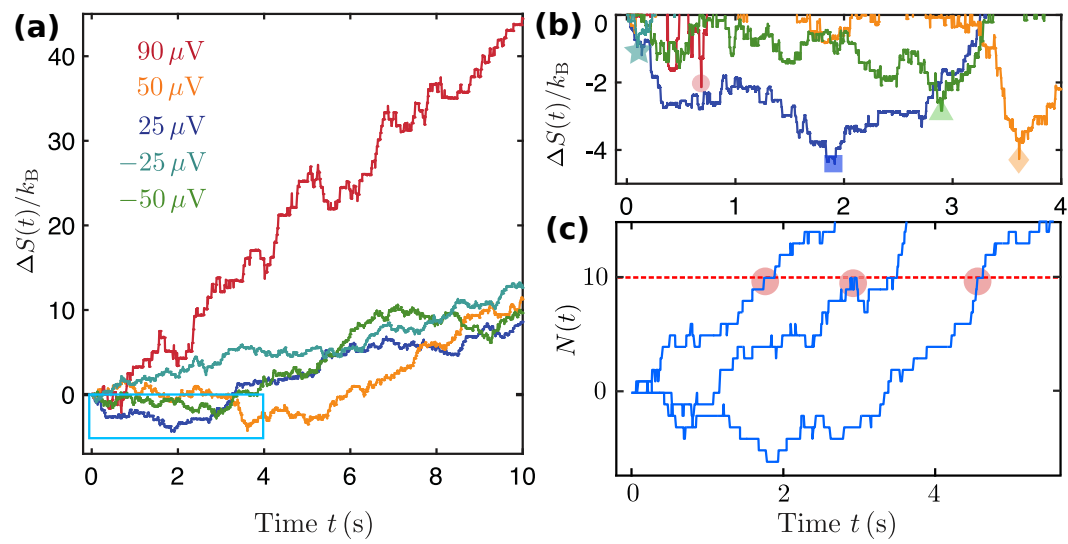

Figure 2.8. (a) Sample time trace of stochastic entropy production for different bias voltage across the double dot (see legend). (b) Zoomed view of blue rectangular region in (a). The different symbols show the minimum stochastic entropy produced for each trace in time $t=10 \mathrm{~s}$. (c) Sample time trace of the net number of electrons transferred $N(t)$ through middle junction of the double dot (in blue color) at bias voltage $90 \mu \mathrm{V}$. The red circles mark the first time $N(t)=10$, i.e., net 10 electrons pass through the middle junction of double dot system. Adapted from Publications III and IV. 


\section{Experimental methods}

In this chapter, we present the experimental methods employed to obtain the samples used in this work. The chapter starts with a brief introduction to device fabrication methods. Next, the procedures used to check the device functionality are discussed in Sec. 3.2. The chapter ends with a brief note on the measurement setup

\subsection{Sample fabrication}

Figures 3.1 (a) and (b) show scanning electron micrographs of samples with a single-island and a double-island structure, respectively. Different colors of the metal layers indicate different fabrication steps. The panels (i-xix) give a schematic overview of the steps used in the fabrication protocol, with parameters listed in Table 3.1. We will now go through each step in detail.

We start with a $525 \mu \mathrm{m}$ thick Si wafer coated with a thermally-grown $300 \mathrm{~nm}$ thick layer of $\mathrm{SiO}_{2}$, see Fig. 3.1 (i). The wafer is then spin-coated with an approximately $300 \mathrm{~nm}$ thick layer of positive e-beam resist (Allresist AR-P 6200, 13\% in anisole) using a spinner with 6000 rotations per minute (rpm) for $1 \mathrm{~min}$. After spinning, the polymer resist coated wafer is baked at $150^{\circ} \mathrm{C}$ for $10 \mathrm{~min}$ to evaporate the solvent and strengthen the resist layer, see Fig. 3.1 (ii).

Next, the resist is exposed to a $100 \mathrm{kV}$ electron beam (Vistec EBPG 5000+) to define the shapes of the features in the ground plane layer (orange structures in Fig. 3.1). The ground plane includes the coupler electrode between the device and detector parts of the sample, the gates for the device and detectors to vary the potential of the corresponding islands, the bonding pads, the markers to align the wafer while writing the structures in the subsequent EBL step, and a continuous ground plane electrode to facilitate on-chip filtering of spurious microwave photons from reaching the sample [101]. For small structures (typical dimension $<5 \mu \mathrm{m}$ ) that will be located close to the SET junctions, a low beam current ( $1 \mathrm{nA})$ is used. 


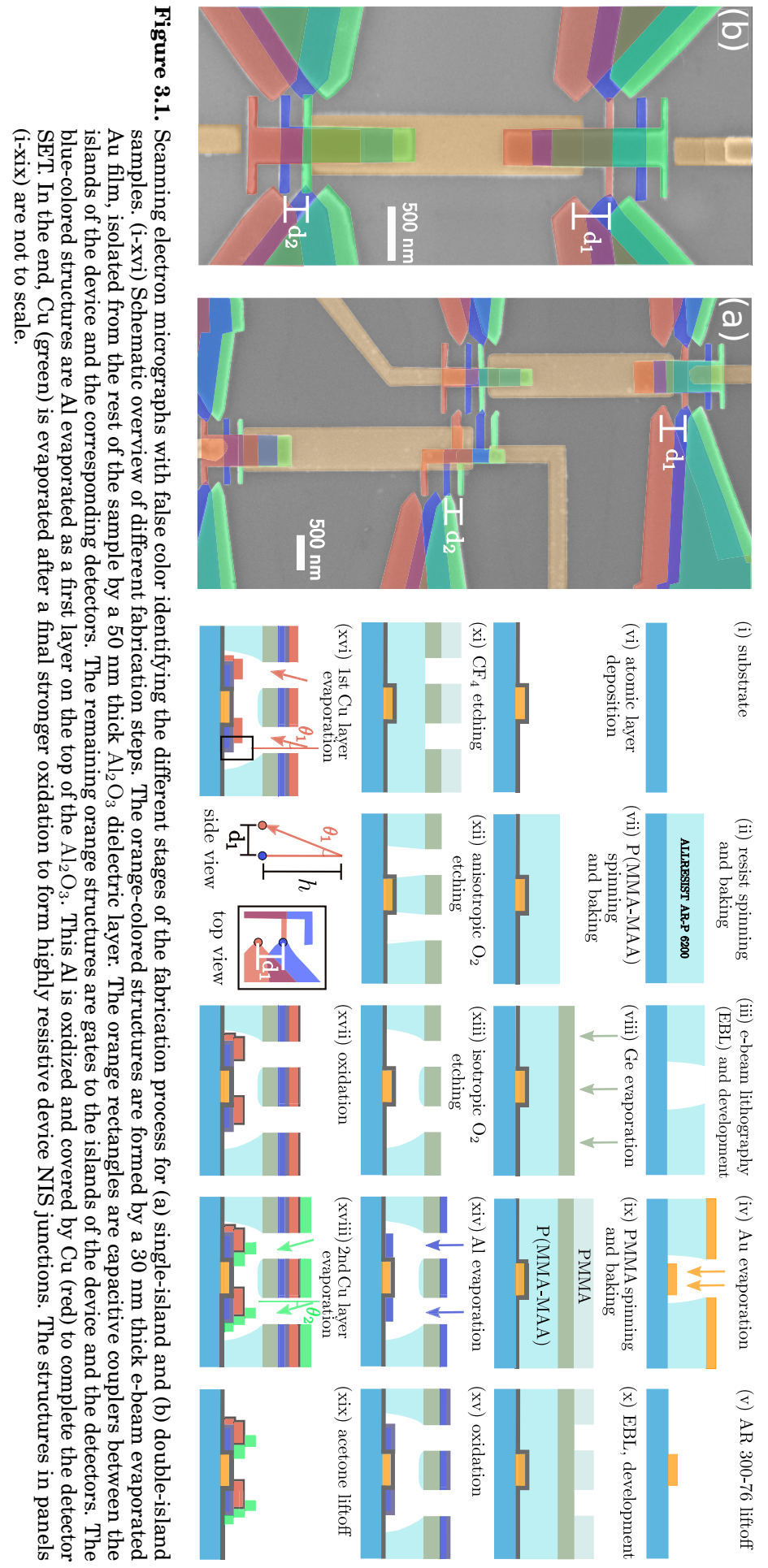


For large structures ( $>5 \mu \mathrm{m}$, usually few $100 \mu \mathrm{m}$ ) that form the pads for bonding different leads and gates, a high current (typically 100-200 nA) is used. After EBL, the exposed wafer is developed using developer AR 600-546 for $3 \mathrm{~min}$, followed by an isopropyl alcohol (IPA) rinse for $30 \mathrm{~s}$, and finally blow-dried using nitrogen, see Fig. 3.1 (iii).

The developed structures are metallized on the wafer by evaporating first $2 \mathrm{~nm}$ of Ti, then $30 \mathrm{~nm}$ of Au followed by another $2 \mathrm{~nm}$ of Ti using e-beam physical vapor deposition (typical chamber pressure in the $10^{-8}$ or $10^{-7}$ mbar range), see Fig. 3.1 (iv). The first Ti layer increases the adhesion of the gold layer to the $\mathrm{SiO}_{2}$ and the latter Ti layer improves the growth of a subsequent aluminium oxide dielectric layer.

After evaporation, the wafer is immersed in $\mathrm{AR} 300-76$ at $80^{\circ} \mathrm{C}$ for around $2 \mathrm{hr}$, followed by IPA rinse and $\mathrm{N}_{2}$ dry, to remove the remaining resist and unwanted metals from the surface. This step is called liftoff, see Fig. 3.1 (v). At the end of this step, the fabrication of the ground plane is completed.

Next, we grow an approximately $50 \mathrm{~nm}$ thick $\mathrm{Al}_{2} \mathrm{O}_{3}$ dielectric layer on the wafer, using the atomic layer deposition (ALD) technique, to isolate the ground plane from the bias leads and tunnel junction structures, see Fig. 3.1 (vi).

On the top of this ALD layer, a Ge-based hard mask [13] is prepared as follows: First, we spin-coat the wafer with approximately $400 \mathrm{~nm}$ polymethylmethacrylate (8.5)-methyl acrylic acid P(MMA-MAA) using the spinner at $5500 \mathrm{rpm}$ for $1 \mathrm{~min}$ followed by baking the wafer for $30 \mathrm{~min}$ at $180^{\circ} \mathrm{C}$, see Fig. 3.1 (vii). The next layer is $22 \mathrm{~nm} \mathrm{Ge}$, e-beam evaporated at the rate of $0.15 \AA / s$, see Fig. 3.1 (viii). The last and topmost layer is approximately $50 \mathrm{~nm}$ polymethyl methacrylate (PMMA) (molecular weight 2.2 million, $1.8 \%$ in anisole) spin-coated at $2500 \mathrm{rpm}$ for $1 \mathrm{~min}$ followed by 1 min baking at $160{ }^{\circ} \mathrm{C}$, see Fig. 3.1 (ix).

The wafer is then exposed to the electron beam for defining the final mask pattern that includes the tunnel junctions, islands, and leads (blue, green and red structures in Fig. 3.1). This step is followed by the pattern development on the topmost layer using $30 \mathrm{~s}$ immersion in a 1:3 solution (by mass) of methyl isobutyl ketone (MIBK) : IPA, IPA rinse, and subsequently drying with $\mathrm{N}_{2}$, see Fig. 3.1 (x).

After this step, the pattern is transferred to the Ge layer by reactive ion etching (RIE) with $\mathrm{CF}_{4}$ gas for 2 min and $20 \mathrm{~s}$, see Fig. 3.1 (xi). Next, the pattern is transferred to the copolymer layer during a typically $10 \mathrm{~min}$ long oxygen plasma etch using the same RIE tool, see Fig. 3.1 (xii). This phase also removes any remaining PMMA on top of the Ge film. The wafer is then exposed to a more isotropic oxygen plasma etching for $30 \mathrm{~min}$ to form a larger undercut profile in the copolymer layer. The isotropic etch is achieved by increasing the RIE chamber pressure. Fig. 3.1 (xiii) shows the side view of the mask at this stage of the sample fabrication.

Next, the final structures are deposited through the holes in the Ge 
and copolymer layers, followed by acetone liftoff. This metal deposition step consists of three-angle shadow evaporation [74] with an in-situ oxidation step between each pair of metallic layers. This is done to have an individual control over the junction transparencies, resulting in engineered resistances for the detector SETs and the device part of the sample.

As the first step of the final structure deposition, we evaporate 14 $\mathrm{nm}$ of $\mathrm{Al}$, see Fig. 3.1 (xiv). This corresponds to the blue structures in Fig. 3.1 (a, b); middle replica of the pattern at normal incidence.

After this step, the chip is exposed to 2 mbar of pure $\mathrm{O}_{2}$ for 2 min for insitu oxidation of the $\mathrm{Al}$ layer without breaking the vacuum, see Fig. 3.1 (xv). The resulting, approximately $2 \mathrm{~nm}$ thick, $\mathrm{Al}_{2} \mathrm{O}_{3}$ [102] layer creates the high-quality tunnel barrier for the NIS junctions in the detectors.

The oxidation step is followed by evaporation of $30 \mathrm{~nm}$ of $\mathrm{Cu}$ (red structures in Fig. $3.1(\mathrm{a}, \mathrm{b}))$ at an angle $\theta_{1}$ such that $\mathrm{Cu}$ from this evaporation is shifted down by $\mathrm{d}_{1}$ with respect to $\mathrm{Al}$ from the first evaporation, see Fig. 3.1 (xvi). This results in the detector tunnel junctions with an overlap area of $a_{1} \times b_{1}$. The tilt angle $\theta_{1}$ is adjusted such that the desired shift $\mathrm{d}_{1}=h \tan \left(\theta_{1}\right)$ is obtained for the mask thickness $h \approx 420 \mathrm{~nm}$.

Next, pure $\mathrm{O}_{2}$ at 5 mbar is used for 5 min to further oxidize the $\mathrm{Al}$ layer, see Fig. 3.1 (xvii). This step thickens the insulating layer for the NIS junctions in device part of the sample, and therefore increases their resistance.

In the end, $50 \mathrm{~nm}$ of $\mathrm{Cu}$ (green structures in Fig. $3.1(\mathrm{a}, \mathrm{b})$ ) is evaporated at an angle $\theta_{2}$ such that the layer is shifted up by $\mathrm{d}_{2}$ with respect to the $\mathrm{Al}$ layer, see Fig. 3.1 (xviii). This causes an overlap of area $a_{2} \times b_{2}$ between the last $\mathrm{Cu}$ layer and the first $\mathrm{Al}$ layer, and thus forms the device tunnel junctions.

The angles, $\theta_{1}$ and $\theta_{2}$, are adjusted such that the overlaps in the detector(s) are not affecting those of the device, and vice versa. After evaporation, the unwanted metals and residual mask are removed by acetone liftoff, followed by $\mathrm{N}_{2}$ dry, Fig. 3.1 (xix).

\begin{tabular}{|c|c|c|c|c|c|c|}
\hline Sample & $\theta_{1}$ & $\theta_{2}$ & $\mathrm{~d}_{1}$ & $\mathrm{~d}_{2}$ & $a_{1} \times b_{1}$ & $a_{2} \times b_{2}$ \\
\hline Single-island & $38^{\circ}$ & $-23^{\circ}$ & $340 \mathrm{~nm}$ & $210 \mathrm{~nm}$ & $25 \times 50 \mathrm{~nm}^{2}$ & $25 \times 50 \mathrm{~nm}^{2}$ \\
\hline Double-island & $36^{\circ}$ & $-21.5^{\circ}$ & $335 \mathrm{~nm}$ & $220 \mathrm{~nm}$ & $30 \times 30 \mathrm{~nm}^{2}$ & $20 \times 20 \mathrm{~nm}^{2}$ \\
\hline
\end{tabular}

Table 3.1. Fabrication parameters for the single-island and double-island samples. The shifts $\mathrm{d}_{i}$ have a typical error bar of $5 \mathrm{~nm}$ due to uncertainties in setting the tilt angles and determining the exact distances from SEM images. The junction overlap areas $a_{i} \times b_{i}$ are by design. 

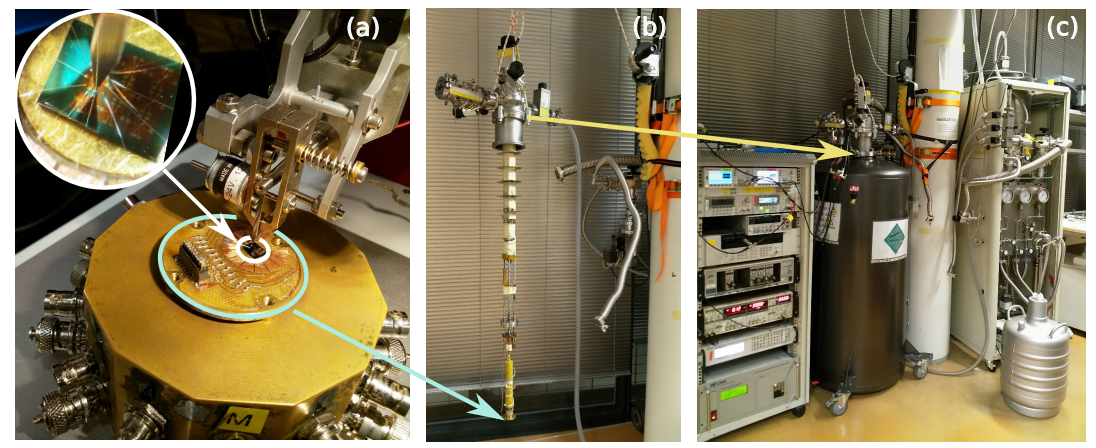

Figure 3.2. The device characterization and measurement setup. The fabricated sample with $\mathrm{Al}-\mathrm{Al}_{2} \mathrm{O}_{3}-\mathrm{Cu}$ tunnel junctions is glued with vacuum grease to the sample holder, the structure circled in turquoise in panel (a), and the sample leads are bonded to the copper pads on the sample stage. The sample stage is mounted on a platform with electrical terminals where the resistances of the tunnel junctions are checked using a digital voltmeter. Circled inset in panel (a) shows a zoomed image of the sample chip with the bonding tool of the $\mathrm{Al}$ wire bonder. The sample with desired resistances is connected to the low-temperature measurement setup, (b) which is then inserted to the dilution refrigerator He-dewar, black cylinder in (c) for cooling the sample to sub-K temperatures.

\subsection{Device characterization}

After fabrication, scanning electron micrography (SEM) of the sample is performed. This is the first test to check whether a batch of samples is good or not. Then, if they look good, their junction resistances are measured. To do so, the chip is bonded to a sample stage using a wire bonder (see Fig. 3.2 (a)), and the resistances of the sample are measured using a digital multimeter.

For samples with resistances higher than approximately $30 \mathrm{M} \Omega$, the multimeter is replaced by a room-temperature $I-V$ measurement setup, comprising of a voltage source connected to one lead of the structure and a current preamplifier to the other. The input voltage $V$ is varied and the output current $I$ from the amplifier is recorded. Next, Ohm's law $(V=I R)$ is used to infer the sample resistance $R$.

The chip, with a sample of desirable resistances, is then cooled down to the base temperature of the dilution refrigerator [103], around $50 \mathrm{mK}$, starting with measurements to infer the capacitances, charging energies and individual junction resistances.

\subsubsection{Single-electron transistor}

The $I-V$ characteristics of a single-electron transistor are measured using the setup shown in Fig. 2.3 (b) top, i.e., the blue SET. The bias voltage $V_{b}$ and gate voltage $V_{g}$ are varied to record the device output current $I$ at each point in $V_{b}$ and $V_{g}$. The resulting plot (for example Fig. 3.3 (a)) is 

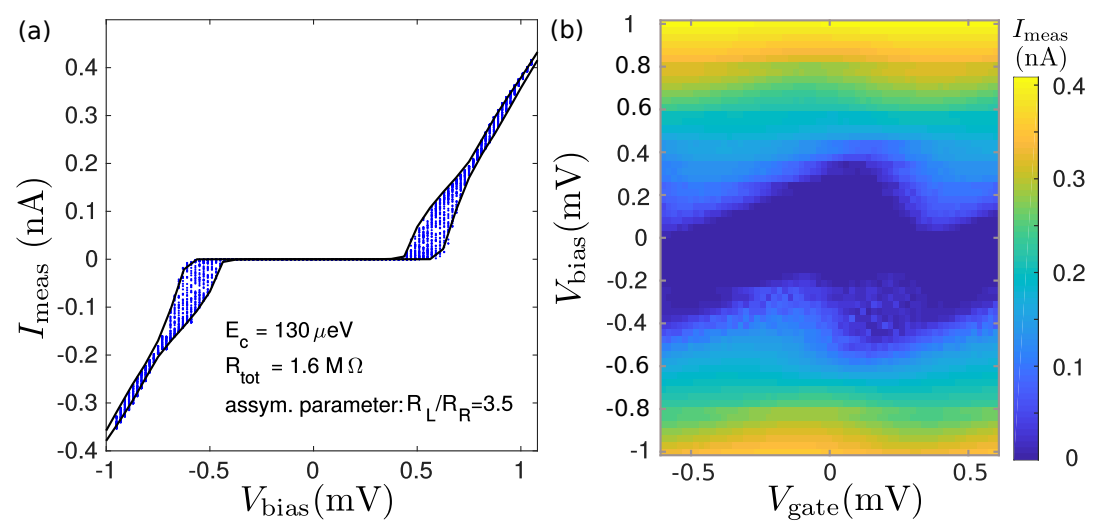

Figure 3.3. The $I-V$ characteristics of a single-electron transistor. (a) The blue dots are the measured output currents $I_{\text {meas }}$ at different bias and gate voltages. The black lines are computed using numerical simulations with parameters mentioned in the inset. (b) The color map of the SET output current with gate and bias voltages as the $\mathrm{x}$ and $\mathrm{y}$-axis, respectively.

compared with the $I-V$ characteristics obtained using standard numerical simulations based on the Fermi's golden rule and the master equation [104]. The comparison yields the sample resistance, capacitances and charging energy as the fitting parameters to the experimental curve.

Blue dots in Fig. 3.3 (a) show the output currents measured at different gate and bias voltages for an SET. The black line is obtained from the simulation, assuming a charging energy $E_{c}=130 \mu \mathrm{eV}$ and total resistance $R=1.6 \mathrm{M} \Omega$ with the asymmetry in junctions $R_{L} / R_{R}=3.5$. The asymmetry is defined as the ratio of the left junction resistance $R_{L}=1.2 \mathrm{M} \Omega$ and that of the right junction $R_{R}=0.4 \mathrm{M} \Omega$.

The SET detectors in the double-island sample had resistances of the order of $1 \mathrm{M} \Omega$ with charging energies $\approx 85 \pm 5 \mu \mathrm{eV}$. For the single-island sample, the total resistance of the device tunnel junctions was $50 \mathrm{M} \Omega$ while the detector had total resistance of $10 \mathrm{M} \Omega$. The charging energy for both SETs was approximately $140 \mu \mathrm{eV}$.

\subsubsection{Double-island structure}

To obtain the $I-V$ characteristics of the double-island structure, we modify the measurement setup shown in Fig. 2.4 (b) as follows. First, we replace the connection to ground from the right lead of the double-island by an amplifier (Femto LCA-2-10T), with amplification coefficient $10^{12} \mathrm{~V} / \mathrm{A}$, connected to a digital multimeter (Agilent 34410A). Second, we connect all the electrical terminals in both the detectors to ground. After done with these modifications to the circuit, we measure the output current, $I$, as a function of the bias voltages $V_{\mathrm{b}}$ at different gate voltages $V_{g, L}$ and $V_{g, R}$.

The high-bias $I-V$ measurement yields the total resistance of all three 


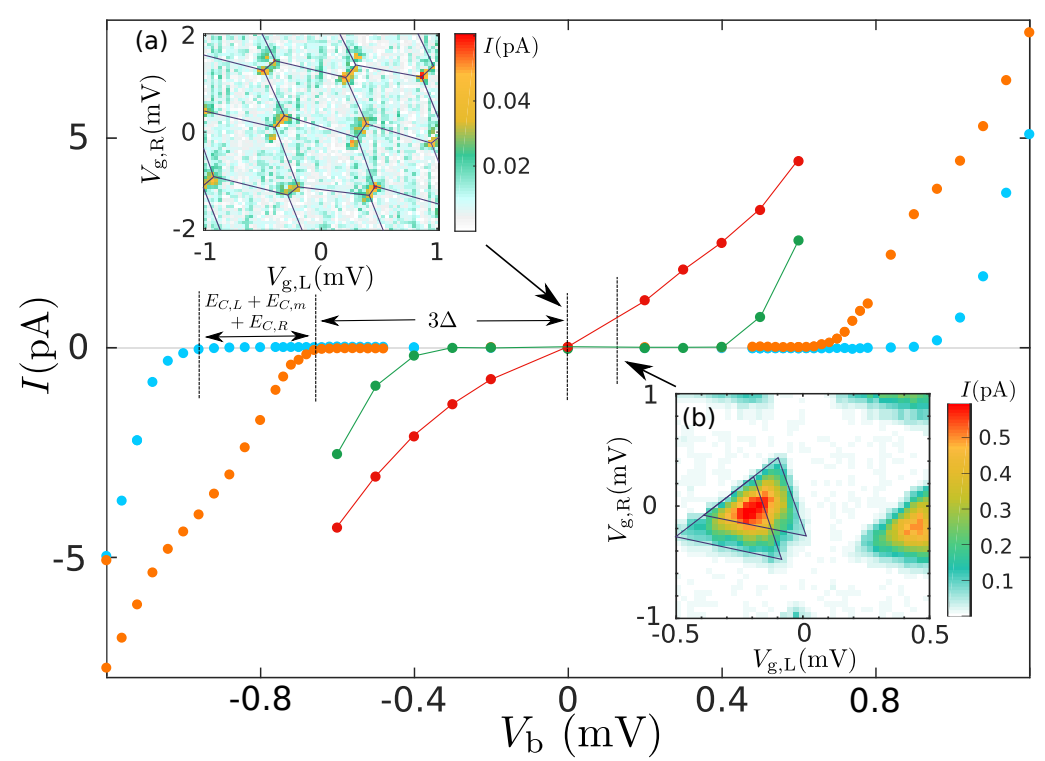

Figure 3.4. The $I-V$ characteristics and stability diagrams of the double-island. The minimum (blue) and maximum (orange) current value at a given bias voltage for the device while both the gate voltages $V_{g, L}$ and $V_{g, R}$ are varied in absence of any externally applied magnetic field. The red and green symbols show the maximum and minimum currents measured when the structure is subjected to a magnetic field, $H \approx 150 \mathrm{mT}$. At this high $H$, the superconductivity of aluminium is suppressed leading to increase in current through the doubleisland structure. The stability diagram for the bias voltages (a) $V_{\mathrm{b}}=0$ and (b) $V_{\mathrm{b}}=120 \mu \mathrm{V}$ in presence of the magnetic field. In inset (a), the honeycomb pattern typical for a double-island device [97] is visible. The high current spots correspond to the triple points at which the energies of three charge states are degenerate and the electric current can flow through the device. In inset (b), the triple points grow into triangles as the finite bias allows electrons to pass through the double-island away from degeneracy. The shape of the honeycomb structure and of the triangles allows us to determine the charging energies of the islands [97]. 
double-island junctions in series to be $R_{L}+R_{m}+R_{R} \simeq 55 \mathrm{M} \Omega$. Figure 3.4 shows the maximum (orange) and minimum (blue) values of $I$ for each bias point $V_{\mathrm{b}}$, at the base temperature $\approx 50 \mathrm{mK}$ of the dilution refrigerator. This gives the superconducting gap $\Delta=200 \mu \mathrm{eV}$ and the total charging energy of the double-island $E_{C, L}+E_{C, m}+E_{C, R}=110 \mu \mathrm{eV}$. Here, $E_{C, L}, E_{C, m}$ and $E_{C, R}$ are the charging energies of the left, middle and right junctions of the double-island, respectively.

At bias voltages $\left|V_{\mathrm{b}}\right| \lesssim 3 \Delta / e$, the direct measurement of current through the double-island structure with a room-temperature preamplifier is not possible due to the current being of the order of aA. To surpass this limitation, we restore the measurement setup in Fig. 2.4 (b) and infer the single-electron current through the structure using the output currents $I_{\mathrm{det}, \mathrm{L}}(t)$ and $I_{\mathrm{det}, \mathrm{R}}(t)$ through the detectors.

To compute the individual charging energies of the double-island junctions, we apply a magnetic field $H \approx 150 \mathrm{mT}$ to suppress the superconductivity in $\mathrm{Al}$. Then, we measure the current through the double-island at different bias voltages $V_{\mathrm{b}}$ while varying the gate voltages $V_{g, L}$ and $V_{g, R}$ to obtain the stability diagram. Insets (a) and (b) in Fig. 3.4 show the stability diagram for $V_{\mathrm{b}}=0 \mu \mathrm{V}$ and $V_{\mathrm{b}}=120 \mu \mathrm{V}$, respectively. The corresponding maximum and minimum values of $I$ for different $V_{\mathrm{b}}$ are presented in the main figure by the red and green symbols, respectively. Comparing the stability diagrams (a) and (b) to the theory from [97] we extract the charging energies $E_{C, L}=60 \mu \mathrm{eV}, E_{C, m}=10 \mu \mathrm{eV}$, and $E_{C, R}=40 \mu \mathrm{eV}$.

\subsection{Measurement setup}

The schematic sketch of the measurement setup for both a single-island and a double-island structure is presented in Chapter 2. The measurements presented in this thesis are performed at the base temperature of the dilution refrigerator, close to $40 \mathrm{mK}$, and slightly elevated temperatures up to $250 \mathrm{mK}$. For details on the refrigeration techniques, refer to [75].

The chips with either a single-island or a double-island sample were enclosed in a sample holder (marked by the circle in Fig. 3.2 (a)) with 12 measurement lines for measuring current and changing bias or gate voltages. The sample stage is then connected to the measurement setup, an example of which is shown in Fig. 3.2 (b). It shows the insert of a plastic dilution refrigerator [103] which is to be dipped in the He-dewar, black cylinder in Fig. 3.2 (c), to maintain the sample at $\mathrm{mK}$ temperatures. All the signal lines connecting the sample to the outer measurement setup are filtered by Thermocoax cables [105], and the sample stage is thermally anchored to the mixing chamber.

The measurement data from the experiments on single-island (double- 
island) were acquired at the sampling frequency of $50 \mathrm{kHz}(25 \mathrm{kHz})$, which is much higher than the transition rates of electron onto and out of the device island, $\approx 100 \mathrm{~Hz}$. The transition rates in detector island are $\approx$ $\mathrm{GHz}$ with a detector bandwidth of $10 \mathrm{kHz}$ given by the output current rise time. The superconductivity in the experiments is used as an appliance to obtain slow electron transitions onto and out of the device island such that these transitions can be followed by the detectors. All the measurements apart from the ones for computing the double dot capacitance and charging energy (mentioned in Figs. 2.6 and 3.4) were performed in the superconducting state of $\mathrm{Al}$ (the superconductor used or NIS junctions in this thesis). 
Experimental methods 


\section{Current distribution of a bistable system}

A bistable system is one of the simplest dynamical systems. This bistability is very common in nature [106], e.g., in solid state [107-109], magnetic [110-112], and biological systems [113]. In an electronic system (such as SETs in Sec. 2.3), this bistability could be pictured as the jump between two stable states in the system. However, it is not always easy to compute these transition rates. For example, when the detector (used to witness the system jumps) response time is larger than the time needed for system to jump. In Publication I, we propose a new approach to determine the rates that works even in such case.

The current through a bistable system has a bimodal distribution. However, when averaged over long time this distribution takes the form of an ellipse [5, 106]. It is interesting to investigate this crossover from bimodal to elliptic behavior. This is the focus of Publication II. This chapter provides the experimental results of Publications I and II, and the required theoretical background.

We start with a general discussion of the current output from a bistable system, which includes the telegraph signal and the white noise. Next, the calculation of the distribution of the fluctuating current, filtered in two different ways, is presented in brief. In Sec. 4.2 , a procedure to compute the transition rates between the system states using the distribution of filtered current is presented. The chapter ends with theoretical results on the properties of time-averaged current distribution in a general bistable system, and its comparison to the experimental data from the experiments on single-island structure, see Sec. 2.3 and Publication II.

\subsection{Output current}

The output current through the detector coupled to a bistable system (for example the blue SET in Fig. 2.3) is presented by

$$
I_{d}(t)=I_{\text {tel }}(t)+\xi(t) .
$$



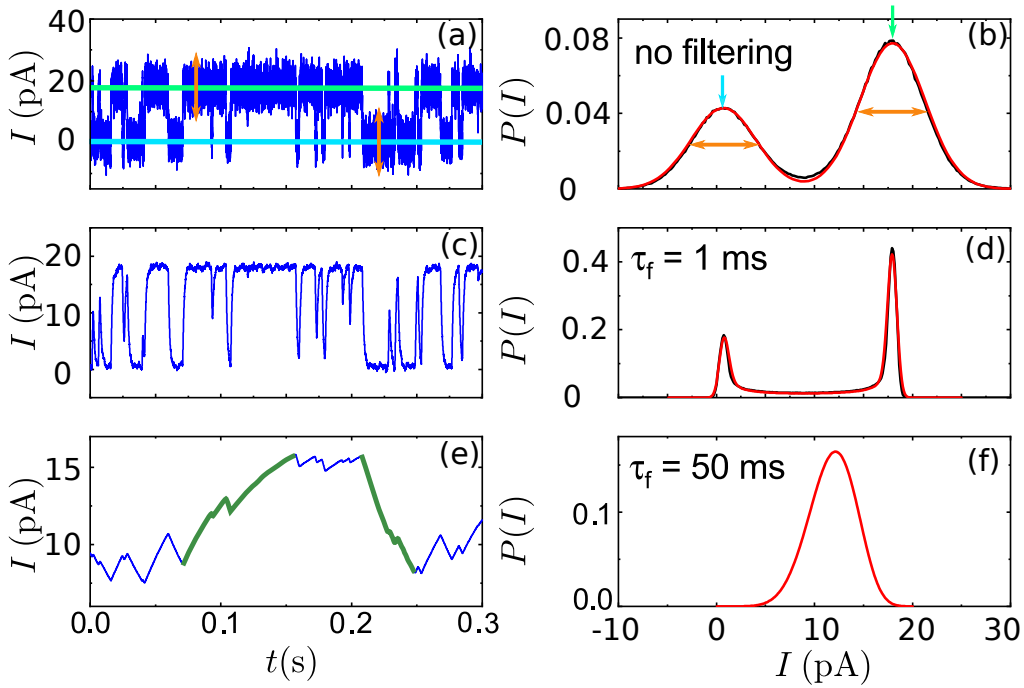

Figure 4.1. (a) Sample time trace and (b) the corresponding distribution of the output current through a detector coupled to an SEB (see Fig. 2.3 for the measurement setup). The filtered signal (4.3) and the corresponding distribution plotted in panels (c) and (d), respectively, for $\tau_{f}=1 \mathrm{~ms}$. (e) and (f) are the filtered signal and distribution with $\tau_{f}=50 \mathrm{~ms}$. Adapted from Publication I.

Here, the first term on the right hand side $I_{\text {tel }}(t)$ is the telegraph signal, which shows the jumps between the system states and the relaxation after a jump has occurred. The second term, $\xi(t)$, incorporates the noise in the detector output current caused by different sources, e.g., white noise, $1 / f$ noise, and $50 \mathrm{~Hz}$ noise due to pick-up from the power lines.

Figure 4.1 (a) shows a typical detector output current from a bistable system. The cyan and green lines mark the two possible system states, namely 0 and 1 extra electrons on the island of the system (e.g., the turquoise island in Fig. 2.3), respectively. The variation of these states over time is represented by $I_{\text {tel }}(t)$. The spreading shown in orange arrows is due to the noise in output signal, $\xi(t)$. Figure 4.1 (b) shows the corresponding distribution of the current with two peaks, one corresponding to each state.

Next, we convolve the output signal, $I_{d}(t)$, with an arbitrary function $f(t)$ to obtain $I(t)$ as

$$
I(t)=\int_{-\infty}^{\infty} d t^{\prime} f\left(t-t^{\prime}\right) I_{d}\left(t^{\prime}\right) .
$$

We use different functional forms of $f(t)$ to serve our purpose. For example, to obtain the time-averaged current (so called "running average") over time $\tau_{f}$ from initial time $t-\tau_{f}$, we use $f(t)=\theta(t) \theta\left(-t+\tau_{f}\right) / \tau_{f}$. Here, $\theta(x)$ is the Heaviside step function

$$
\theta(x)= \begin{cases}0, & x<0 \\ 1, & x>0\end{cases}
$$


In order to apply a low pass filtering to the signal $I_{d}(t)$ with a bandwidth of $1 / \tau_{f}$, we use $f(t)=e^{-t / \tau_{f}} \theta(t) / \tau_{f}$.

In the following sections, we consider both the aforementioned convolution functions for two different configurations. First, we study a case in which $f(t)$ is a filtering function with no influence on detector response time $\tau_{f}$. Second, we assume a fast responding detector with function $f(t)$ to define the time-averaging of the detector output current $I_{d}(t)$.

\subsection{Calculation of the transition rates using low pass filtering}

In this section, we present a way to compute the transition rates of a bistable system using the cumulants of the net current distribution. We apply low pass filtering to the output signal $I_{d}(t)$, i.e., the convolved-current $I(t)$ in Eq. (4.2) takes the form

$$
I(t)=\int_{-\infty}^{\infty} d t^{\prime} e^{-\left(t-t^{\prime}\right) / \tau_{f}} \theta\left(t-t^{\prime}\right) / \tau_{f} I_{d}\left(t^{\prime}\right) .
$$

The probability distribution of the resulting current is then computed and the cumulants of the distribution are used to compute the transition rates between the system states. The time convolution (4.3) models a detector with the bandwidth $1 / \tau_{f}$. Our goal is to show that the rates can be determined even if the detector is quite slow.

Figures 4.1 (c) and (e) show the convolved current, $I$, for the sample traces shown in Fig. 4.1 (a). The panels (d) and (f) illustrate the corresponding distributions. We now present a framework to compute the probability distribution of the output current for any bistable system.

\subsubsection{Probability distribution}

We introduce $\mathcal{P}_{i}(I, t)$ as the probability density distribution of the current corresponding to system state $i$. Considering vanishing noise $\xi(t)=0$, the probability of system to be in state $i$ is defined as

$$
p_{i}=\int_{I_{0}}^{I_{1}} \mathcal{P}_{i}(I, t) d I .
$$

Here, the current $I_{1}\left(I_{0}\right)$ corresponding to system state $1(0)$ is the upper (lower) bound of the integral as it is the maximum (minimum) value current can acquire in the absence of any noise. The transition rate from state 1 to 0 is denoted as $\gamma_{\downarrow}$ and from 0 to 1 as $\gamma_{\uparrow}$, with $\gamma_{\Sigma}=\gamma_{\uparrow}+\gamma_{\downarrow}$.

The time evolution of distribution $\mathcal{P}_{i}(I, t)$ is given by the theory of stochastic jump processes $[96,114]$. The evolution equations of $\mathcal{P}_{0}$ and $\mathcal{P}_{1}$ read

$$
\begin{aligned}
& \partial_{t} \mathcal{P}_{0}(I, t)=\tau_{f}^{-1} \partial_{I}\left[\left(I-I_{0}\right) \mathcal{P}_{0}(I, t)\right]-\gamma_{\uparrow} \mathcal{P}_{0}(I, t)+\gamma_{\downarrow} \mathcal{P}_{1}(I, t), \\
& \partial_{t} \mathcal{P}_{1}(I, t)=\tau_{f}^{-1} \partial_{I}\left[\left(I-I_{1}\right) \mathcal{P}_{1}(I, t)\right]+\gamma_{\uparrow} \mathcal{P}_{0}(I, t)-\gamma_{\downarrow} \mathcal{P}_{1}(I, t) .
\end{aligned}
$$


The first term, $\tau_{f}^{-1} \partial_{I}\left[\left(I-I_{i}\right) \mathcal{P}_{i}(I, t)\right]$, captures the relaxation time of the system after a jump between the states has occurred, for example see the green line in Fig. 4.1 (e). They account for the continuity of current. The next two terms capture the change in probability $\mathcal{P}_{i}(I, t)$ due to the system jumping from $i$ to another state or from some other state to $i$. It accounts for the discrete jumps in the system state. The first term becomes negligible in the case detector response is very fast, $\gamma_{\Sigma} \tau_{f} \ll 1$. For situations where the detector response is slower than the system transition rate, i.e., $\gamma_{\Sigma} \tau_{f} \gtrsim 1$, the first term becomes significant and cannot be ignored.

At steady state, $\partial_{t} \mathcal{P}_{i}(I, t)=0$. This results in the time-independent distributions

$$
\mathcal{P}_{0}(I)=\frac{I_{1}-I}{I_{1}-I_{0}} \mathcal{P}(I), \quad \mathcal{P}_{1}(I)=\frac{I-I_{0}}{I_{1}-I_{0}} \mathcal{P}(I),
$$

with $\mathcal{P}(I)=\mathcal{P}_{0}(I)+\mathcal{P}_{1}(I)$. Using the time evolution equation (4.4), we obtain the stationary distribution of $I$ as

$$
\mathcal{P}(I)=\frac{\Gamma\left(\gamma_{\Sigma} \tau_{f}\right)}{\Gamma\left(\gamma_{\uparrow} \tau_{f}\right) \Gamma\left(\gamma_{\downarrow} \tau_{f}\right)} \frac{\left(I-I_{0}\right)^{\gamma_{\uparrow} \tau_{f}-1}\left(I_{1}-I\right)^{\gamma_{\downarrow} \tau_{f}-1}}{\left(I_{1}-I_{0}\right)^{\gamma_{\Sigma} \tau_{f}-1}},
$$

where $\Gamma(x)$ is the gamma function. This functional form is well known in statistics as the Beta distribution. To this distribution, we now convolve the distribution of filtered noise, $W_{\xi}(I)=\left\langle\delta\left(I-\frac{1}{\tau_{f}} \int_{-\infty}^{t} d t^{\prime} e^{-\left(t-t^{\prime}\right) / \tau_{f}} \xi\left(t^{\prime}\right)\right)\right\rangle$, in order to incorporate the influence of noise on the distribution (4.6), namely

$$
P(I)=\int_{I_{0}}^{I_{1}} d I^{\prime} W_{\xi}\left(I-I^{\prime}\right) \mathcal{P}\left(I^{\prime}\right) .
$$

This resulting distribution has now the information of both the telegraph signal and the noise introduced due to the measurement.

In general, when the detector is faster than the transitions in the system, the distribution of measured current for a bistable system has a bimodal peak [Fig. 4.1 (b) and blue line in inset of Fig. 4.2 (a)]. In the case of slow detector [Fig. 4.1 (f) and red line in inset of Fig. 4.2 (a)], the two peaks merge and it is hard to resolve the two possible states of the system and hence the relevant transition rates using the traditional method [115] (see Sec. 4.2.3) is hard to compute.

Next, we present the use of distribution (4.7) to calculate the system transition rates in such cases. In Fig. 4.2, the efficiency of this method is demonstrated by analyzing the output currents $I_{d}$ from two samples: sample A (blue lines) where $\gamma_{\Sigma} \tau_{f} \ll 1$ and traditional method can be used to compute its rates, and sample B (red lines) where $\gamma_{\Sigma} \tau_{f} \gg 1$ and traditional method can no longer be used to compute its rates. 

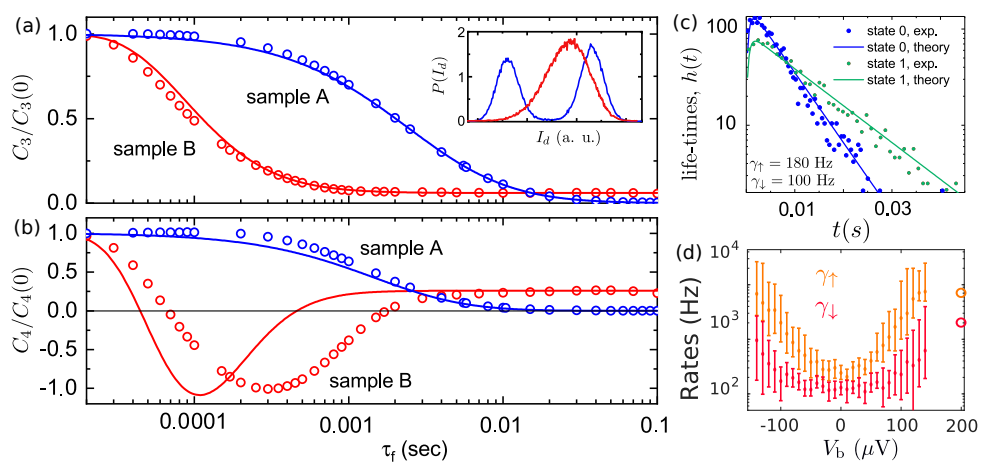

Figure 4.2. The cumulants, (a) $C_{3}$ and (b) $C_{4}$, from experiments on sample A (blue symbols) and B (red symbols) and theory curves (solid lines), Eqs. (4.10), with rates obtained by fitting these equations to the experimental data. Inset: the probability distribution of current $I_{d}$ from sample A (blue), two peaks corresponding to two system states are clearly visible, and sample B (red) at bias voltage $V_{b}=200 \mu \mathrm{V}$, one peak, hard to identify the two states. (c) Life-times distribution for system states 0 (blue) and 1 (green) for sample A. (d) The rates $\gamma_{\uparrow}$ (orange dots) and $\gamma_{\downarrow}$ (red dots) calculated using traditional threshold method for sample $B$ at low bias values. The open circles are rates calculated using fit to the cumulants (4.10) at $V_{b}=200 \mu \mathrm{V}$.

\subsubsection{Transition rates}

Using the distribution (4.7), we calculate the cumulants $\mathcal{C}_{i}$ of the output current. The first cumulant gives the average current as

$$
\mathcal{C}_{1}\left(\tau_{f}\right)=\langle I\rangle=\left(\gamma_{\downarrow} I_{0}+\gamma_{\uparrow} I_{1}\right) / \gamma_{\Sigma},
$$

which is independent of time $\tau_{f}$. The second cumulant $\mathcal{C}_{2}=\left\langle(I-\langle I\rangle)^{2}\right\rangle$ gives the variation of the detector output current $I_{d}$ as

$$
\mathcal{C}_{2}\left(\tau_{f}\right)=\frac{\gamma_{\uparrow} \gamma_{\downarrow}\left(I_{1}-I_{0}\right)^{2}}{\gamma_{\Sigma}^{2}\left(1+\gamma_{\Sigma} \tau_{f}\right)}+\sigma_{\xi}^{2}\left(\tau_{f}\right)
$$

Here, the first term is the variation of telegraph noise and the second term $\sigma_{\xi}^{2}\left(\tau_{f}\right)$ is the variance of filtered noise, $W_{\xi}(I)$. This incorporates the noise in our measured current. Thus, this is a relevant quantity to look at if we are interested in understanding the noise component of our signal. As our present focus is to infer the rates, we look for higher order cumulants, which might depend on system transition rates.

The third and the fourth cumulants, skewness and kurtosis, respectively, $\mathcal{C}_{3}=\left\langle(I-\langle I\rangle)^{3}\right\rangle$ and $\mathcal{C}_{4}=\left\langle(I-\langle I\rangle)^{4}\right\rangle-3 \mathcal{C}_{2}^{2}$ normalized to their values at $\tau_{f}=0$ are given by

$$
\begin{aligned}
& \frac{\mathcal{C}_{3}\left(\tau_{f}\right)}{\mathcal{C}_{3}(0)}=\frac{2}{\left(1+\gamma_{\Sigma} \tau_{f}\right)\left(2+\gamma_{\Sigma} \tau_{f}\right)}+\frac{\mathcal{C}_{3, \infty}}{\mathcal{C}_{3}(0)} \\
& \frac{\mathcal{C}_{4}\left(\tau_{f}\right)}{\mathcal{C}_{4}(0)}=\frac{6\left[\left(\gamma_{\uparrow}-\gamma_{\downarrow}\right)^{2}\left(1+\gamma_{\Sigma} \tau_{f}\right)-\gamma_{\uparrow} \gamma_{\downarrow}\left(2+\gamma_{\Sigma} \tau_{f}\right)\right]}{\left(1+\gamma_{\Sigma} \tau_{f}\right)^{2}\left(2+\gamma_{\Sigma} \tau_{f}\right)\left(3+\gamma_{\Sigma} \tau_{f}\right)\left[\gamma_{\uparrow}^{2}+\gamma_{\downarrow}^{2}-4 \gamma_{\uparrow} \gamma_{\downarrow}\right]}+\frac{\mathcal{C}_{4, \infty}}{\mathcal{C}_{4}(0)} .
\end{aligned}
$$


They are not influenced by the Gaussian white noise. However, the effect of non-Gaussian $1 / f$ noise is captured by the long-time limit of the cumulants, i.e., $\mathcal{C}_{3, \infty}$ and $\mathcal{C}_{4, \infty}$, which depends weakly on $\tau_{f}$.

Since, the first terms in cumulants, $\mathcal{C}_{3}$ and $\mathcal{C}_{4}$ are dependent only on system parameters, $\gamma_{\uparrow}$ and $\gamma_{\downarrow}$, and detector bandwidth $\tau_{f}^{-1}$, they can be jointly used to extract the rates $\gamma_{\uparrow}$ and $\gamma_{\downarrow}$ by fitting the dependence of the cumulants on $\tau_{f}$. Note that, this method of computing the rates does not assume anything about the distribution of output current. That is, it works equally well for simpler case in which the detectors are fast.

\subsubsection{Experimental test of the cumulant method}

Figure 4.2 (a) and (b) show the cumulants $\mathcal{C}_{3}$ and $\mathcal{C}_{4}$ obtained using experimental data (symbols) from samples A (blue) and B (red), along with the theory fits (4.10) (solid lines) with $\gamma \mathrm{s}$ computed by fitting Eqs. (4.10) to the experimental plot. The samples A and B are single-island structures with measurement setup similar to one in Fig. 2.3 (b). The transition rates in sample $\mathrm{B}$ are made faster by increasing the bias voltage $V_{\mathrm{b}}$ such that detector is not able to follow the transitions in the SET coupled to it, i.e., $\gamma_{\Sigma} \tau_{f} \gg 1$.

As a test of the calculated rates, we also computed the rates for sample A using the traditional method. This involves fitting the experimental distribution of life-times $h(t)$ of states to the ones expected from theory given by

$$
h_{0,1}=\frac{2 \gamma_{\uparrow, \downarrow} \gamma_{\operatorname{det}} e^{-\lambda t / 2} \sinh \left(\sqrt{\lambda^{2}-4 \gamma_{\uparrow, \downarrow} \gamma_{\operatorname{det}}} t / 2\right)}{\sqrt{\lambda^{2}-4 \gamma_{\uparrow, \downarrow} \gamma_{\operatorname{det}}}} .
$$

Here the effective detector bandwidth $\gamma_{\text {det }}=\left(\tau_{f} \ln 2\right)^{-1}=1443 \mathrm{~Hz}$ with $\tau_{f}=1 \mathrm{~ms}$ and $\lambda=\gamma_{\Sigma}+\gamma_{\text {det }}$. Figure 4.2 (c) shows the theory fit Eqs. (4.11) (line) to the experimental distribution (symbol) for states 0 (blue) and 1 (green). The rates computed using the cumulants are in agreement with the ones from traditional method.

For sample B, the calculation using traditional methods is not possible. To check the results, we compute the transition rates for lower bias voltages (dots in Fig. 4.2 (d) with experimental error bars) and compare the extrapolated values from lower bias rates to the ones obtained for $200 \mu \mathrm{V}$ bias using the cumulants. We find the extrapolated rates to be in agreement with the rates obtained using cumulants.

\subsection{Distribution of time-averaged currents}

In this section, we present a framework for the calculation of probability distribution of time-averaged current through a bistable system, and compare it with the experimental results. The distribution has system specific 
properties and can be used to understand the fluctuations in an arbitrary bistable system coupled to the measurement setup.

The section starts with the definition of time-averaged current and proceeds towards the calculation of distribution. Lastly, we discuss the short and long averaging-time limit of the distribution and a fluctuation relation that holds for the distribution.

We consider the time-averaging function, $f(t)=\theta(t) \theta(-t+\tau) / \tau$ with averaging-time $\tau$, to convolve with the output signal $I_{d}$. Consequently, Eq. (4.3) can be used to define the time-averaged current $I_{\tau}(t)$ as

$$
I(\tau, t)=\int_{-\infty}^{-\infty} d t^{\prime} \frac{\theta\left(t-t^{\prime}\right) \theta\left(-t+t^{\prime}+\tau\right)}{\tau} I_{d}\left(t^{\prime}\right)=\int_{t-\tau}^{t} d t^{\prime} \frac{I_{d}\left(t^{\prime}\right)}{\tau} .
$$

Following the representation (4.1) for detector output current, we re-write the time-averaged current as

$$
I(\tau, t)=[1-\mathcal{N}(\tau, t)] I_{0}+\mathcal{N}(\tau, t) I_{1}+\xi_{\tau},
$$

where $\xi_{\tau}$ is the time-averaged noise and the first two terms correspond to the time-averaging of the telegraph signal $I_{\text {tel }}(t)$. The currents $I_{0}$ and $I_{1}$ denote the telegraph signal $I_{\text {tel }}(t)$ corresponding to 0 and 1 extra electrons on the device island, respectively. For simplicity, we define the time trace $N(t)$ corresponding to the absence or presence of an extra electron on the island of the system as rescaled $I_{\text {tel }}(t)$, such that

$$
N(t)= \begin{cases}1 & \text { if } I_{\text {tel }}(t)=I_{1} \\ 0 & \text { if } I_{\text {tel }}(t)=I_{0}\end{cases}
$$

Consequently, the time-averaged number of extra electrons on the island [116] is given by

$$
\mathcal{N}(\tau, t)=\frac{1}{\tau} \int_{t-\tau}^{t} d t^{\prime} N\left(t^{\prime}\right)
$$

and the rescaled average current (4.13) becomes

$$
\mathcal{I}=\frac{I(\tau, t)-I_{0}}{I_{1}-I_{0}}=\mathcal{N}(\tau, t)+\tilde{\xi}_{\tau} .
$$

Here the last term $\tilde{\xi}_{\tau}=\xi_{\tau} /\left(I_{1}-I_{0}\right)$ is the rescaled time-averaged noise.

\subsubsection{Steady-state probability of states}

For simplicity, we consider a configuration in which, the detector response time is faster than the transitions between states, i.e., $\gamma_{\Sigma} \tau_{f} \ll 1$. In this case, integration of the evolution equation of the probability distribution $\mathcal{P}_{i}$, Eq. (4.4), over the currents $I_{0}$ and $I_{1}$ gives us the time evolution equation for probability $p_{i}$ corresponding to state $i$ as (2.19)

$$
\begin{aligned}
& \partial_{t} p_{0}=-\gamma_{\uparrow} p_{0}+\gamma_{\downarrow} p_{1}, \\
& \partial_{t} p_{1}=\gamma_{\uparrow} p_{0}-\gamma_{\downarrow} p_{1} .
\end{aligned}
$$


At steady state, there is no net change in probability and therefore,

$$
p_{1}=\frac{\gamma_{\uparrow}}{\gamma_{\Sigma}} \text { and } p_{0}=\frac{\gamma_{\downarrow}}{\gamma_{\Sigma}},
$$

where the total transition rate $\gamma_{\Sigma}=\gamma_{\uparrow}+\gamma_{\downarrow}$ and we have assumed that only 0 or 1 extra electron on the island is allowed, i.e., $p_{0}+p_{1}=1$.

\subsubsection{Probability distribution}

The probability distribution $\mathcal{P}(\mathcal{N}, \tau)$ of the trace $N(t)$ averaged over time $\tau$ can be defined in terms of the generating function $F(\lambda)$ as

$$
\mathcal{P}(\mathcal{N}, \tau)=\int \frac{d \lambda}{2 \pi} e^{-i \lambda \mathcal{N}} F(\lambda),
$$

where $F(\lambda)=\left\langle e^{i \lambda \mathcal{N}}\right\rangle$. Following Bagrets and Nazarov [117], it can be expressed as

$$
F(\lambda)=e^{T} \boldsymbol{\Lambda}(\tau, \lambda) \boldsymbol{P}(0),
$$

where

$$
\boldsymbol{e}^{T}=(1,1), \quad \boldsymbol{\Lambda}(\tau, \lambda)=\exp \left(\left[\begin{array}{cc}
-\gamma_{\uparrow} & u \gamma_{\downarrow} \\
u \gamma_{\uparrow} & -\gamma_{\downarrow}+i \lambda / \tau
\end{array}\right] \tau\right), \quad \boldsymbol{P}(0)=\left(\begin{array}{c}
p_{0}(0) \\
p_{1}(0)
\end{array}\right) .
$$

Here, we have introduced a parameter $u=1$ such that after Taylor expansion, the term $\propto u^{m}$ gives the contribution of $m$ jumps. Using the generating function $F(\lambda)$ in Eq. (4.20), we obtain the distribution

$$
\mathcal{P}(\mathcal{N}, \tau)=\sum_{m=0}^{\infty} P_{m}(\tau, \mathcal{N})
$$

where the distribution $P_{m}(\tau, \mathcal{N})$ gives the contribution of trajectories with $m$ jumps. For no jump, i.e., $m=0$, the distribution reads

$$
P_{0}(\tau, \mathcal{N})=\frac{\gamma_{\uparrow}}{\gamma_{\Sigma}} e^{-\gamma_{\downarrow \tau}} \delta(\mathcal{N}-1)+\frac{\gamma_{\downarrow}}{\gamma_{\Sigma}} e^{-\gamma_{\uparrow \tau}} \delta(\mathcal{N}) .
$$

For even $m$, the distribution is given by

$$
\begin{aligned}
P_{m}(\tau, \mathcal{N})= & 2 \theta[\mathcal{N}(1-\mathcal{N})] e^{-\frac{\gamma_{\Sigma} \tau}{2}} e^{\left(\gamma_{\uparrow}-\gamma_{\downarrow}\right)\left(\mathcal{N}-\frac{1}{2}\right) \tau} \\
& \times\left(\frac{\gamma_{\uparrow}}{\gamma_{\Sigma}} \mathcal{N}+\frac{\gamma_{\downarrow}}{\gamma_{\Sigma}}(1-\mathcal{N})\right) \frac{\left(\gamma_{\uparrow} \gamma_{\downarrow} \tau^{2}\right)^{\frac{m}{2}}}{m} \frac{\mathcal{N}^{\frac{m-2}{2}}(1-\mathcal{N})^{\frac{m-2}{2}}}{\left[\left(\frac{m-2}{2}\right) !\right]^{2}},
\end{aligned}
$$

and for odd $m$, the distribution takes the form

$$
\begin{aligned}
P_{m}(\tau, \mathcal{N})=2 \theta[\mathcal{N}(1-\mathcal{N})] e^{-\frac{\gamma_{\Sigma} \tau}{2}} e^{\left(\gamma_{\uparrow}-\gamma_{\downarrow}\right)\left(\mathcal{N}-\frac{1}{2}\right) \tau} \\
\times \frac{\sqrt{\gamma_{\uparrow} \gamma_{\downarrow}}}{\gamma_{\Sigma}}\left(\gamma_{\uparrow} \gamma_{\downarrow} \tau^{2}\right)^{\frac{m}{2}} \frac{\mathcal{N}^{\frac{m-1}{2}}(1-\mathcal{N})^{\frac{m-1}{2}}}{\left[\left(\frac{m-1}{2}\right) !\right]^{2}}
\end{aligned}
$$


Combining the distribution of different number of jumps, we obtain the total distribution [see Publication II and Ref. [118] for details]

$$
\begin{aligned}
\mathcal{P}(\mathcal{N}, \tau)= & \frac{\gamma_{\uparrow}}{\gamma_{\Sigma}} e^{-\gamma_{\downarrow} \tau} \delta(\mathcal{N}-1)+\frac{\gamma_{\downarrow}}{\gamma_{\Sigma}} e^{-\gamma_{\uparrow} \tau} \delta(\mathcal{N}) \\
+ & \theta[\mathcal{N}(1-\mathcal{N})] e^{-\frac{\gamma_{\Sigma} \tau}{2}} e^{\left(\gamma_{\uparrow}-\gamma_{\downarrow}\right)\left(\mathcal{N}-\frac{1}{2}\right) \tau}\left[\left(1+2 \frac{\gamma_{\uparrow}-\gamma_{\downarrow}}{\gamma_{\Sigma}}\left(\mathcal{N}-\frac{1}{2}\right)\right)\right. \\
& \left.\times \frac{I_{1}\left(2 \sqrt{\gamma_{\uparrow} \gamma_{\downarrow} \mathcal{N}(1-\mathcal{N})} \tau\right)}{\sqrt{\gamma_{\uparrow} \gamma_{\downarrow} \mathcal{N}(1-\mathcal{N})}}+\frac{4}{\gamma_{\Sigma}} I_{0}\left(2 \sqrt{\gamma_{\uparrow} \gamma_{\downarrow} \mathcal{N}(1-\mathcal{N}) \tau}\right)\right] \frac{\gamma_{\uparrow} \gamma_{\downarrow} \tau}{2} .
\end{aligned}
$$

This expression shows the distribution of time-averaged system state for any time $\tau$. We incorporate the effect of noise as done for filteredcurrent distribution $\mathcal{P}(I)$ by using Eq. (4.7). This allows us to obtain the distribution for rescaled time-average current $\mathcal{I}$ [defined in Eq. (4.16)] as

$$
\mathcal{P}(\mathcal{I}, \tau)=\int_{0}^{1} d \mathcal{N}^{\prime} W_{\xi}\left(\mathcal{N}-\mathcal{N}^{\prime}\right) \mathcal{P}\left(\mathcal{N}^{\prime}, \tau\right)
$$

Here, $W_{\xi}\left(\mathcal{N}-\mathcal{N}^{\prime}\right)$ is the distribution of noise in the detector output current [see Publication II for details]. We discuss below the resulting distribution in short and long-time limits.

\subsubsection{Short-time limit}
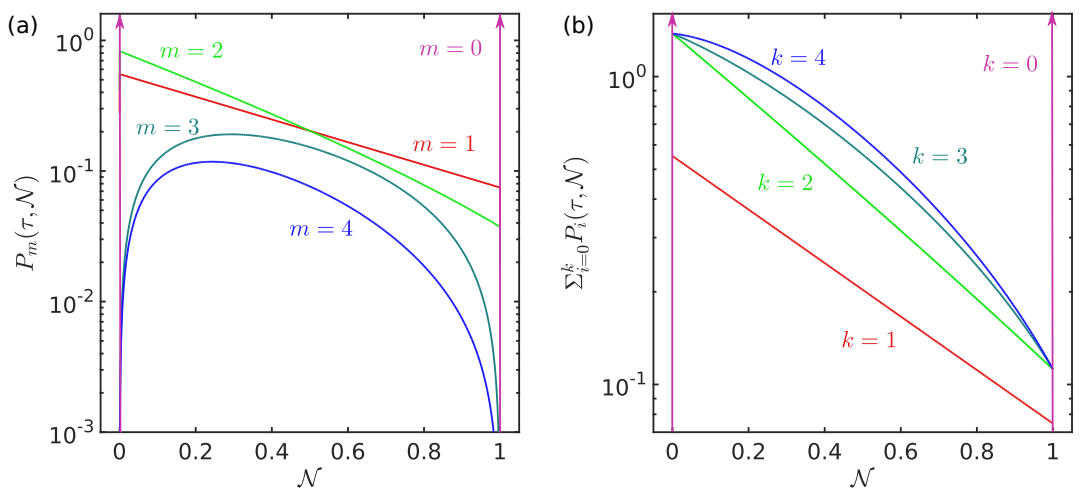

Figure 4.3. (a) The probability distribution $P_{m}(\tau, \mathcal{N})$ presents the contribution of trajectories with $m$ jumps. (b) The sum $\sum_{m=0}^{k} P_{m}(\tau, \mathcal{N})$ considering $k$ the number of total jumps during the averaging-time $\tau=10 \mathrm{~ms}$. The plot is generated for a system with the transition rates $\gamma_{\uparrow}=100 \mathrm{~Hz}$ and $\gamma_{\downarrow}=300 \mathrm{~Hz}$.

For very small averaging-time $\tau$, there is a possibility that only few or no jump have occurred in the system, during the time $\tau$. If there is no jump, $P_{0}(\tau, \mathcal{N})$ describes the probability distribution corresponding to the initial state of the system. Similarly, if there is only few jumps in the system during the time $\tau$, individual jumps have a significant contribution to the 
probability distribution. The contributions of the first four jumps to the total time-averaged distribution $\mathcal{P}(\mathcal{N}, \tau)$ are given by

$$
\begin{gathered}
P_{1}(\tau, \mathcal{N})=2 \frac{\gamma_{\uparrow} \gamma_{\downarrow} \tau}{\gamma_{\Sigma}} e^{-\frac{\gamma_{\Sigma} \tau}{2}+\left(\gamma_{\uparrow}-\gamma_{\downarrow}\right)\left(\mathcal{N}-\frac{1}{2}\right) \tau} \theta[\mathcal{N}(1-\mathcal{N})] \\
P_{2}(\tau, \mathcal{N})=\frac{\gamma_{\uparrow} \gamma_{\downarrow} \tau^{2}}{\gamma_{\Sigma}} e^{-\frac{\gamma_{\Sigma}}{2} \tau+\left(\gamma_{\uparrow}-\gamma_{\downarrow}\right)\left(\mathcal{N}-\frac{1}{2}\right) \tau}\left[\gamma_{\uparrow} \mathcal{N}+\gamma_{\downarrow}(1-\mathcal{N})\right] \theta[\mathcal{N}(1-\mathcal{N})] \\
P_{3}(\tau, \mathcal{N})=2 \frac{\left(\gamma_{\uparrow} \gamma_{\downarrow}\right)^{2} \tau^{3}}{\gamma_{\Sigma}} e^{-\frac{\gamma_{\Sigma} \tau}{2}+\left(\gamma_{\uparrow}-\gamma_{\downarrow}\right)\left(\mathcal{N}-\frac{1}{2}\right) \tau} \mathcal{N}(1-\mathcal{N}) \theta[\mathcal{N}(1-\mathcal{N})] \\
\text { and } \quad P_{4}(\tau, \mathcal{N})=\frac{\left(\gamma_{\uparrow} \gamma_{\downarrow} \tau^{2}\right)^{2}}{2 \gamma_{\Sigma}} e^{-\frac{\gamma_{\Sigma}}{2} \tau+\left(\gamma_{\uparrow}-\gamma_{\downarrow}\right)\left(\mathcal{N}-\frac{1}{2}\right) \tau} \\
\quad \times \mathcal{N}(1-\mathcal{N})\left[\gamma_{\uparrow} \mathcal{N}+\gamma_{\downarrow}(1-\mathcal{N})\right] \theta[\mathcal{N}(1-\mathcal{N})]
\end{gathered}
$$

The distributions $P_{m}(\tau, \mathcal{N})$ for the first four jumps are plotted in Fig. 4.3 (a). With an increase in number of jumps, the peak of distribution becomes prominent at the mean value, i.e., $\langle\mathcal{N}\rangle=\gamma_{\uparrow} / \gamma_{\Sigma}$.

Figure 4.3 (b) shows the change in total distribution $\sum_{m=0}^{k} P_{m}(\tau, \mathcal{N})$ with an increase in the number of jumps $k$. This shows a rising trend with an increase in the number of jumps $k$ considered for the calculation of the sum $\sum_{m=0}^{k} P_{m}(\tau, \mathcal{N})$. This is also observed in the experimental time averaged current distribution (Fig. 4.4) with increase in averaging-time $\tau$ for small $\tau$, e.g., change from $2 \mathrm{~ms}$ to $4 \mathrm{~ms}$.

The results presented in Fig. 4.3 are for a system with total transition rate $\gamma_{\Sigma}=400 \mathrm{~Hz}$ and averaging-time $\tau=10 \mathrm{~ms}$. This gives, the average number of jumps system goes through in the time $\tau$ as $2 \gamma_{\uparrow} \gamma_{\downarrow} \tau / \gamma_{\Sigma}=1.5$. Thus, the number of jumps larger than $k=4$ has only a tiny effect on the total distribution. As seen from Fig. 4.3, the distribution is limited between 0 and 1 as expected because the system jumps between states with 0 and 1 extra electrons on the island.

The distributions $P_{m}(\tau, \mathcal{N})$ and $\sum_{m=0}^{k} P_{m}(\tau, \mathcal{N})$ presented in Fig. 4.3 correspond to the time-averaged number of extra electrons on the island. The measured distribution of time-averaged normalized current $\mathcal{P}(\mathcal{I}, \tau)(4.16)$ is presented in Fig. 4.4. The Gaussian shape of the distribution in small averaging-time (turquoise) is due to an addition of the noise term to the distribution. This is due to the fact that the delta function in Eqs. (4.22) and (4.25) is replaced by the distribution of noise in the detector output current $I_{d}$ after the convolution (4.26). This noise being Gaussian distributed gives the shape to time-averaged current distributions $\mathcal{P}(\mathcal{I}, \tau)$.

An increase in $\tau$ reduces the presence of noise in the time-averaged current, as the larger $\tau$ ensures the averaging of noise component of the signal over larger time. This results in the narrowing of the Gaussian peak with increase in averaging-time, e.g., $\tau=80 \mathrm{~ms}$ and $320 \mathrm{~ms}$ in Fig. 4.4. Consequently, the distribution $\mathcal{P}(\mathcal{I}, \tau)$ is dominated by the jumps between system states, and the noise term $\tilde{\xi}_{\tau}$ can be ignored for long averaging times. Next, we discuss the properties of $\mathcal{P}(\mathcal{I}, \tau)$ for large $\tau$. 

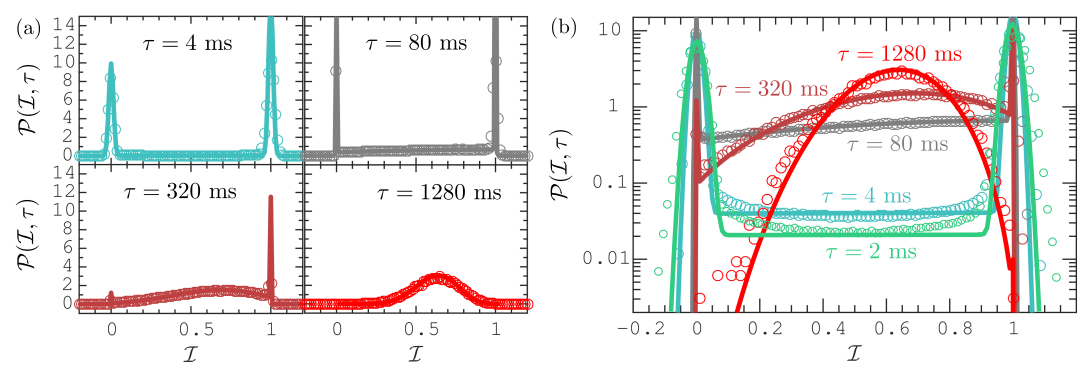

Figure 4.4. The variation of probability distribution of time-averaged normalized current $\mathcal{I}$ with averaging-time $\tau$ on (a) linear and (b) log scale. Different colors correspond to different $\tau$ s mentioned in the legend. The theory curves (solid lines) based on Eqs. (4.25) and (4.26) are in agreement with the experiments (open symbols), without any extra fit parameter. The transition rates are $\gamma_{\uparrow}=130 \mathrm{~Hz}$ and $\gamma_{\downarrow}=70 \mathrm{~Hz}$. Adapted from Publication II.

\subsubsection{Long-time limit}

For averaging-time $\tau \gg 1 / \gamma_{\Sigma}$, the first two terms in distribution (4.25) are insignificant and the modified Bessel function can be approximated as $I_{0}(x) \simeq I_{1}(x) \simeq e^{x} / \sqrt{2 \pi x}$. In this approximation, the distribution eventually takes on the large-deviation form [5] $\mathcal{P}(\mathcal{I}, \tau) \propto e^{-\theta(\mathcal{I}) \tau}$ with the convex rate function $\theta\left((\mathcal{I})=\left(\sqrt{\gamma_{\uparrow}(1-\mathcal{I})}-\sqrt{\gamma_{\downarrow} \mathcal{I}}\right)^{2}\right.$ following directly from Eq. (4.25). The resulting distribution

$$
\frac{\ln \mathcal{P}(\mathcal{I}, \tau)}{\tau} \simeq-\frac{\left[\sqrt{\gamma_{\uparrow}\left(\mathcal{I}_{1}-\mathcal{I}\right)}-\sqrt{\gamma_{\downarrow}\left(\mathcal{I}-\mathcal{I}_{0}\right)}\right]^{2}}{\mathcal{I}_{1}-\mathcal{I}_{0}}
$$

is in agreement with the previously found expression using the saddle point approximation [106] and explains the elliptic behavior of distribution $\mathcal{P}(\mathcal{I}, \tau)$ in sufficiently long-time limit (see Fig. 4.4 and Refs. [87, 116, 118, 119]). The terms $\mathcal{I}_{1}=1$ and $\mathcal{I}_{0}=0$ are the average normalized currents corresponding to system state 1 and 0 , respectively.

The transition rates between the two states of the system along with the currents $\mathcal{I}_{1}$ and $\mathcal{I}_{0}$ define the width and the center of probability distribution $\mathcal{P}(\mathcal{I}, \tau)$. The distribution is peaked at the mean current given by $\langle\mathcal{I}\rangle=\gamma_{\uparrow} / \gamma_{\Sigma}$, while the spread is given by the product of transition rates.

To observe this dependence in experiments, we plot the logarithm of the probability distribution $\mathcal{P}(\mathcal{I}, \tau)$ rescaled by averaging-time $\tau=400 \mathrm{~ms}$ for different transition rates (see caption) in Fig. 4.5 (a). The distribution obtained from experiments (symbols) are in agreement with the theory (4.31) (solid lines) using the transition rates $\gamma_{\uparrow}$ and $\gamma_{\downarrow}$ computed from the measured data.

Using the distribution (4.31), we define

$$
\mathcal{G}(\mathcal{I}) \equiv \frac{1}{2 \sqrt{\gamma_{\uparrow} \gamma_{\downarrow}}}\left\{\frac{\ln \mathcal{P}(\mathcal{I}, \tau)}{\tau}+\frac{\gamma_{\uparrow} \Delta \mathcal{I}_{1}-\gamma_{\downarrow} \Delta \mathcal{I}_{0}}{\mathcal{I}_{1}-\mathcal{I}_{0}}\right\}
$$



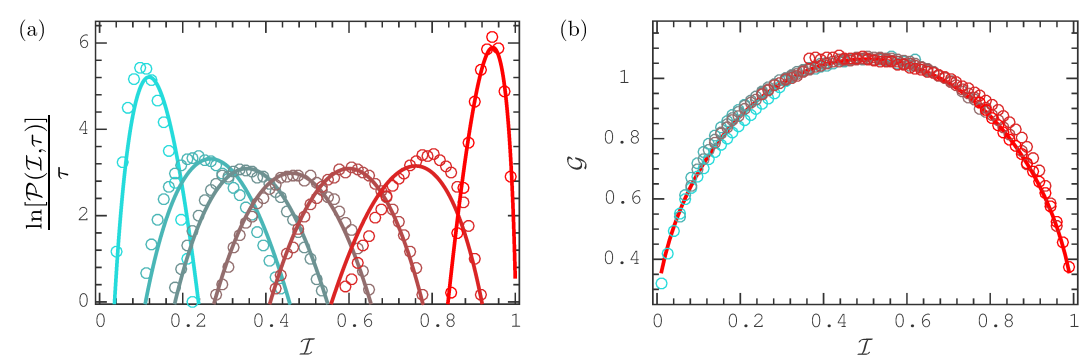

Figure 4.5. (a) The ratio of logarithm probability distribution to the averaging-time, $\ln \mathcal{P} / \tau$, for large averaging-time $\tau=400 \mathrm{~ms}$. The colors represent curves corresponding to different transition rates (in $\mathrm{Hz}$ ), $\gamma_{\uparrow} / \gamma_{\downarrow}=40 / 315$ (turquoise), $109 / 248,85 / 187,88 / 98,120 / 81,166 / 55$, and 285/20 (red). The symbols are experimental data and solid lines are the theory plots based on Eq. (4.31) and rates computed from experimental data. (b) The universal semicircle obtained after transforming the experimental data (symbols) using Eq. (4.32) and the red solid line is the universal semicircle (4.33). Adapted from Publication II.

This function does not depend on the system parameters, i.e., the transition rates between the two states, $\gamma_{\uparrow}$ and $\gamma_{\downarrow}$. Here, we have defined the terms $\Delta \mathcal{I}_{0}=\mathcal{I}-\mathcal{I}_{0}$ and $\Delta \mathcal{I}_{1}=\mathcal{I}-\mathcal{I}_{1}$ as the departure of time-averaged normalized current $\mathcal{I}$ from the mean currents $\mathcal{I}_{0}$ and $\mathcal{I}_{1}$ corresponding to system states 0 and 1 , respectively. The second term absorbs the tilt of the elliptic distribution (4.31) and Eq. (4.32) can be re-written as

$$
[\mathcal{G}(\mathcal{I})]^{2}+\left[\frac{\mathcal{I}-\mathcal{I}_{\text {mean }}}{\mathcal{I}_{1}-\mathcal{I}_{0}}\right]^{2}=1 .
$$

Here, the mean current equals $\mathcal{I}_{\text {mean }}=\left(\mathcal{I}_{0}+\mathcal{I}_{1}\right) / 2$. The expression (4.33) results in an universal semicircle (see Fig. 4.5 (b) and Publication II for details) and is valid for any bistable system irrespective of its microscopic details. In Fig. $4.5(\mathrm{~b})$, we present $\mathcal{G}(\mathcal{I})$ using the experimental data (symbols) for different transition rates as in panel (a). The plot for different rates collapse to an universal semicircle as expected from Eq. (4.33).

A further investigation of the distribution $\mathcal{P}(\mathcal{I}, \tau)$ in long-time limit (see Eq. (4.31)) leads us to a fluctuation relation

$$
\frac{1}{\tau} \ln \left[\frac{\mathcal{P}\left(\mathcal{I}=\mathcal{I}_{\text {mean }}+\mathcal{J}, \tau\right)}{\mathcal{P}\left(\mathcal{I}=\mathcal{I}_{\text {mean }}-\mathcal{J}, \tau\right)}\right]=\Omega \mathcal{J}
$$

We observe the departure of average current $\mathcal{J}$ from its mean value $\mathcal{I}_{\text {mean }}=$ 0.5 for the normalized time-averaged current $\mathcal{I}$. The slope $\Omega=2\left(\gamma_{\uparrow}-\right.$ $\left.\gamma_{\downarrow}\right) /\left(\mathcal{I}_{1}-\mathcal{I}_{0}\right)$ is a system dependent parameter and should hold for any bistable system. In Fig. 4.6, we plot the left hand side of Eq. (4.34) for data (symbols) from single-island experiment (same data set as presented in Fig. 4.5) and the theory (4.34) with rates computed from experimental data. We find a good agreement between the experimental results and the expected theory. The relation (4.34) is similar to that of GallavottiCohen fluctuation theorem $[21,120]$ apart from the physical entity under 


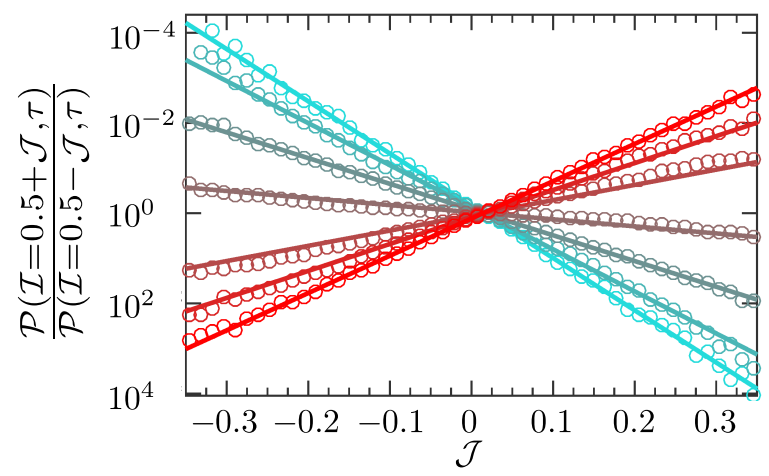

Figure 4.6. Fluctuation relation (4.34) for the probability distribution of time-averaged current $\mathcal{P}(\mathcal{I}, \tau)$. The symbols are experimental data from the single-island sample (Fig. 2.3), and solid lines are theory, right-hand side of Eq. (4.34), with transition rates obtained from experiments. Adapted from Publication II.

investigation. Here, we study the departure of the average current $\mathcal{J}$ from $\mathcal{I}_{\text {mean }}$ instead of a thermodynamic quantity, intensive entropy production, studied in Gallavotti-Cohen fluctuation theorem. 
Current distribution of a bistable system 


\section{Stochastic thermodynamics of an electronic double dot}

As mentioned in the introduction, the usual thermodynamics of averages is not enough to describe the fluctuations in physical properties of small systems. The field of stochastic thermodynamics overcomes this limitation by generalizing these concepts to systems where the fluctuations are prominent. This is done by treating the thermodynamic quantities at the trajectory level.

In this chapter, we discuss the stochastic entropy production and the extreme values it can attain. The double dot system, described in Sec. 2.4, offers an excellent test bench for these extreme-value statistics by providing a tool to determine the direction of electron transitions through the device. This chapter presents the experimental results of Publication III and theoretical concepts needed for it.

We start with a brief note on a Markovian master equation (already mentioned in Eq. (2.20)) and its time reversed counterpart needed for the definition of stochastic entropy production. Next, we define the stochastic entropy production and present the fluctuation relations. Last, we present the distribution for the extreme values of the entropy produced in a given time for the double dot system (Sec. 2.4).

\subsection{Master equation}

We focus on Markovian dynamics of a system governed by the master equation (2.20), namely,

$$
\frac{d p(n, t)}{d t}=\sum_{m(\neq n)} \Gamma_{m \rightarrow n}(t) p(m, t)-\left(\sum_{m(\neq n)} \Gamma_{n \rightarrow m}(t)\right) p(n, t) .
$$

Here, $\Gamma_{m \rightarrow n}(t)$ is the transition rate from the initial state $m$ to the final state $n$ and $p(n, t)$ is the occupation probability of the state $n$ at time $t$. For simplicity, we assume that for every possible jump $m \rightarrow n$, there exists a possibility for jump in the reverse direction, i.e., $\Gamma_{n \rightarrow m} \neq 0$. 
The dynamics of backward (time-reversed) process is given by [36, 96]

$$
\frac{d \tilde{p}(n, \tilde{t})}{d \tilde{t}}=\sum_{m(\neq n)} \Gamma_{m \rightarrow n}(\tau-\tilde{t}) \tilde{p}(m, \tilde{t})-\left(\sum_{m(\neq n)} \Gamma_{n \rightarrow m}(\tau-\tilde{t})\right) \tilde{p}(n, \tilde{t}),
$$

where the time corresponding to the reversed process is $\tilde{t}=\tau-t$ with a reference time $\tau$. Next, we define the stochastic entropy production for such Markovian processes.

\subsection{Stochastic entropy production}

We define $P\left(\left\{n_{i}, t_{i}\right\}_{i=0}^{N(\tau)}\right)$ as the probability of having a stochastic trajectory such that the state of the system is $n_{0}$ at initial time $t_{0}=0$, the state changes from $n_{i-1}$ to $n_{i}$ at time $t_{i}$ with no change in state from time $t_{i-1}$ to $t_{i}$, and the trajectory ends at time $\tau$ with no jump in the state from time $t_{N}$ to $\tau . N(\tau)$ is the total number of changes in the system state during time $\tau$. The stochastic entropy production [17, 37] associated with such trajectory is given by

$$
\Delta S(\tau)=k_{\mathrm{B}} \ln \frac{P\left(\left\{n_{i}, t_{i}\right\}_{i=0}^{N(\tau)}\right)}{\tilde{P}\left(\left\{n_{i}, \tilde{t}_{i+1}\right\}_{i=N(\tau)}^{0}\right)} .
$$

Here, $\tilde{P}\left(\left\{n_{i}, \tilde{t}_{i+1}\right\}_{i=N(\tau)}^{0}\right)$ denotes the probability to obtain the corresponding reversed trajectory in the time-reversed process with $t_{N+1}$ denoting $\tau$.

An example of a stochastic trajectory obtained from the double dot experiment is shown in Fig. 5.1 (a). The jumps in the trajectory correspond to the change in charge state of the system. Figure 5.1 (b) shows the stochastic entropy production for the trajectory in panel (a). We discuss below how to obtain the time trace of stochastic entropy production using Eq. (5.2) and the time evolution of the system state.

Using the Markovian master equations (2.20) and (5.1), for which the probability of a jump $n_{i} \rightarrow n_{i+1}$ is given by $\Gamma_{n_{i} \rightarrow n_{i+1}} \delta t$ and the probability of no jump is given by $\exp \left(-\delta t \sum_{y} \Gamma_{n_{i} \rightarrow y}\right)$ with a short time step $\delta t[36,96]$, the entropy production (5.2) associated with the trajectory simplifies to

$$
\frac{\Delta S(\tau)}{k_{\mathrm{B}}}=\ln \frac{p\left(n_{0}, 0\right)}{\tilde{p}\left(n_{N}, 0\right)}+\sum_{i=1}^{N(\tau)} \ln \frac{\Gamma_{n_{i-1} \rightarrow n_{i}}\left(t_{i}\right)}{\Gamma_{n_{i} \rightarrow n_{i-1}}\left(t_{i}\right)} .
$$

Here, $p\left(n_{0}, 0\right)$ is the probability of the system to be in state $n_{0}$ at time $t=0$ and $\tilde{p}\left(n_{N}, 0\right)$ is the probability of the system to be in state $n_{N}$ at the beginning of backward trajectory, i.e., $\tilde{t}=0$. Considering the process to be stationary, the transition rates in Eqs. (2.20) and (5.1) are time independent, and hence the master equations are equivalent. In this case, we use the steady state solution of the master equations as the 

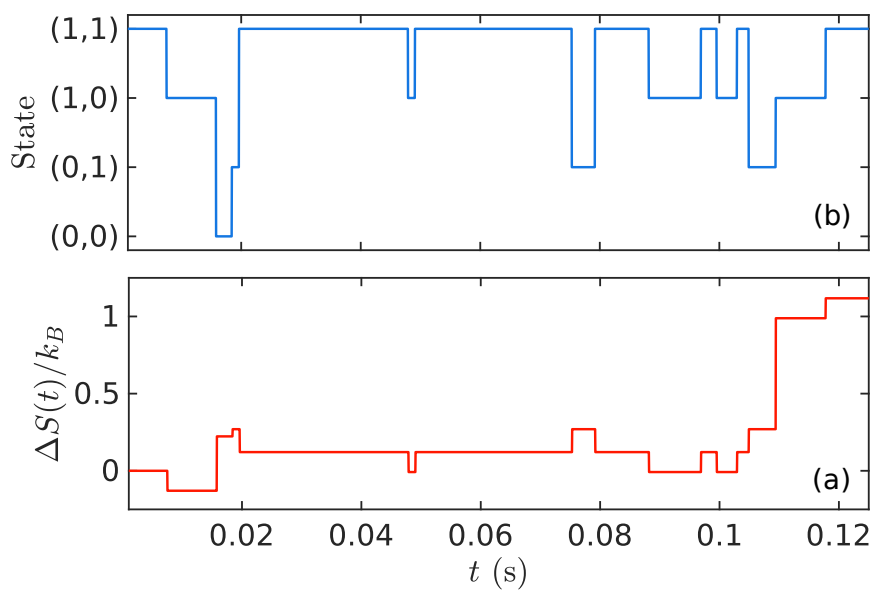

Figure 5.1. (a) A sample experimental trajectory of change in double dot charge state at bias voltage $V_{\mathrm{b}}=90 \mu \mathrm{V}$. (b) The corresponding stochastic entropy production computed using Eq. (5.8).

initial probabilities of forward and backward processes. Consequently, the entropy production (5.3) for the trajectory with stationary Markovian dynamics becomes $[24,121]$

$$
\frac{\Delta S(\tau)}{k_{\mathrm{B}}}=\ln \frac{p\left(n_{0}\right)}{p\left(n_{N}\right)}+\sum_{i=1}^{N(\tau)} \ln \frac{\Gamma_{n_{i-1} \rightarrow n_{i}}}{\Gamma_{n_{i} \rightarrow n_{i-1}}} .
$$

Here, we have dropped the time argument from the probabilities of system states and the transition rates because they are time-independent for a stationary process. The Eq. (5.4) can be re-written as

$$
\frac{\Delta S(\tau)}{k_{\mathrm{B}}}=\sum_{i=0}^{N(\tau)}\left[\ln \frac{p\left(n_{i-1}\right)}{p\left(n_{i}\right)}+\ln \frac{\Gamma_{n_{i-1} \rightarrow n_{i}}}{\Gamma_{n_{i} \rightarrow n_{i-1}}}\right]=\sum_{i=0}^{N(\tau)} \frac{\Delta S_{n_{i} \rightarrow n_{i+1}}}{k_{\mathrm{B}}},
$$

where the entropy production associated with the transition of the state of the system from $n_{i-1}$ to $n_{i}\left(\Delta S_{n_{i-1} \rightarrow n_{i}}\right)$ is the sum of the change in system entropy $^{1}$

$$
\Delta S_{n_{i-1} \rightarrow n_{i}}^{\mathrm{sys}}=k_{\mathrm{B}} \ln \left[\frac{p\left(n_{i-1}\right)}{p\left(n_{i}\right)}\right]
$$

and the entropy flow to the environment

$$
\Delta S_{n_{i-1} \rightarrow n_{i}}^{\mathrm{env}}=k_{\mathrm{B}} \ln \left[\frac{\Gamma_{n_{i-1} \rightarrow n_{i}}}{\Gamma_{n_{i} \rightarrow n_{i-1}}}\right]
$$

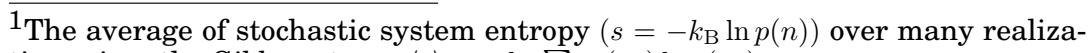
tions gives the Gibbs entropy $\langle s\rangle=-k_{\mathrm{B}} \sum_{j} p\left(n_{j}\right) \ln p\left(n_{j}\right)$.
} 

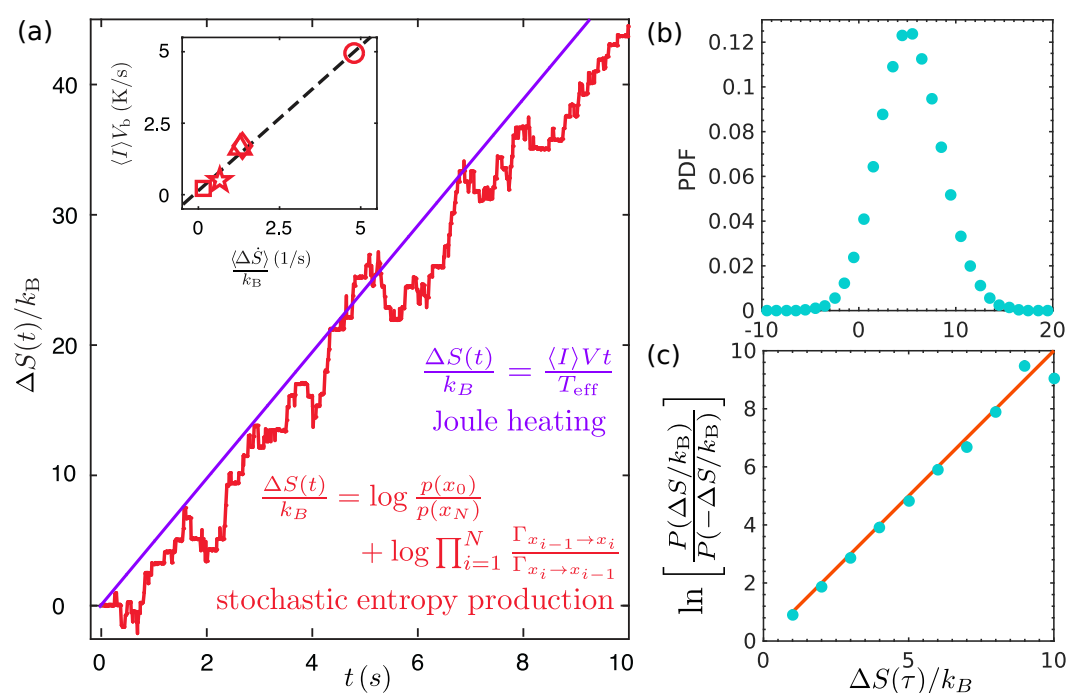

Figure 5.2. (a) The experimental time dependence of stochastic entropy production (5.8) (red) and average Joule heating (purple) in the double dot with bias voltage $V_{\mathrm{b}}=90 \mu \mathrm{V}$. Inset: the linear dependence of average Joule dissipation rate $\langle I\rangle V_{\mathrm{b}}$ on the average entropy production rate $\langle\dot{S}\rangle$ for different bias voltages, with slope given by $T_{\text {eff }}=(1.01 \pm 0.16) \mathrm{K}$, see Eq. (2.32). (b) The probability distribution function (PDF) of the entropy production for the double dot at $V_{\mathrm{b}}=90 \mu \mathrm{V}$ computed with $\sim 3 \times 10^{5}$ realizations of entropy production in time $\tau=1 \mathrm{~s}$. (c) The experimental test of Eq. (5.13) (line) for data (symbols) in (b).

\subsubsection{Stochastic entropy production in an electronic double dot}

The total entropy production (5.5) can be re-written as

$$
\frac{\Delta S(t)}{k_{\mathrm{B}}}=\sum_{i=1}^{N(\tau)} \ln \frac{\mathcal{N}_{n_{j-1} \rightarrow n_{j}}}{\mathcal{N}_{n_{j} \rightarrow n_{j-1}}},
$$

where $\mathcal{N}_{n \rightarrow m}$ is the number of jumps from state $n$ to $m$ integrated over all realizations to approximate the steady state tunneling rates in Eq. (2.29) (namely, $\Gamma_{n \rightarrow m}^{\exp }=\mathcal{N}_{n \rightarrow m} /\{p(n) \tau\}$ ), while $N(\tau)$ is the total number of jumps in a single experimental trace. In the experiments with double dot sample, we use Eq. (5.8) to compute the stochastic entropy production corresponding to the system state trace. For example, Fig. 5.1 (b) and Fig. 2.7 (c) show the stochastic entropy production for the time evolution of system state shown in Fig. 5.1 (a) and Fig. 2.7 (b), respectively. Figure 5.2 (a) shows a similar time trace of sample stochastic entropy production for the double dot system (red line) when kept at bias $V_{\mathrm{b}}=90 \mu \mathrm{V}$.

At equilibrium, the total number of jumps between any two states are the same in the forward and reverse directions, resulting in the entropy production $\Delta S(t)=0$ at all times $t$. In experiments, this could be attained by setting the device bias voltage to 0 . When driven out of equilibrium, the total entropy of the system, here double dot + environment (which includes 
detectors, substrate), increases monotonically in time on average.

In a nonequilibrium steady state, both $\Delta S(t)$ and entropy flow to the environment $\Delta S^{\text {env }}(t)$ increase on average with time, i.e., $\langle\Delta S(t)\rangle>0$ and $\left\langle\Delta S^{\mathrm{env}}(t)\right\rangle>0$, in accord with the second law of thermodynamics. This is realized in the double dot system (Fig. 2.4) by a DC bias voltage $V_{\mathrm{b}}$ applied to one of its lead keeping the other lead grounded and the gates fixed. If the environment consists of multiple thermal reservoirs with temperatures $T_{k}$ and the local detailed balance holds, then the entropy flow to the environment can be written as $\Delta S^{\text {env }} / k_{\mathrm{B}}=-\sum_{k} Q_{k}(t) / T_{k}$ with $Q_{k}$ being the heat production in the $k$ th reservoir.

If all the reservoirs are at same temperature $T, \Delta S^{\text {env }}$ reduces to the usual thermodynamic definition of entropy,

$$
\Delta S^{\mathrm{env}}(t)=\frac{-Q(t)}{T},
$$

where the total heat production is defined as $Q(t)=\sum_{k} Q_{k}(t)$. In this case, the system obeys detailed balance (2.31) with a fixed temperature $T$.

In the double dot experiments, due to the transition rates being an average over the charge states of detector, the detailed balance (2.31) does not hold for the system. However, we can use Eq. (2.32) to define an effective temperature $T_{\text {eff }}$ as a proportionality constant between the Joule dissipated power (given by $\langle\dot{Q}(t)\rangle=\langle I\rangle V_{\mathrm{b}}$ with $\langle I\rangle$ as the average current through the system at bias voltage $V_{\mathrm{b}}$ ) and the average rate of stochastic entropy production $\langle\Delta \dot{S}\rangle$, see the inset of Fig. 5.2 (a). Using this $T_{\text {eff }}$, Eqs. (5.7) and (5.9), we can estimate the heat exchange between the system and environment. We present the distribution of average minimum entropy flow to the environment and its relation to the average of maximum heat exchange with the environment in Sec. 5.4.

Figure 5.2 (a) shows the Joule heating (purple line) in the system due to a net flow of electrons from one lead to another. This captures the average increase in entropy and we infer that the total stochastic entropy production $\Delta S(t)$ in the system is dominated by the heat dissipated because of a net current through the system. In Fig. 5.2 (b) the probability distribution of stochastic entropy production $\Delta S(\tau)$ at a fixed time $\tau=1 \mathrm{~s}$ in the double dot with $V_{\mathrm{b}}=90 \mu \mathrm{V}$ is presented. In the following section, we discuss briefly the properties of such distribution.

\subsection{Distribution of entropy}

In this section, we first discuss the properties of entropy production defined by Eq. (5.2) along with the fluctuation relations. In the end, an experimental test of detailed fluctuation relation is presented for the stochastic entropy production in an electronic double dot.

Taking average of $e^{-\Delta S(\tau) / k_{\mathrm{B}}}$ over the possible forward trajectories, Eq. (5.2) 
gives the following expression [122]

$$
\begin{aligned}
\left\langle e^{-\Delta S(\tau) / k_{\mathrm{B}}}\right\rangle & =\sum_{\substack{\text { forward } \\
\text { trajectories }}} \frac{\tilde{P}\left(\left\{n_{i}, \tilde{t}_{i+1}\right\}_{i=N(\tau)}^{0}\right)}{P\left(\left\{n_{i}, t_{i}\right\}_{i=0}^{N(\tau)}\right)} P\left(\left\{n_{i}, t_{i}\right\}_{i=0}^{N(\tau)}\right) \\
& =\sum_{\substack{\text { forward } \\
\text { trajectories }}} \tilde{P}\left(\left\{n_{i}, \tilde{t}_{i+1}\right\}_{i=N(\tau)}^{0}\right) \leq 1
\end{aligned}
$$

Here, using Jensen inequality, we obtain $\langle\Delta S(\tau)\rangle \geq 0$ which is in accord with the second law of thermodynamics. This result is true even without the assumption done in Eqs. (2.20) and (5.1) for the transition rates, and is a direct consequence of Gibbs' inequality [123]. The equality

$$
\left\langle e^{-\Delta S(\tau) / k_{\mathrm{B}}}\right\rangle=1
$$

is obtained by assuming that all the initial states of the backward trajectories have a non-zero probability to be an end state of the forward process and all the end states of the reverse process have a non-zero probability to be an initial state of the forward process. Together with the assumption for transition rates in master equation (2.20), this gives that if $P\left(\left\{n_{i}, t_{i}\right\}_{i=0}^{N(\tau)}\right)=0$, then $\tilde{P}\left(\left\{n_{i}, \tilde{t}_{i+1}\right\}_{i=N(\tau)}^{0}\right)=0$, i.e., the backward probability is absolutely continuous [123] with respect to the forward probability. This relation (5.11) is known as the integral fluctuation relation [16, 121, 124, 125]. It breaks down [126], i.e., $\left\langle e^{-\Delta S(\tau) / k_{\mathrm{B}}}\right\rangle<1$, for the absolute irreversible case [127]. In the case of a stationary process, the expression (5.11) is valid as we assume that the initial distributions follow the steady state solution.

The probability to obtain entropy $\Delta S(\tau) / k_{\mathrm{B}}=s$ for a forward process is given by [122]

$$
\begin{aligned}
P\left(\Delta S / k_{\mathrm{B}}=s, \tau\right) & =\sum_{\substack{\text { forward } \\
\text { trajectories }}} P\left(\left\{n_{i}, t_{i}\right\}_{i=0}^{N(\tau)}\right) \delta_{P\left(\left\{n_{i}, t_{i}\right\}_{i=0}^{N(\tau)}\right), \exp (s) \tilde{P}\left(\left\{n_{i}, \tilde{t}_{i+1}\right\}_{i=N(\tau)}^{0}\right)} \\
& =e^{s} \sum_{\substack{\text { forward } \\
\text { trajectories }}} \tilde{P}\left(\left\{n_{i}, \tilde{t}_{i+1}\right\}_{i=N(\tau)}^{0}\right) \delta_{\tilde{P}\left(\left\{n_{i}, \tilde{t}_{i+1}\right\}_{i=N(\tau)}^{0}\right), \exp (-s) P\left(\left\{n_{i}, t_{i}\right\}_{i=0}^{N(\tau)}\right)} \\
& =e^{s} \tilde{P}\left(\Delta S / k_{\mathrm{B}}=-s, \tau\right), \quad \text { for } \quad s \in \mathbb{R} \backslash\{-\infty, \infty\},
\end{aligned}
$$

where the last equality follows by defining that the reverse process of the reserve process is the original forward process. This is known as the detailed fluctuation relation [17, 37, 125, 128]. In the case of steady state, it simplifies to

$$
\frac{P\left(\Delta S / k_{\mathrm{B}}=s, \tau\right)}{P\left(\Delta S / k_{\mathrm{B}}=-s, \tau\right)}=e^{s},
$$

shown experimentally in Fig. 5.2 (c). Here, $\tilde{P}\left(\Delta S / k_{\mathrm{B}}=-s, \tau\right)=P\left(\Delta S / k_{\mathrm{B}}=\right.$ $-s, \tau)$ because the transition rates in the stationary process are time independent and we have chosen the initial distributions of both forward 
and backward process to be the same. As a consequence of Eq. (5.13), the cumulative distribution of entropy can be written as

$$
P\left(\Delta S / k_{\mathrm{B}} \geq-s, \tau\right) \geq 1-e^{-s}, \quad s \geq 0 .
$$

This provides the bound for entropy produced at a given time $\tau$ to have a value larger than or equal to a negative entropy value, here $-s$. An experimental test of detailed fluctuation relation (5.13) is presented in Fig. 5.2 (c). The experimental result (symbols), obtained from the distribution in (b), is in agreement with the expected theory (5.13) line.

\subsection{Extreme values of entropy}

Another way of quantifying the occurrence of negative entropy production is to look at the minimum amount of entropy produced within the time $t$. For this, we define $\Delta S_{\min }(t)=\min _{0 \leq t^{\prime} \leq t} \Delta S\left(t^{\prime}\right)$ as the minimum entropy produced in time $t$.

In this section, we present the statistics of $\Delta S_{\min }(t)$, theoretically studied in Ref. [38] and the results of experiments on double dot presented in Publication III. We start with a sketch of the proof of Eqs. (5.17) and (5.18), originally done in Ref. [38]. We then present the experimental test of Eqs. (5.17) and (5.18) and provide a bound on the maximum heat exchange between the environment and double dot system (5.20).

For a stationary process, the entropy production is given by Eq. (5.5), and the exponentiated entropy $e^{-\Delta S(t) / k_{\mathrm{B}}}$ is always a non-negative quantity and has been shown to be martingale [129-133] in time (see Ref. [38] for details on derivation), i.e.,

$$
\left\langle e^{-\Delta S(t) / k_{\mathrm{B}}} \mid\left\{s_{i}, t_{i}\right\}_{i=0}^{n}\right\rangle=e^{-s_{n} / k_{\mathrm{B}}}, \quad t \geq t_{n} .
$$

Here we use the notation $\langle X \mid Y\rangle \equiv \int X P(X \mid Y) d X$ for the conditional expectation of $\mathrm{X}$ given $\mathrm{Y}, P(X \mid Y)$ for the conditional probability density of $\mathrm{X}$ given $\mathrm{Y}$ and $\left\{s_{i}, t_{i}\right\}_{0}^{n}$ for a specific trajectory of stochastic entropy production which attains value $s_{i}$ at time $t_{i}$ from time $t_{0}=0$ to $t_{n}$. A trivial implication of Eq. (5.15) is the integral fluctuation theorem, which is recovered in the case we specify the trajectory $\left\{s_{i}, t_{i}\right\}_{i=0}^{n=0}$ as at $t_{0}=0, s_{0}=0$.

The martingality of $e^{-\Delta S(t) / k_{\mathrm{B}}}$ allows us to use the properties of martingale, such as Doob's inequality [130]:

$$
P\left(\max _{0 \leq t^{\prime} \leq t} e^{-\Delta S\left(t^{\prime}\right) / k_{\mathrm{B}}} \geq C\right) \leq \frac{\left\langle e^{-\Delta S(t) / k_{\mathrm{B}}}\right\rangle}{C}=\frac{1}{C}, \quad \forall C>0 .
$$

Here, we have used Eq. (5.11) for the right hand side of the expression. Equation (5.16) can be equivalently written as

$$
P\left(\min _{0 \leq t^{\prime} \leq t} \Delta S\left(t^{\prime}\right) / k_{\mathrm{B}} \leq-s\right) \leq e^{-s}, \quad s \geq 0
$$



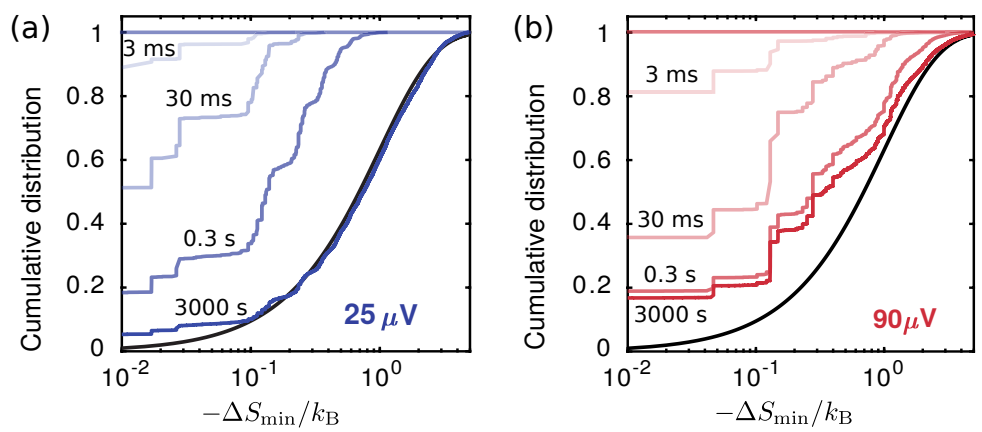

Figure 5.3. The cumulative distribution (5.17) of minimum entropy production in time $t, \Delta S_{\min }=\min _{0 \leq x \leq t} \Delta S(x)$, for the double dot with bias (a) $V_{\mathrm{b}}=25 \mu \mathrm{V}$ and (b) $V_{\mathrm{b}}=90 \mu \mathrm{V}$ for different values of time (see legend). The black lines are theory curves $1-e^{\Delta S_{\min } / k_{\mathrm{B}}}(5.17)$ [38]. Adapted from Publication III.

where $s=\ln C$ and we have restricted $s \geq 0$ because naturally probability is upper bounded by 1 . The bound (5.17) can be re-written as $P\left(\Delta S_{\min }(t) \geq-s\right) \geq 1-e^{-s}$, see Fig. 5.3. In Fig. 5.3, we plot the cumulative distribution $P\left(\Delta S_{\min }(t) \geq-s\right)$ for the double dot sample with bias voltage $V_{\mathrm{b}}$. The experimental data (colored lines) obey the bound $1-e^{-s}$ (black lines).

Integration of (5.17) over all the possible values of $s$, gives the bound for the minimum entropy [38]:

$$
\left\langle\Delta S_{\min }(t)\right\rangle \geq-k_{\mathrm{B}}
$$

see Fig. 5.4 (a). Figure 5.4 (a) demonstrates the validity of the bound (5.18) in the double dot experiment for bias $V_{\mathrm{b}}$ varying from $-50 \mu \mathrm{V}$ to $90 \mu \mathrm{V}$. The bound is tightest for $V_{\mathrm{b}}=25 \mu \mathrm{V}$, when the jumps in entropy production $\Delta S_{n_{i-1} \rightarrow n_{i}}$ [defined in Eq. (5.5)] are $\ll k_{\mathrm{B}}$ and the time trace of entropy production (5.4) can therefore be approximated as a continuous process for which $\left\langle\Delta S_{\min }(t)\right\rangle=-k_{\mathrm{B}}[38,134]$. Using the definition of entropy exchange with environment $\Delta S^{\text {env }}(5.7)$, we obtain a bound for minimum $\Delta S^{\text {env }}$ as

$$
\left\langle\Delta S_{\min }^{\mathrm{env}}(t)\right\rangle \geq-k_{\mathrm{B}}\left(1+\sum_{n} p(n) \ln \frac{p(n)}{p_{\min }}\right),
$$

see Fig. 5.4 (b). Here, $p_{\min }=\min _{n} p(n)$ is the stationary probability corresponding to the least probable state. In Fig. 5.4 (b), we present the experimental observation of $\left\langle\Delta S_{\min }^{\mathrm{env}}(t)\right\rangle$ for different bias voltages across the double dot system. The experiments (symbols) are in agreement with the bound of Eq. (5.19) (dashed lines). For a system with all the states equiprobable, the second term in the right hand side of Eq. (5.19) is 0. This recovers Eq. (5.18) as $\Delta S_{m \rightarrow n}^{\text {sys }}$ is always zero.

For a system in thermal equilibrium at temperature $T$, the heat and entropy exchange with environment are related by Eq. (5.9). Consequently, 

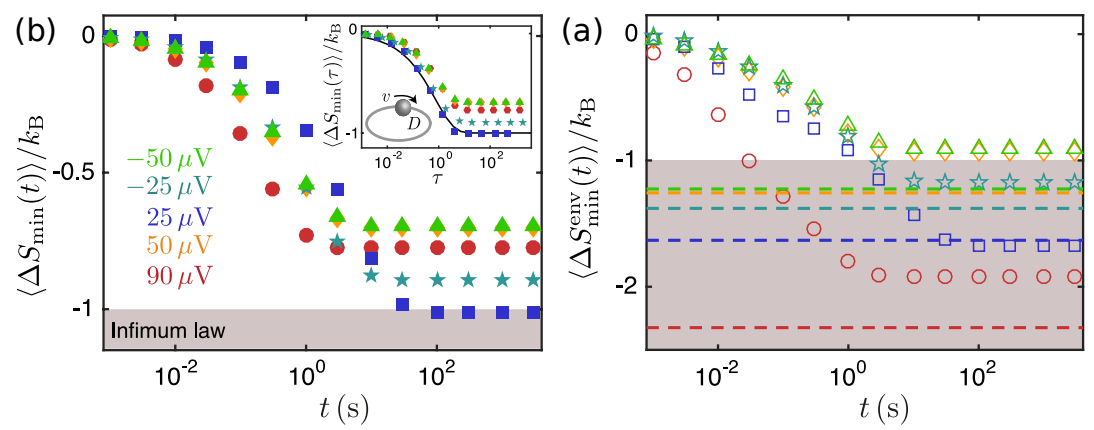

Figure 5.4. The experimental time dependence of the average of (a) the entropy production minimum $\left\langle\Delta S_{\min }(t)\right\rangle$ and (b) the minimum entropy exchange with environment $\left\langle\Delta S_{\min }^{\text {env }}(t)\right\rangle$ for different values of the bias voltage (shown in different colors) in the double dot. The shaded area marks the bound (5.18) and the dashed lines are the bound (5.19). The inset in (a) shows the variation of $\left\langle\Delta S_{\min }^{\mathrm{env}}(t)\right\rangle$ with a rescaled time $\tau=\langle\dot{S}\rangle t$. The black curve is $\left\langle\Delta S_{\min }(t)\right\rangle$ for a system with continuous $\Delta S(t)$, given by $-\operatorname{erf}(\sqrt{\tau} / 2)+(\tau / 2) \operatorname{erfc}(\sqrt{\tau} / 2)-\sqrt{\tau / \pi} \exp (-\tau / 4)$, see Ref. [38]. Adapted from Publication III.

using Eq. (5.19), we obtain the bound on maximum amount of heat absorbed by an isothermal mesoscopic system from its environment in a time interval $[0, t]$ as

$$
\left\langle Q_{\max }(t)\right\rangle \leq k_{\mathrm{B}} T\left[1+\sum_{n} p(n) \ln \frac{p(n)}{p_{\min }}\right] .
$$

This relation holds regardless of the system size or complexity. For systems in contact with nonequilibrium environment, the average maximum heat can be roughly estimated by replacing $T$ with an effective temperature $T_{\text {eff }}$ in Eq. (5.20). 
Stochastic thermodynamics of an electronic double dot 


\section{First-passage times and extreme fluctuations}

The first-passage time is defined as the time taken by a stochastic process, such as entropy production in Chapter 5 and the net charge transfered through a conductor in Sec. 2.4 (see Fig. 2.7), to reach a certain threshold for the first time. This passage time has been studied over centuries [8, 3944,58 ] in various contexts. However, the experimental results on the time needed for a certain amount of electric charge to be transfered through a conductor for the first time has not been presented before.

In Publication IV, we present the passage times for such events with the experimental data from the double dot setup, see Sec. 2.4. This chapter gives an overview of the theoretical background needed to understand these results. The chapter is organized as follows. We start with the expressions for the first-passage probability and extreme values for a general stochastic process. Next, we focus on the case of a tunnel junction which we implement to obtain the passage times for the net charge transferred in a more complicated structure, i.e., an electronic double dot system (Sec. 2.4). The chapter ends with a short discussion on the fluctuation relation between the first-passage times for positive and negative thresholds on the number of charge transfered through the double dot.

\subsection{Introduction}

Here we present a framework on how to calculate the statistics of the firstpassage times and extreme values associated with a stochastic process. For the sake of simplicity, we focus on a process of charge transfer, for example the net number of electrons transported through a double dot, see Fig. 2.7 (d). However, the method presented here can be used for any stochastic process.

We define $N(t)$ as the number of charges (charge carriers) transferred through a device in a given time $t$. The associated probability of $N$ electrons to tunnel through the device in time $t$ is defined as $P(t, N)$. 


\subsubsection{Charge distribution $P(t, N)$ : its evolution equation and properties}

The time evolution equation of $P(t, N)$ can, in general, be written as

$$
\frac{\partial P(t, N)}{\partial t}=[\mathcal{L} P](t, N),
$$

where $\mathcal{L}$ is a linear operator. We can define the cumulant generating function $\mathcal{F}(\chi)$ corresponding to the distribution $P(N, t)$ as

$$
e^{t \mathcal{F}(\chi)}=\sum_{N} e^{i \chi N} P(t, N)
$$

where $\chi$ is the counting field. The inverse transformation reads

$$
P(t, N)=\int_{-\pi}^{\pi} \frac{d \chi}{2 \pi} e^{-i \chi N} e^{t \mathcal{F}(\chi)} .
$$

This is the discretized version of Eq. (4.19). We assume that the distribution $P(t, N)$ satisfies the following fluctuation relation

$$
P(t, N)=e^{\gamma N} P(t,-N),
$$

where $\gamma=\mathrm{eV} /\left(k_{B} T\right)$ for $N$ as the net number of charges transferred through a device, subjected to bias voltage $V$ at temperature $T$ in time $t$. $P(t,-N)$ is defined as the probability of $N$ electrons to tunnel through the device in the direction opposite to bias in time $t$.

Using the distribution $P(t, N)$, we construct a new distribution

$$
P_{N_{0}}(t, N)=P(t, N)-e^{\gamma N_{0}} P\left(t, N-2 N_{0}\right) .
$$

This distribution is the difference of two solutions to Eq. (6.1), namely, $P_{1}(t, N)$ and $P_{2}(t, N)$ with initial conditions $P_{1}(0, N)=\delta_{N, 0}$ and $P_{2}(0, N)=$ $e^{\gamma N_{0}} \delta_{N, 2 N_{0}}$, respectively. Hence, $P_{N_{0}}(t, N)$ is also a solution to Eq. (6.1).

This distribution $P_{N_{0}}(t, N)$ has an interesting property of vanishing probability $P_{N_{0}}\left(t, N_{0}\right)=0$ for all $t$. This is known as an absorbing boundary. This property is a consequence of the fluctuation relation (6.4).

In Fig. 6.1, we illustrate sample trajectories corresponding to the charge transfer, $N(t)$, through the double dot sample in time $t$. The blue lines represent trajectories $N(t)$ which touch the boundary (a) $N_{0}=-9$ and (b) $N_{0}=9$ within the first 6 seconds. The light blue indicates the traces after they have been in contact with the boundary. The green lines are the traces which do not touch the boundary within the $6 \mathrm{~s}$ time window.

\subsubsection{Survival probability}

Using the distribution $P_{N_{0}}(t, N)$, we construct what we call survival probability $Q\left(t, N_{0}\right)$ of the trace to remain below (above) the positive (negative) 

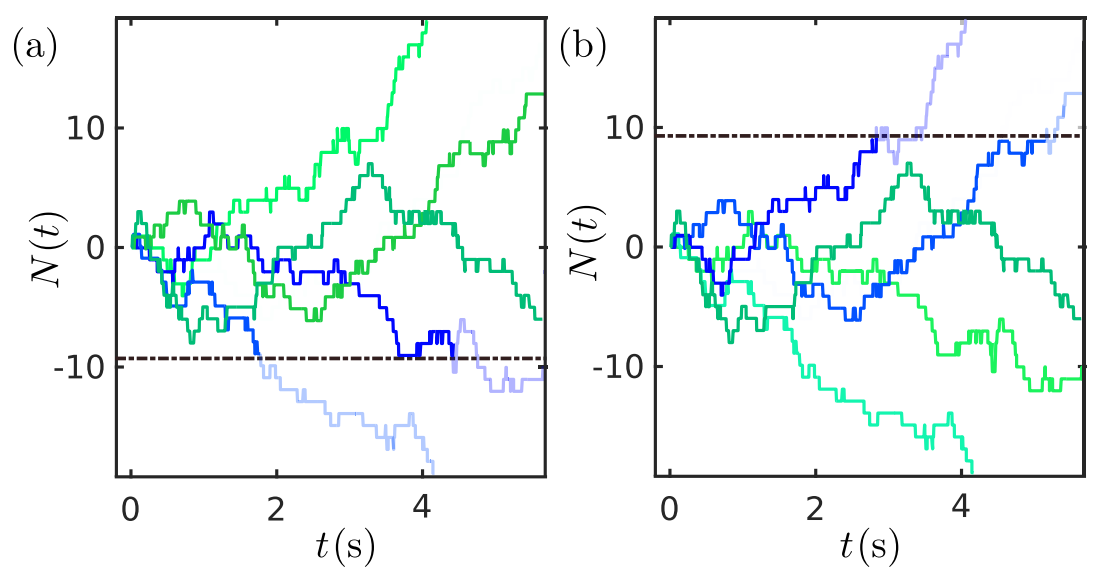

Figure 6.1. Experimental demonstration of survival probabilities for different absorbing boundaries $N_{0}$ on the time traces of net number of electrons transferred $N(t)$ through an electronic double dot (Sec. 2.4). The survival probability is calculated as the ratio of number of trajectories that do not touch or cross the boundary to the total number of traces under consideration. The green (blue) lines show the traces that survive (touch) the boundary with (a) $N_{0}=-9$ and (b) $N_{0}=9$ in time $t=6 \mathrm{~s}$.

threshold $N_{0}$ as

$$
Q\left(t, N_{0}\right)= \begin{cases}\sum_{N=-\infty}^{N_{0}-1} P_{N_{0}}(t, N) & \text { if } N_{0}>0 \\ \sum_{N=N_{0}+1}^{+\infty} P_{N_{0}}(t, N) & \text { if } N_{0}<0 .\end{cases}
$$

For positive $N_{0}$, the sum is over all the possible values of $N<N_{0}$ that the trace can attain before hitting the boundary $N=N_{0}$. As a consequence, the sum of $P_{N_{0}}(t, N)$ gives the probability of a trace $N(t)$ to survive the boundary $N=N_{0}$. In the experiments, the distribution $Q\left(t, N_{0}\right)$ is calculated as the ratio of number of traces that do not attain the value $N_{0}$ during time $t$ to the total number of traces under consideration.

In the case of negative $N_{0}$, the summation over $N>N_{0}$ results in the survival probability $Q\left(t, N_{0}\right)$ as the sum of $P_{N_{0}}(t, N)$ for all the traces before they attain the boundary. The summations in Eq. (6.6) hold for the traces where $N$ is integer, like in the case of net electron transfer through an electronic circuit (for example, see Fig. 6.1).

For the discrete traces $N_{\alpha}(t)$ with step size $\alpha \neq 1$ (for example in Sec. 6.2), one could rescale the trace $N_{\alpha}(t)=\alpha n(t)$. This results in the new modified trace $n(t)$ to be discrete with a step size 1 and the survival probability can be again defined by Eqs. (6.6) with $N$ replaced by $n$. In the case of continuous $N(t)$, the summations in Eq. (6.6) are replaced by integration, 
i.e.,

$$
Q\left(t, N_{0}\right)= \begin{cases}\int_{-\infty}^{N_{0}} d N P_{N_{0}}(t, N), & \text { if } N_{0}>0, \\ \int_{N_{0}}^{+\infty} d N P_{N_{0}}(t, N), & \text { if } N_{0}<0,\end{cases}
$$

where $P_{N_{0}}(t, N)$ now denotes a probability density function.

\subsubsection{First-passage-time distribution}

Let us consider the time steps to be discrete, with the $i$ th time step being represented by $t_{i}$. The difference $Q\left(t_{i-1}, N_{0}\right)-Q\left(t_{i}, N_{0}\right)$ will result in the probability of a trace $N(t)$ to touch the boundary $N_{0}$ for the first time at $t=t_{i}$. We call this time $t_{i}$ as the first-passage time. For the continuous time case, we define the distribution of first-passage times, $p_{N_{0}}(t)$, as the time derivative of survival probability $Q\left(t, N_{0}\right)$,

$$
p_{N_{0}}(t)=-\frac{\partial}{\partial t} Q\left(t, N_{0}\right) \text {. }
$$

First-passage-time distribution $p_{N_{0}}(t)$ (FPTD) gives the probability of a trace to cross or touch the boundary $N_{0}$ for the first time at $t$. The expression (6.8) is valid for both discrete and continuous $N_{0}$ because the partial derivate only demands continuity in time.

\subsubsection{Distribution of extreme-values}

Assuming that all traces start with $N=0$, the distribution $Q\left(t, N_{0}\right)$ is also the probability of a trajectory $N(t)$ to have an extreme value lying between 0 and $N_{0}$, due to the presence of absorbing boundary at $N_{0}$. For example, in Fig. 6.1 (a) the traces that survive the boundary (green lines) have a minimum value as $N_{0}^{-}=N_{0}+1=-8$ or higher.

Similarly, in panel (b), the traces with non-zero probabilities $P_{N_{0}}(t, N)$ (green lines) throughout the duration shown in the figure are the ones with a maximum value limited to 8 or lower. The maximum (minimum) values of the traces for positive (negative) boundaries are lower (upper) bounded by 0 , because all the traces follow the initial condition $N(t=0)=0$.

For positive threshold $N_{0}$, the survival probability $Q\left(t, N_{0}\right)$ is the ratio of number of traces which attain a maximum value of $N(t)<N_{0}$ to the total number of traces. In the case of discrete processes with the step size 1, $Q\left(t, N_{0}\right)$ gives the probability of traces to have a maximum value of $N_{0}-1$ or less. Similarly, $Q\left(t, N_{0}+1\right)$ gives the probability to have a maximum of $N_{0}$ or less. This results in the difference,

$$
p_{\max }\left(t, N_{0}\right)=-Q\left(t, N_{0}\right)+Q\left(t, N_{0}+1\right),
$$

as the probability of obtaining $N_{0}$ as the maximum number of charges transferred in a given time $t$. We call the distribution $p_{\max }\left(t, N_{0}\right)$ as the probability of obtaining $N_{0}$ as the maximum of a trace. 
(a)

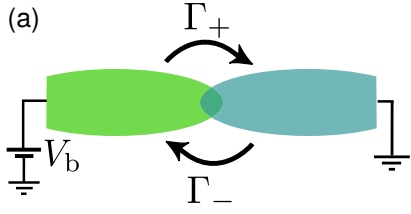

(b)

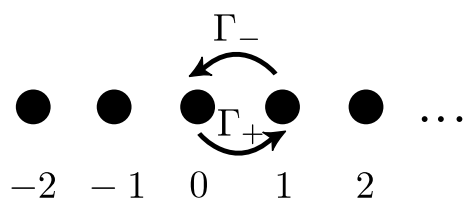

Figure 6.2. (a) A sketch of a tunnel junction subjected to bias voltage $V_{\mathrm{b}}$. An electron tunnels from the left lead (green) to the right lead (turquoise) and backwards with rates $\Gamma_{+}$and $\Gamma_{-}$respectively. (b) An equivalent model showing a particle jumping on a chain of sites, with forward and backward rates given by $\Gamma_{+}$and $\Gamma_{-}$respectively.

For $N_{0}<0$, the difference

$$
p_{\min }\left(t, N_{0}\right)=-Q\left(t, N_{0}\right)+Q\left(t, N_{0}-1\right)
$$

gives the probability of obtaining $N_{0}$ as the minimum number of charges transferred in a given time $t$. Thus, the distribution $p_{\min }\left(t, N_{0}\right)$ is defined as the probability of obtaining $N_{0}$ as the minimum for a given trace.

For a continuous $N(t)$, the differences in Eqs. (6.9) and (6.10) are replaced by the partial derivates with respect to $N$ at the boundary $N_{0}$, i.e.,

$$
\begin{array}{ll}
p_{\max }\left(t, N_{0}\right)=\left.\frac{\partial Q(t, N)}{\partial N}\right|_{N_{0}^{-}} & \text {if } N_{0}>0 \\
p_{\min }\left(t, N_{0}\right)=\left.\frac{\partial Q(t, N)}{\partial N}\right|_{N_{0}^{+}} & \text {if } N_{0}<0 .
\end{array}
$$

Here, $N_{0}^{-}$and $N_{0}^{+}$imply respectively the left and right derivative at the boundary $N=N_{0}$.

The formalism mentioned above is valid for a stochastic process of which the probability distribution follows the fluctuation relation (6.4) and the evolution equation can be written as Eq. (6.1). In the following sections, we present the calculation of first-passage times $p_{N_{0}}(t)$ for a tunnel junction and then use this model to approximate these distributions for the net number of charges transferred through a double dot system (see Sec. 2.4).

\subsection{Charge transfer through a tunnel junction}

Let us consider the simplest electronic setup, i.e., a tunnel junction (Sec. 2.4), which allows the transfer of particles with charge $e^{*}=\alpha e$. Here, $\alpha$ is the step size of the discrete stochastic trajectory, as mentioned in Sec. 6.1.2. We define $P\left(t, N^{*}\right)$ as the probability of $N^{*}$ such particles to tunnel through the junction in time $t$. The rates of particles to jump from left to right and vice versa are denoted as $\Gamma_{+}$and $\Gamma_{-}$, respectively. Figure 6.2 (a) shows the sketch of a tunnel junction with voltage $V_{\mathrm{b}}$ applied between the leads. Panel (b) shows a similar model for the hopping process on the chain of sites in a discrete lattice, i.e., an asymmetric random walk. The location of 
particle in latter is equivalent to the net number of electrons transferred in an electronic circuit, such as the tunnel junction in Fig. 6.2 (a).

The evolution equation of $P\left(t, N^{*}\right)$ for such a system is given by

$$
\frac{\partial P\left(t, N^{*}\right)}{\partial t}=\Gamma_{+} P\left(t, N^{*}-1\right)-\left(\Gamma_{-}+\Gamma_{+}\right) P\left(t, N^{*}\right)+\Gamma_{-} P\left(t, N^{*}+1\right),
$$

with the initial condition $P\left(0, N^{*}\right)=\delta_{N^{*}, 0}$. The cumulant generating function is [135]

$$
\mathcal{F}(\chi)=\Gamma_{+}\left(e^{i \chi}-1\right)+\Gamma_{-}\left(e^{-i \chi}-1\right),
$$

which describes a bidirectional Poissonian process. The solution to evolution equation (6.12) is well known and is given by

$$
P\left(t, N^{*}\right)=\int_{-\pi}^{\pi} \frac{d \chi}{2 \pi} e^{\mathcal{F}(\chi) t} e^{-i N^{*} \chi}=e^{-\Gamma_{\Sigma} t}\left(\frac{\Gamma_{+}}{\Gamma_{-}}\right)^{N^{*} / 2} I_{N^{*}}\left(2 \sqrt{\Gamma_{+} \Gamma_{-}} t\right),
$$

with $\Gamma_{\Sigma}=\Gamma_{+}+\Gamma_{-}$and $I_{\beta}(x)$ is the $\beta$ th order modified Bessel function of the first kind with argument $x$. Additionally, the probability distribution $P\left(t, N^{*}\right)$ satisfies the fluctuation theorem

$$
\frac{P\left(t, N^{*}\right)}{P\left(t,-N^{*}\right)}=\left(\frac{\Gamma_{+}}{\Gamma_{-}}\right)^{N^{*}} .
$$

Here, we have used the property of modified Bessel function, $I_{-\beta}(x)=I_{\beta}(x)$ if $\beta$ is an integer. Using the distribution $P\left(t, N^{*}\right)$ for particle transfer through a tunnel junction, Eq. (6.14), and the definition of passage times, Eq. (6.8), we obtain the FPTD

$$
p_{N_{0}}(t)=\frac{\left|N_{0}^{*}\right|}{t} I_{N_{0}^{*}}\left(2 \sqrt{\Gamma_{+} \Gamma_{-}} t\right)\left(\frac{\Gamma_{+}}{\Gamma_{-}}\right)^{N_{0}^{*} / 2} e^{-\Gamma_{\Sigma} t},
$$

where the effective boundary on particle transfer is

$$
N_{0}^{*}=\left\lfloor\frac{N_{0}}{\alpha}\right\rceil .
$$

The symbol $\lfloor a\rceil$ represents rounding the argument $a$ to nearest integer, such that the modified Bessel function $I_{\lfloor a\rceil}(x)$ has integer order $\lfloor a\rceil$.

In the experiments, we can measure the electron transfer through the double dot system in Fig. 2.4. Therefore, it is intuitive to define the absorption boundary in terms of net electron flow. We denote this boundary as $N_{0}$. When using coarse-grained theoretical models, such as the one in the next section, the net electron flow does not have to be exactly the same as the net particle flow. However, the boundary $N_{0}$ still imposes the condition $N_{0}^{*}$ on the net particle flow such that the total amount of charge transferred by the particles is the same as the one carried by electrons, i.e., $N_{0}^{*} e^{*}=N_{0} e$. In the following section, we use this simple model to approximate more complicated dynamics in an electronic double dot system. 
(a)
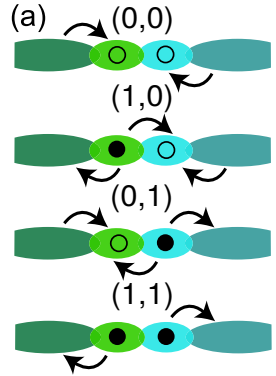

(b)

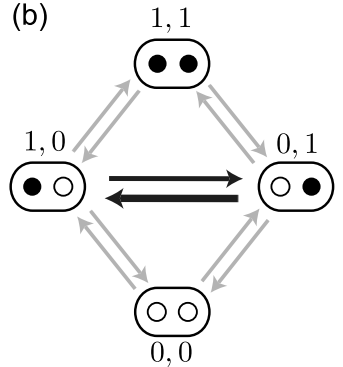

(c)

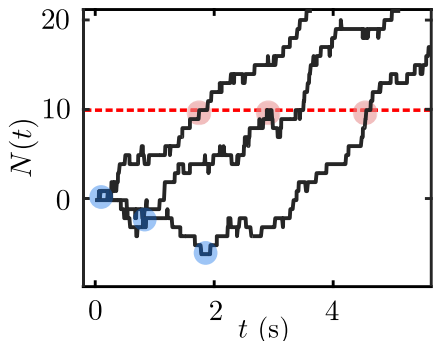

Figure 6.3. (a) The sketch of a double dot structure showing four probable configurations the system can acquire. The black filled (open) circle represents the presence (absence) of an extra electron on the island and black arrows are the possible transitions. (b) The sketch showing four probable states in a double dot, and the probable transitions between each state. For simplicity, we have removed the transitions $(0,0) \rightleftarrows(1,1)$, see Fig 2.5 , due to their negligible number, $<1 \%$, as compared to the most frequent ones. The electron transfer from $(0,1) \rightarrow(1,0)$ is favored in the system, when a positive bias voltage $\left(V_{\mathrm{b}}\right)$ is applied from the left lead. (c) The black lines are the three different realizations of the time traces of the net number of electrons transported from right to left island in the double dot with $V_{\mathrm{b}}=90 \mu \mathrm{V}$. The red circles mark the time it takes for these traces to touch the boundary $N_{0}=10$ for the first time. The blue circles illustrate the minimum number of electrons transferred from right to left during the given time interval, i.e., $6 \mathrm{~s}$. They can also be interpreted as maximum number of electrons transferred against the flow.

\subsection{Approximation for a double dot system}

In this section, we use the fore-mentioned tunnel junction model to calculate the distribution of first-passage times of electron transfer through an electronic double dot, and compare it with the distribution obtained in the experiments.

Figure 6.3 (a) and (b) show a schematic sketch of the sample with the four system states and the possible transitions from each state. The sample has three junctions and the net electron transfer through these three junctions may differ at most by two electrons at a given time due to charge conservation $^{1}$.

In this section, we focus on the net charge transfer through the middle junction of the double dot from right to left island. Figure 6.3 (c) shows examples of net electron transfer traces for device bias $90 \mu \mathrm{V}$. We define the probability of $N$ electrons to be transferred through the middle junction of the double dot in time $t$ as $P(t, N)$. We can write the time evolution equation of this probability similar to Eq. (6.12). In this 4-level system model, the fluctuation relation (6.4) does not hold ${ }^{2}$, see Fig. 6.5 (a). However, if we

${ }_{1}^{1}$ Starting from the system with no (one) extra electron in any (each) of the island the system may land up in a state with both islands filled with one (no) extra electron at given time $t$. This will result in one extra (less) electron flow from left and right lead to their corresponding island, leading to the time trace of net charge transferred through middle junction differ from the one between both the islands and the corresponding leads by 1 electron.

2 The slight deviation of experimental data points (Fig. 6.5 (a)) from the expected 
replace the 4-state model by a simpler tunnel junction model (Fig. 6.2), the fluctuation relation holds and the effects of non-middle junction transitions are captured in the third cumulant $C_{3}$ of $P(t, N)$ via the effective charge $\alpha e$ of the particle transferred through the middle junction. This assumption is valid when the cumulants of $P(t, N)$ satisfy the condition

$$
\frac{\mathcal{C}_{1}\left|\mathcal{C}_{1} \mathcal{C}_{4}-\mathcal{C}_{2} \mathcal{C}_{3}\right|}{12 \mathcal{C}_{2}^{3}}\left(\frac{N}{\mathcal{C}_{1} t}-1\right)^{2} \lesssim 1
$$

where the time $t$ is much larger than the system relaxation time ${ }^{3} \tau_{r}$ and the number of charges transferred $|N| \gg 1$. We have also assumed that $\mathcal{C}_{1}, \mathcal{C}_{3}>0$, and $\mathcal{C}_{2}^{2}>\mathcal{C}_{1} \mathcal{C}_{3}$. Once the above conditions are satisfied, we compute the effective rates and charge $\alpha$ as follows.

Using the cumulant generating function (6.13), we obtain the first four cumulants for the particle flow as

$$
\mathcal{C}_{1}^{*}=\left(\Gamma_{+}-\Gamma_{-}\right), \mathcal{C}_{2}^{*}=\left(\Gamma_{+}+\Gamma_{-}\right), \mathcal{C}_{3}^{*}=\left(\Gamma_{+}-\Gamma_{-}\right), \mathcal{C}_{4}^{*}=\left(\Gamma_{+}+\Gamma_{-}\right) .
$$

These cumulants, $\mathcal{C}_{j}^{*}$ are related to the experimentally obtained cumulants for the total number of electrons transferred $\left(\mathcal{C}_{j}\right)$ by a factor of the charge of particle ${ }^{4}$, i.e., $\mathcal{C}_{j}=\alpha^{j} \mathcal{C}_{j}^{*}$. These cumulants can be written as

$\mathcal{C}_{1}=\alpha\left(\Gamma_{+}-\Gamma_{-}\right), \mathcal{C}_{2}=\alpha^{2}\left(\Gamma_{+}+\Gamma_{-}\right), \mathcal{C}_{3}=\alpha^{3}\left(\Gamma_{+}-\Gamma_{-}\right), \mathcal{C}_{4}=\alpha^{4}\left(\Gamma_{+}+\Gamma_{-}\right)$.

Using $C_{i}$, we obtain the effective charge of the particle $\alpha$ and the rates as

$$
\alpha=\sqrt{\frac{\mathcal{C}_{3}}{\mathcal{C}_{1}}}, \Gamma_{+}=\frac{\mathcal{C}_{1}}{2 \mathcal{C}_{3}}\left(\mathcal{C}_{2}+\mathcal{C}_{1} \sqrt{\mathcal{C}_{3} / \mathcal{C}_{1}}\right), \Gamma_{-}=\frac{\mathcal{C}_{1}}{2 \mathcal{C}_{3}}\left(\mathcal{C}_{2}-\mathcal{C}_{1} \sqrt{\mathcal{C}_{3} / \mathcal{C}_{1}}\right) .
$$

The fourth cumulant $\mathcal{C}_{4}$ can be presented as a function of rates and $\alpha$ : $\mathcal{C}_{4}=\alpha^{4}\left(\Gamma_{+}+\Gamma_{-}\right)=\mathcal{C}_{3} \mathcal{C}_{2} / \mathcal{C}_{1}$. Similarly, in this approximation, all the higher order cumulants of distribution $P(t, N)$ can be presented in terms of the first three cumulants of the distribution.

Using the effective charge and rates [defined in Eqs. (6.21)] to the firstpassage-time distribution for tunnel junction model (6.16), we obtain the first-passage times for $N_{0}$ electrons to transfer through the double dot system as

$$
p_{N_{0}}(t)=\exp \left[-\frac{\mathcal{C}_{1} \mathcal{C}_{2}}{\mathcal{C}_{3}} t\right]\left(\frac{\mathcal{C}_{2}+\sqrt{\mathcal{C}_{1} \mathcal{C}_{3}}}{\mathcal{C}_{2}-\sqrt{\mathcal{C}_{1} \mathcal{C}_{3}}}\right)^{N_{0}^{*} / 2} \frac{\left|N_{0}^{*}\right|}{t} I_{N_{0}^{*}}\left(\frac{\mathcal{C}_{1} \sqrt{\mathcal{C}_{2}^{2}-\mathcal{C}_{1} \mathcal{C}_{3}}}{\mathcal{C}_{3}} t\right),
$$

black line is due to the limitations in validity of the detailed balance (2.31).

${ }^{3}$ In the experiments, we calculate the system relaxation time as the inverse of the eigenvalue of the transition rates matrix, which has the real part closest to zero among its non-zero eigenvalues. $\tau_{r}=8.7 \mathrm{~ms}$ for the double dot system at bias voltage $V_{\mathrm{b}}=90 \mu \mathrm{V}$.

${ }^{4}$ The charge transported by $N^{*}$ particles is same as the total amount of charge transferred by $N$ electrons, resulting in $N=\alpha N^{*}$. This implies $\left\langle N^{j}\right\rangle=\left\langle\left(\alpha N^{*}\right)^{j}\right\rangle$ and $\mathcal{C}_{j}=\alpha^{j} \mathcal{C}_{j}^{*}$. 

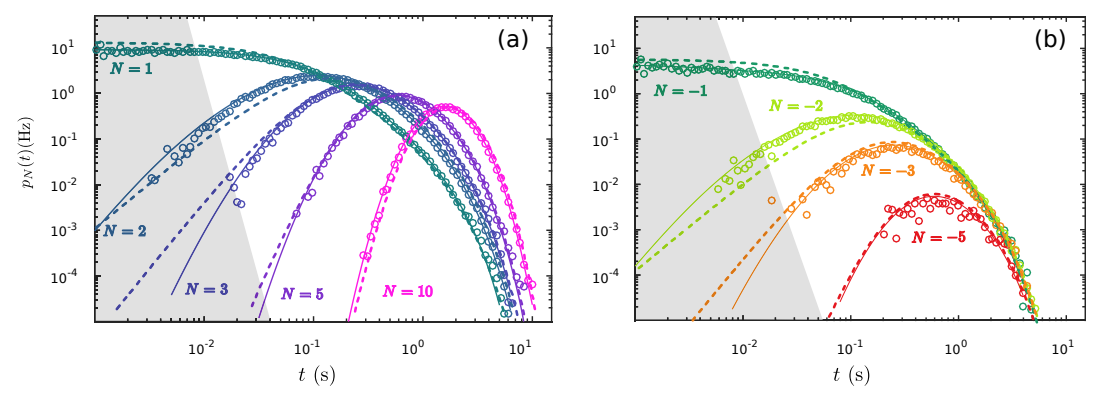

Figure 6.4. First-passage-time distributions for different threshold on net electron transfer $N$ for device bias $90 \mu \mathrm{V}$. The experimental data points (symbols), the exact theory [59] (solid lines), and the theory (6.22) (dashed lines). The shaded region shows the time regime where the theory (6.22) fails marked by condition (6.18). Adapted from Publication IV.

where the FPTD for $N_{0}$ electrons to tunnel through the middle junction $p_{N_{0}}$ is same as the FPTD for $N_{0}^{*}$ charged particle, with effective charge $\alpha$, to pass through the junction and the effective number of charge transferred $N_{0}^{*}=\left\lfloor N_{0} / \alpha\right\rceil$. In Fig. 6.4, we present the experimental FPTD (symbols) for different threshold values for double dot sample with bias $V_{\mathrm{b}}=90 \mu \mathrm{V}$. The theory (6.22) (dashed line) with cumulants calculated from experimental data is in agreement with the experiments when the condition (6.18) is satisfied. The shaded area marks the time for which the constraint Eq. (6.18) does not hold.

The cumulants $\mathcal{C}_{j}$ can be computed directly from the electron counting experiments using the probability distribution of the net number of electrons transferred in fixed time $t$ through the double dot system $P(t, N)$. It can also be calculated using the transition rates $\Gamma_{m \rightarrow n}$ for the system. Table 2.1 provides a list of transition rates between different states in the double dot at bias voltage ranging from $-50 \mu \mathrm{V}$ to $90 \mu \mathrm{V}$.

We compute the cumulants using both the above mentioned approach and obtain the first four cumulants as $\mathcal{C}_{1}=4.60 \mathrm{~Hz}, \mathcal{C}_{2}=9.27 \mathrm{~Hz}, \mathcal{C}_{3}=2.18$ $\mathrm{Hz}, \mathcal{C}_{4}=3.96 \mathrm{~Hz}$ with an error bar of $\pm 0.2 \mathrm{~Hz}$, which is compatible with the statistical uncertainty. The exact theory (solid line in Fig. 6.4) is obtained using the approach mentioned in Ref. [59]. For details, see the supplementary material of Publication IV.

Formally, the distribution (6.22) can also be obtained for a system without any constraints on the validity of the fluctuation relation. In this case, we assume that the system follows Markovian dynamics and exploit the relation [136]

$$
P(t, N)=\int_{0}^{t} d t^{\prime} p_{N}\left(t^{\prime}\right) P\left(t-t^{\prime}, 0\right)
$$

to compute the first-passage times $p_{N}(t)$ using the probability $P(t, N)$. 
Taking the Laplace transform, the equation (6.23) can be re-written as

$$
p_{N}(t)=\int_{c-i \infty}^{c+i \infty} \frac{d s}{2 \pi i} e^{s t} \frac{\int_{-\pi}^{\pi} \frac{d \chi}{2 \pi} \frac{e^{-i \chi N}}{s-\mathcal{F}(\chi)}}{\int_{-\pi}^{\pi} \frac{d \chi}{2 \pi} \frac{1}{s-\mathcal{F}(\chi)}},
$$

where $\mathcal{F}(\chi)$ is defined in Eq. (6.2). This is equivalent to the first-passage distribution defined in Eq. (6.8) but without any restriction on the probability distribution $P(t, N)$ to satisfy the fluctuation relation (6.4). For details on this approach, please refer to the supplemental material of Publication IV. Next, we briefly discuss the behavior of passage times $p_{N}(t)$ in different limiting cases on time and the distribution $P(t, N)$.

In short-time limit, the modified Bessel function can be written as $I_{n}(t) \sim t^{n} / n$ ! and $\exp (-t) \rightarrow 1$. This reduces the distribution (6.22) to

$$
p_{N_{0}}(t)=\left(\frac{\mathcal{C}_{2}+\sqrt{\mathcal{C}_{1} \mathcal{C}_{3}}}{\mathcal{C}_{2}-\sqrt{\mathcal{C}_{1} \mathcal{C}_{3}}}\right)^{N_{0}^{*} / 2} \frac{\left|N_{0}^{*}\right|}{t}\left(\frac{\mathcal{C}_{1} \sqrt{\mathcal{C}_{2}^{2}-\mathcal{C}_{1} \mathcal{C}_{3}}}{\mathcal{C}_{3}} t\right)^{N_{0}^{*}} \propto t^{N_{0}^{*}-1} .
$$

We observe a power-law dependence of the distribution on time $t$ in the short-time regime. In long-time limit, the expression (6.22) reduces to

$$
p_{N_{0}}(t)=\frac{a_{N_{0}}}{t^{3 / 2}} \exp (-\Gamma t) \text {. }
$$

This recovers the exponential decay of the FPTD [59] with the parameters $a_{N_{0}}{ }^{5}$ depending on the cumulants and threshold charge $N_{0}$, and the decay rate $\Gamma=\mathcal{C}_{1}^{2} /\left[\mathcal{C}_{2}+\sqrt{\mathcal{C}_{2}^{2}-\mathcal{C}_{1} \mathcal{C}_{3}}\right]$ depending only on the cumulants.

Additionally, we recover the well known passage time distribution of diffusive processes [41, 42], using the Eq. (6.22) and assuming the probability $P(t, N)$ to be a Gaussian distribution. In this case, we have only first two cumulants, $\mathcal{C}_{1}$ and $\mathcal{C}_{2}$ as non-zero and in the long-time limit, the distribution (6.22) simplifies to

$$
p_{N_{0}}(t)=\frac{|N|}{\sqrt{2 \pi t^{3} \mathcal{C}_{2}}} \exp \left[\frac{-\left(N-\mathcal{C}_{1} t\right)^{2}}{2 \mathcal{C}_{2} t}\right] .
$$

Here, we have used the asymptotic limit [137] of the modified Bessel function, i.e., $I_{n}(x) \simeq e^{x-n^{2} /(2 x)} / \sqrt{2 \pi x}$.

The FPTD (6.22) suggests a relation between the passage times for negative and positive threshold, namely

$$
\frac{p_{N_{0}}(t)}{p_{-N_{0}}(t)}=\left(\frac{\mathcal{C}_{2}+\sqrt{\mathcal{C}_{1} \mathcal{C}_{3}}}{\mathcal{C}_{2}-\sqrt{\mathcal{C}_{1} \mathcal{C}_{3}}}\right)^{\left\lfloor\sqrt{\frac{\mathcal{C}_{1}}{\mathcal{C}_{3}}} N_{0}\right\rceil}
$$

Figure 6.5 (b) shows the ratio $p_{N_{0}}(t) / p_{-N_{0}}(t)$ for experimental data, exact theory and right hand side of relation (6.28). The inset presents the ratio of total probability $A_{N} / A_{-N}$ integrated over time, defined as

5 The functional form of $a_{N}$ is $a_{N}=\left(\frac{\mathcal{C}_{2}+\sqrt{\mathcal{C}_{1} \mathcal{C}_{3}}}{\mathcal{C}_{2}-\sqrt{\mathcal{C}_{1} \mathcal{C}_{3}}}\right)^{N / 2} \frac{|N|}{\left[\mathcal{C}_{2}^{2}-\mathcal{C}_{1} \mathcal{C}_{3}\right]^{1 / 4} \sqrt{2 \pi \mathcal{C}_{1} / \mathcal{C}_{3}}}$. 

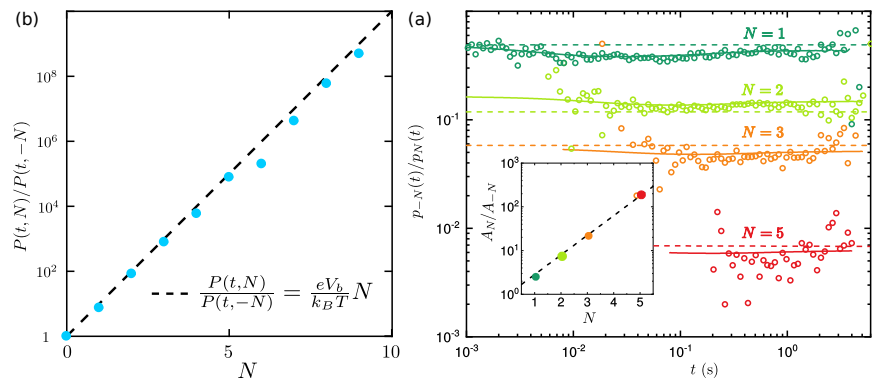

Figure 6.5. (a) The ratio of probability of charge distribution at a fixed time $t=1 \mathrm{~s}$ for double dot with bias $V_{\mathrm{b}}=90 \mu \mathrm{V}$. (b) The ratio of first-passage time probabilities corresponding to $-N$ and $N$ net charge transfer through the middle junction of double dot in time $t$, i.e., $p_{-N}(t) / p_{N}(t)$. The symbols are experimental data and the solid lines correspond to the ratio computed from exact theory [59]. The dashed line is the approximation (6.28). The inset shows the ratio of total probability integrated over time, $A_{N} / A_{-N}$. The symbols are experimental data and dashed line the approximation (6.28).

$A_{N}=\int_{0}^{\infty} d t p_{N}(t)$. The slope of $\ln \left[p_{N_{0}}(t) / p_{-N_{0}}(t)\right] \mathrm{v} / \mathrm{s} N_{0}$ curve is given by $\sqrt{\mathcal{C}_{1} / \mathcal{C}_{3}} \ln \left\{\left(\mathcal{C}_{2}+\sqrt{\mathcal{C}_{1} \mathcal{C}_{3}}\right) /\left(\mathcal{C}_{2}-\sqrt{\mathcal{C}_{1} \mathcal{C}_{3}}\right)\right\}$. This is equal to the bias voltage applied to the system normalized by thermal energy, i.e., $V_{b} /\left\{k_{B} T\right\}$ in thermal equilibrium. Similar fluctuation relation for the first-passage time on entropy produced by a system can be found in Ref. [38]. 
First-passage times and extreme fluctuations 


\section{Summary}

This thesis has focused on charge and entropy statistics in electronic systems. In the experiments, we used two different devices, a singleelectron transistor (SET) and an electronic double dot.

In Chapter 2, the basic physics of these devices were presented with the measurement setup used for the research. We started with an introduction to the physics of a tunnel junction. Then, we presented the energy considerations of electron transport in a single-electron box, an SET and an electronic double dot. The double dot structure was discussed in more detail. We showed that in the studied regime, it acts as a classical 4-level system with Markovian dynamics. We also presented the honeycomb structure in the stability diagram as expected from such system at zero bias voltage [97]. In Chapter 3, we presented the experimental details including the sample fabrication and characterization steps.

In Chapter 4, we studied the filtered output current from a single-electron transistor (SET). We proposed a method to infer the switching rates of a random telegraph noise based on an analytical expression for the distribution of the filtered current. The filtering function used to convolve with the telegraph noise mimics the behavior of a slow detector. This method of computing the transition rates should work even in the case of a slow detector. We validated the model by applying it to the telegraph signal generated by an SET, for which also the conventional threshold method can be used. We demonstrated the efficiency of the proposed method by applying it to an SET with a slow detector, for which the conventional threshold method cannot be used. A possible next step would be to extend the formalism to multi-level telegraph noise, so that it can be used for the telegraph signal from devices such as the electronic double dot.

In Chapter 4, we also presented the distribution of the time-averaged current. We showed from experimental data that the logarithm of the distribution has an elliptic form in the long averaging-time limit, as suggested by the large deviation principle. Additionally, we found a fluctuation relation for the departure of time-averaged current from its mean value in the long-time limit. These results are valid irrespective of the source of the 
bistable telegraph signal. Hence, the results can be used for detection and characterization of spurious two-level fluctuators coupled to the devices.

In Chapters 5 and 6 , we focused on the results obtained by measurements on an electronic double dot. The double dot allows us to measure the direction of electron flow [85, 98], unlike the single-island structures [13], and hence provides an excellent test bench for the results of steady-state thermodynamics.

In Chapter 5, we computed the total stochastic entropy production in the double dot system at nonequilibrium steady state (NESS) using the time trace of its state. Subsequently, we showed the validity of the infimum law in the double dot system at NESS. Additionally, we presented a bound on the entropy exchange with its environment, which depends on the heterogeneity of the steady state distribution of system states. We verified this bound with the experimental data. This theory can be further extended to a system at non-stationary state and even further to a system which follows quantum evolutions.

In Chapter 6, we focused on the experimental results on the first-passage times of electron transport through the middle junction of the double dot. The chapter presented the relevant theory developed in Publication IV and its comparison to the experimental data. Furthermore, we presented the experimental test of a fluctuation relation between the first-passage times for a positive and a negative threshold of the same magnitude.

As a future step, the double dot sample can be used to study the backaction in the electronic system. Additionally, with proper modification the device can be used as a test bench for Cooper-pair splitting [138-152].

Recently, the thermodynamic uncertainty relations [62, 153, 154] have been the subject of numerous theoretical studies. The double dot sample presented in this thesis could be used as a test bench for these inequalities. Additionally, the experimental study of these relations can be carried out under the conditions where one of the assumptions of the theory is violated. In this way, one can explore new regimes not covered by the existing theory. 


\section{Summary}




\section{References}

[1] De Moivre, A. Approximatio ad summam terminorum binomii $(a+b) n$ in seriem expansi Nov (1733). English translation: A. De Moivre, The Doctrine of Chances, 2nd ed. (London, England: H. Woodfall, 1738), 235-243.

[2] Laplace, P.-S. Théorie analytique des probabilités (1812).

[3] Lyapunov, A. M. Comptes Rendus Acad. Sci. 132, 814-815 (1901).

[4] Cam, L. L. Stat. Sci. 1(1), 78-91 02 (1986).

[5] Touchette, H. Phys. Rep. 478(1-3), 1-69 (2009).

[6] Reinhart, C. M. and Rogoff, K. S. American Economic Review 99(2), 466-72 May (2009).

[7] Aloui, R., Aïssa, M. S. B., and Nguyen, D. K. J. Bank. Finance 35(1), 130 141 (2011).

[8] Perelló, J., Gutiérrez-Roig, M., and Masoliver, J. Phys. Rev. E 84, 066110 Dec (2011).

[9] Gumbel, E. J. Statistics of extremes. Courier Corporation, (2012).

[10] Sharma, A. S., Bunde, A., Dimri, V., and Baker, D. Extreme Events and Natural Hazards: The Complexity Perspective. Wiley, New York, (2013).

[11] Castleman, A. W., Toennies, J., and Zinth, W., editors. Overview of Photon Counting Techniques, 11-25. Springer Berlin Heidelberg, Berlin, Heidelberg (2005).

[12] Natarajan, C. M., Tanner, M. G., and Hadfield, R. H. Supercond. Sci. Technol. 25(6), 063001 (2012).

[13] Pekola, J. P., Saira, O.-P., Maisi, V. F., Kemppinen, A., Möttönen, M., Pashkin, Y. A., and Averin, D. V. Rev. Mod. Phys. 85(4), 1421 (2013).

[14] Zwanzig, R. W. J. Chem. Phys. 22(8), 1420-1426 (1954).

[15] Yamada, T. and Kawasaki, K. Prog. Theor. Phys. 38(5), 1031-1051 (1967).

[16] Jarzynski, C. Phys. Rev. Lett. 78, 2690-2693 Apr (1997).

[17] Crooks, G. E. Phys. Rev. E 60, 2721-2726 Sep (1999).

[18] Bochkov, G. N. and Kuzovlev, I. E. Sov. Phys. JETP 45, 125 (1977).

[19] Ross, J., Hunt, K. L. C., and Hunt, P. M. J. Chem. Phys. 88(4), 2719-2729 (1988). 
[20] Evans, D. J. and Searles, D. J. Phys. Rev. E 50, 1645-1648 Aug (1994).

[21] Gallavotti, G. and Cohen, E. G. D. Phys. Rev. Lett. 74(14), 2694 (1995).

[22] Crooks, G. E. J. Stat. Phys. 90(5), 1481-1487 Mar (1998).

[23] Kurchan, J. J. Phys. A 31(16), 3719 (1998).

[24] Lebowitz, J. L. and Spohn, H. J. Stat. Phys. 95, 333 (1999).

[25] Crooks, G. E. Phys. Rev. E 61, 2361-2366 Mar (2000).

[26] Harris, R. J. and Schütz, G. M. J. Stat. Mech. 2007(07), P07020 (2007).

[27] Bustamante, C., Liphardt, J., and Ritort, F. Phys. Today 58, 43 (2005).

[28] Pekola, J. P. Nature Phys. 11, 118 (2015).

[29] Ciliberto, S. Phys. Rev. X 7, 21051 (2017).

[30] Gavrilov, M., Chétrite, R., and Bechhoefer, J. Proc. Natl. Acad. Sci. U.S.A. 114, 11097 (2017).

[31] Martínez, I. A., Roldán, É., Dinis, L., and Rica, R. A. Soft Matter 13, 22 (2017).

[32] Saira, O.-P., Yoon, Y., Tanttu, T., Möttönen, M., Averin, D. V., and Pekola, J. P. Phys. Rev. Lett. 109(18), 180601 (2012).

[33] Koski, J. V., Sagawa, T., Saira, O.-P., Yoon, Y., Kutvonen, A., Solinas, P., Möttönen, M., Ala-Nissila, T., and Pekola, J. P. Nat. Phys. 9, 644 (2013).

[34] Hofmann, A., Maisi, V. F., Rössler, C., Basset, J., Krähenmann, T., Märki, P., Ihn, T., Ensslin, K., Reichl, C., and Wegscheider, W. Phys. Rev. B 93, 035425 Jan (2016).

[35] Sekimoto, K. Prog. Theor. Phys. Supp. 130, 17-27 (1998).

[36] Sekimoto, K. Stochastic Energetics, volume 799 of Lecture Notes in Physics. Springer Berlin Heidelberg, Berlin, Heidelberg, (2010).

[37] Seifert, U. Rep. Prog. Phys. 75(12), 126001 (2012).

[38] Neri, I., Roldán, É., and Jülicher, F. Phys. Rev. X 7, 011019 Feb (2017).

[39] Redner, S. A Guide to First-Passage Processes. Cambridge University Press, Cambridge, UK, (2001).

[40] Metzler, R., Redner, S., and Oshanin, G. First-passage phenomena and their applications. World Scientific, (2014).

[41] Schrödinger, E. Phys. Z. 16, 289 (1915).

[42] Smoluchowski, M. V. Phys. Z. 16, 318 (1915).

[43] Artime, O., Khalil, N., Toral, R., and San Miguel, M. Phys. Rev. E 98, 042143 Oct (2018).

[44] Yang, X., Liu, C., Li, Y., Marchesoni, F., Hänggi, P., and Zhang, H. P. Proc. Natl. Acad. Sci. 114(36), 9564-9569 (2017).

[45] Szabo, A., Schulten, K., and Schulten, Z. J. Chem. Phys. 72, 4350 (1980).

[46] Zwanzig, R., Szabo, A., and Bagchi, B. Proc. Natl. Acad. Sci. USA 89, 20 (1992). 
[47] Galburt, E. A., Grill, S. W., Wiedmann, A., Lubkowska, L., Choy, J., Nogales, E., Kashlev, M., and Bustamante, C. Nature 446(7137), 820 (2007).

[48] Qian, H. and Xie, X. S. Phys. Rev. E 74, 010902 Jul (2006).

[49] Bauer, M. and Cornu, F. J. Stat. Phys. 155, 703 (2014).

[50] Benichou, O., Chevalier, C., Klafter, J., Meyer, B., and Voituriez, R. Nat. Chem. 2, 472 (2010).

[51] Chandrasekhar, S. Astrophys J. 97, 263 (1943).

[52] Majumdar, S. N. Curr. Sci. 89, 2076 (2005).

[53] Wald, A. Sequential analysis. Courier Corporation, (1973).

[54] Siggia, E. D. and Vergassola, M. Proc. Natl. Acad. Sci. 110(39), E3704E3712 (2013).

[55] Roldán, É., Neri, I., Dörpinghaus, M., Meyr, H., and Jülicher, F. Phys. Rev. Lett. 115, 250602 Dec (2015).

[56] Tejedor, V., Voituriez, R., and Bénichou, O. Phys. Rev. Lett. 108, 088103 Feb (2012).

[57] Mejía-Monasterio, C., Oshanin, G., and Schehr, G. J. Stat. Phys. 2011(06), P06022 (2011).

[58] Chicheportiche, R. and Bouchaud, J.-P. First-passage Phenomena and Their Applications. (2014).

[59] Saito, K. and Dhar, A. Europhys. Lett. 114(5), 50004 (2016).

[60] Ptaszyński, K. Phys. Rev. E 97, 012127 Jan (2018).

[61] Gingrich, T. R. and Horowitz, J. M. Phys. Rev. Lett. 119, 170601 Oct (2017).

[62] Garrahan, J. P. Phys. Rev. E 95, 032134 Mar (2017).

[63] Hänggi, P., Talkner, P., and Borkovec, M. Rev. Mod. Phys. 62, 251-341 Apr (1990).

[64] Devoret, M. H. and Grabert, H. Introduction to Single Charge Tunneling. (1992).

[65] Wasshuber, C. Computational Single-Electronics. Springer-Verlag Wien, 1 edition, (2001).

[66] Gorter, C. J. Physica 17, 777 (1951).

[67] Neugebauer, C. A. and Webb, M. B. J. Appl. Phys. 33(1), 74-82 (1962).

[68] Giaever, I. and Zeller, H. R. Phys. Rev. Lett. 20(26), 1504-1507 Jun (1968).

[69] Lambe, J. and Jaklevic, R. C. Phys. Rev. Lett. 22(25), 1371-1375 Jun (1969).

[70] Kulik, I. O. and Shekhter, R. I. Zh. Eksp. Teor. Fiz. 68 (1975). [Sov. Phys. JETP 41, 308 (1975)].

[71] Averin, D., Zorin, A., and Likharev, K. Sov. Phys. JETP 61(2), 407-413 (1985).

[72] Dolan, G. J. and Dunsmuir, J. H. Physica B: Condensed Matter 152(1-2), 7-13 (1988). 
[73] Dolan, G. J. Appl. Phys. Lett. 31(5), 337 (1977).

[74] Fulton, T. A. and Dolan, G. J. Phys. Rev. Lett. 59(1), 109-112 Jul (1987).

[75] Pobell, F. Matter and Methods at Low Temperatures. Springer Berlin Heidelberg, Berlin, Heidelberg, (2007).

[76] Bardeen, J., Cooper, L. N., and Schrieffer, J. R. Phys. Rev. 106, 162-164 Apr (1957).

[77] Bardeen, J., Cooper, L. N., and Schrieffer, J. R. Phys. Rev. 108, 1175-1204 Dec (1957).

[78] Ashcroft, N. Solid State Physics. Philadelphia, (1976).

[79] Cho, I.-T., Lee, J.-M., Lee, J.-H., and Kwon, H.-I. Semiconductor Science and Technology 24(1), 015013 (2008).

[80] Court, N. A., Ferguson, A. J., Lutchyn, R., and Clark, R. G. Phys. Rev. B 77, 100501 Mar (2008).

[81] de Visser, P. J., Baselmans, J. J. A., Diener, P., Yates, S. J. C., Endo, A., and Klapwijk, T. M. Phys. Rev. Lett. 106, 167004 Apr (2011).

[82] Wilson, C. M. and Prober, D. E. Phys. Rev. B 69, 094524 Mar (2004).

[83] Lafarge, P., Pothier, H., Williams, E. R., Esteve, D., Urbina, C., and Devoret, M. H. Z. Phys. B 85(3), 327-332 Oct (1991).

[84] Lu, W., Ji, Z., Pfeiffer, L., West, K. W., and Rimberg, A. J. Nature 423, 422 EP - May (2003).

[85] Fujisawa, T., Hayashi, T., Tomita, R., and Hirayama, Y. Science 312(5780), 1634-1636 (2006).

[86] Gustavsson, S., Leturcq, R., Simovič, B., Schleser, R., Ihn, T., Studerus, P., Ensslin, K., Driscoll, D. C., and Gossard, A. C. Phys. Rev. Lett. 96, 076605 Feb (2006).

[87] Sukhorukov, E. V., Jordan, A. N., Gustavsson, S., Leturcq, R., Ihn, T., and Ensslin, K. Nat. Phys. 3, 243 (2007).

[88] Fricke, C., Hohls, F., Wegscheider, W., and Haug, R. J. Phys. Rev. B 76(15), 155307 (2007).

[89] Gustavsson, S., Leturcq, R., Studer, M., Shorubalko, I., Ihn, T., Ensslin, K., Driscoll, D. C., and Gossard, A. C. Surf. Sci. Rep. 64, 191 (2009).

[90] Flindt, C., Fricke, C., Hohls, F., Novotný, T., Netočný, K., Brandes, T., and Haug, R. J. Proc. Natl. Acad. Sci. USA 106(25), 10116-10119 Jun (2009).

[91] Ubbelohde, N., Fricke, C., Flindt, C., Hohls, F., and Haug, R. J. Nat. Commun. 3, 612 (2012).

[92] House, M. G., Xiao, M., Guo, G., Li, H., Cao, G., Rosenthal, M. M., and Jiang, H. Phys. Rev. Lett. 111(12), 126803 (2013).

[93] Maisi, V. F., Kambly, D., Flindt, C., and Pekola, J. P. Phys. Rev. Lett. 112(3), 36801 (2014).

[94] Likharev, K. K. and Zorin, A. B. Journal of Low Temperature Physics 59(3), 347-382 May (1985).

[95] Averin, D. V. and Likharev, K. K. Journal of Low Temperature Physics 62(3), 345-373 Feb (1986). 
[96] Breuer, H. P. and Petruccione, F. The Theory of Open Quantum Systems. Oxford University Press, USA, (2002).

[97] van der Wiel, W. G., De Franceschi, S., Elzerman, J. M., Fujisawa, T., Tarucha, S., and Kouwenhoven, L. P. Rev. Mod. Phys. 75(1), 1 (2002).

[98] Küng, B., Rössler, C., Beck, M., Marthaler, M., Golubev, D. S., Utsumi, Y., Ihn, T., and Ensslin, K. Phys. Rev. X 2, 011001 Jan (2012).

[99] Dutta, P. and Horn, P. M. Rev. Mod. Phys. 53, 497-516 Jul (1981).

[100] Kirton, M. and Uren, M. Advances in Physics 38(4), 367-468 (1989).

[101] Pekola, J. P., Maisi, V. F., Kafanov, S., Chekurov, N., Kemppinen, A., Pashkin, Y. A., Saira, O.-P., Möttönen, M., and Tsai, J. S. Phys. Rev. Lett. 105(2), 26803 (2010).

[102] Gloos, K., Koppinen, P. J., and Pekola, J. P. J. Phys. Condens. Matter 15(10), 1733 (2003).

[103] Pekola, J. and Kauppinen, J. Cryogenics 34(10), 843 - 845 (1994).

[104] Kemppinen, A., Meschke, M., Möttönen, M., Averin, D. V., and Pekola, J. P. Eur. Phys. J. Spec. Top. 172, 311 (2009).

[105] Zorin, A. B. Rev. Sci. Instr. 66(8), 4296-4300 (1995).

[106] Jordan, A. N. and Sukhorukov, E. V. Phys. Rev. Lett. 93(26), 260604 Dec (2004).

[107] Dresselhaus, P. D., Ji, L., Han, S., Lukens, J. E., and Likharev, K. K. Phys. Rev. Lett. 72(20), 3226-3229 may (1994).

[108] Kogan, S. Phys. Rev. Lett. 81(14), 2986-2989 oct (1998).

[109] Wacker, A. and Schöll, E. J. Appl. Phys. 78(12), 7352-7357 (1995).

[110] Wernsdorfer, W., Orozco, E. B., Hasselbach, K., Benoit, A., Barbara, B., Demoncy, N., Loiseau, A., Pascard, H., and Mailly, D. Phys. Rev. Lett. 78(9), 1791-1794 mar (1997).

[111] Ingvarsson, S., Xiao, G., Parkin, S. S. P., Gallagher, W. J., Grinstein, G., and Koch, R. H. Phys. Rev. Lett. 85(15), 3289-3292 oct (2000).

[112] Urazhdin, S., Birge, N. O., Pratt, W. P., and Bass, J. Phys. Rev. Lett. 91(14), 146803 Oct (2003).

[113] Bezrukov, S. M. and Kasianowicz, J. J. Phys. Rev. Lett. 70(15), 2352 (1993).

[114] Verley, G., Van den Broeck, C., and Esposito, M. Phys. Rev. E 88, 032137 Sep (2013).

[115] Naaman, O. and Aumentado, J. Phys. Rev. B 73(17), 172504 May (2006).

[116] Utsumi, Y. Phys. Rev. B 75(3), 35333 (2007).

[117] Bagrets, D. A. and Nazarov, Y. V. Phys. Rev. B 67, 085316 Feb (2003).

[118] Bergli, J., Galperin, Y. M., and Altshuler, B. L. New J. Phys. 11, 25002 (2009).

[119] Lambert, N., Nori, F., and Flindt, C. Phys. Rev. Lett. 115(21), 216803 (2015).

[120] Gallavotti, G. and Cohen, E. G. D. J. Stat. Phys. 80(5), 931 (1995). 
[121] Seifert, U. Phys. Rev. Lett. 95(4), 40602 (2005).

[122] Private communication with Samu Suomela.

[123] Brémaud, P. An Introduction to Probabilistic Modeling. Undergraduate Texts in Mathematics. Springer New York, New York, NY, (1988).

[124] Jarzynski, C. Phys. Rev. E 56, 5018-5035 Nov (1997).

[125] Jarzynski, C. Annual Review of Condensed Matter Physics 2(1), 329-351 (2011).

[126] Gross, D. arXiv:cond-mat/0508721 (2005).

[127] Murashita, Y., Funo, K., and Ueda, M. Phys. Rev. E 90, 042110 Oct (2014).

[128] Esposito, M., Harbola, U., and Mukamel, S. Rev. Mod. Phys. 81(4), 1665 (2009).

[129] Ville, J. Etude critique de la notion de collectif. PhD thesis, Gauthier-Villars, Paris, (1939).

[130] Doob, J. L. Stochastic processes. Wiley, New York, (1953).

[131] Williams, D. Probability with martingales. Cambridge University Press, Cambridge, (1991).

[132] Liptser, R. S. and Shiryaev, A. N. Statistics of random processes: I \& II. Springer Science \& Business Media, Berlin, (2013).

[133] Chétrite, R., Gupta, S., Neri, I., and Roldán, É. Europhys. Lett. 124(6), 60006 (2019).

[134] Pigolotti, S., Neri, I., Roldán, É., and Jülicher, F. Phys. Rev. Lett. 119(14), 140604 (2017).

[135] Utsumi, Y., Golubev, D. S., and Schön, G. Phys. Rev. Lett. 96, 086803 Mar (2006).

[136] Siegert, A. J. F. Phys. Rev. 81, 617-623 Feb (1951).

[137] Dingle, R. B. and Dingle, R. Asymptotic expansions: their derivation and interpretation, volume 48. Academic Press London, (1973).

[138] Lesovik, G., Martin, T., and Blatter, G. Eur. Phys. J. B 24(3), 287-290 Dec (2001).

[139] Recher, P., Sukhorukov, E. V., and Loss, D. Phys. Rev. B 63, 165314 Apr (2001).

[140] Beckmann, D., Weber, H. B., and v. Löhneysen, H. Phys. Rev. Lett. 93, 197003 Nov (2004).

[141] Russo, S., Kroug, M., Klapwijk, T. M., and Morpurgo, A. F. Phys. Rev. Lett. 95, 027002 Jul (2005).

[142] Cadden-Zimansky, P., Wei, J., and Chandrasekhar, V. Nature Physics 5, 393 EP - Apr (2009).

[143] Hofstetter, L., Csonka, S., Nygård, J., and Schönenberger, C. Nature 461, $960 \mathrm{EP}$ - Oct (2009).

[144] Herrmann, L. G., Portier, F., Roche, P., Yeyati, A. L., Kontos, T., and Strunk, C. Phys. Rev. Lett. 104, 026801 Jan (2010). 
[145] Wei, J. and Chandrasekhar, V. Nat. Phys. 6, 494 EP - May (2010).

[146] Hofstetter, L., Csonka, S., Baumgartner, A., Fülöp, G., d'Hollosy, S., Nygård, J., and Schönenberger, C. Phys. Rev. Lett. 107, 136801 Sep (2011).

[147] Schindele, J., Baumgartner, A., and Schönenberger, C. Phys. Rev. Lett. 109, 157002 Oct (2012).

[148] Das, A., Ronen, Y., Heiblum, M., Mahalu, D., Kretinin, A. V., and Shtrikman, H. Nat. Commun. 3, 1165 EP - Nov (2012).

[149] Tan, Z. B., Cox, D., Nieminen, T., Lähteenmäki, P., Golubev, D., Lesovik, G. B., and Hakonen, P. J. Phys. Rev. Lett. 114, 096602 Mar (2015).

[150] Deacon, R. S., Oiwa, A., Sailer, J., Baba, S., Kanai, Y., Shibata, K., Hirakawa, K., and Tarucha, S. Nat. Commun. 6, 7446 EP - Jul (2015).

[151] Michałek, G., Domański, T., and Wysokiński, K. I. J. Supercond. \& Novel Magn. 30(1), 135-138 Jan (2017).

[152] Wrześniewski, K., Trocha, P., and Weymann, I. J. Phys.: Condens. Matter 29, 195302 (2017).

[153] Barato, A. C. and Seifert, U. Phys. Rev. Lett. 114(15) (2015).

[154] Horowitz, J. M. and Gingrich, T. R. Phys. Rev. E 96(2) (2017). 
The improbable is bound to happen one day.

-- E. J. Gumbel (Statistics of Extremes)

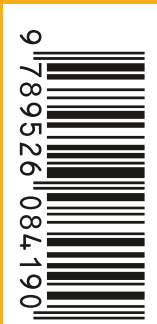

ISBN 978-952-60-8419-0 (printed)

ISBN 978-952-60-8420-6 (pdf)

ISSN 1799-4934 (printed)

ISSN 1799-4942 (pdf)

Aalto University

School of Science

Department of Applied Physics

www.aalto.fi

\author{
BUSINESS + \\ ECONOMY \\ ART + \\ DESIGN + \\ ARCHITECTURE \\ SCIENCE + \\ TECHNOLOGY \\ CROSSOVER \\ DOCTORAL \\ DISSERTATIONS
}

